\title{
Online Source Rate Control for Adaptive Video Streaming Over HSPA and LTE-Style Variable Bit Rate Downlink Channels
}

\author{
4 Jian Yang, Member, IEEE, Yongyi Ran, Shuangwu Chen, Weiping Li, Fellow, IEEE, and Lajos Hanzo, Fellow, IEEE
}

5 Abstract-Online source rate control (RC) is designed for video 6 streaming over high-speed packet access (HSPA) and Long-Term 7 Evolution (LTE)-style variable bit rate (VBR) downlink channels. 8 The problem is formulated as the adaptive adjustment of the 9 operational mode of a video encoder based on the buffer overflow 10 probability (BOP) feedback received from the radio link control 11 (RLC) layer at the base station (BS). This allows us to maximize 12 the attainable visual quality while keeping the transmitter BOP 13 below a desired threshold and maintaining a video delay as low as 14 possible. We derive an online measurement-based BOP estimation 15 model for the RLC buffer, which is capable of operating with 16 no prior knowledge of the channel variations and of the video 17 characteristics. Based on this estimation model, an online adaptive $18 \mathbf{R C}$ algorithm is proposed to seamlessly adapt the bit stream to 19 the characteristics of VBR channels. Our experiments are con20 ducted in multiuser scenarios using VBR video encoding combined 21 with adaptive modulation and coding (AMC) in the transceiver. 22 The results demonstrate that the proposed source $\mathrm{RC}$ regime 23 supports near-instantaneous yet smooth bit stream adaptability, 24 which makes it useful for HSPA and LTE-style systems for the 25 sake of accommodating unknown video traffic characteristics and 26 dynamically fluctuating propagation conditions.

28 Index Terms-Large deviation principle (LDP), rate control (RC), variable bit rate (VBR) channel.

\section{INTRODUCTION}

30 DVANCED transceiver techniques, including adaptive 32 modulation and coding (AMC) and hybrid automatic re33 peat request (HARQ), are widely applied in wireless systems to 34 combat the effects of both fading and interference [1], [2]. For 35 instance, both the third-generation $(3 \mathrm{G})$ and high-speed packet

Manuscript received January 10, 2014; revised July 21, 2014 and November 3, 2014; accepted January 24, 2015. This work was supported in part by the National Natural Science Foundation of China under Grant 61174062; by the State Key Program of the National Natural Science Foundation of China under Grant 61233003; and by the Fundamental Research Funds for the Central Universities. The work of L. Hanzo was supported by the European Research Council under an Advanced Fellow Grant. The review of this paper was coordinated by Prof. N. Arumugam.

J. Yang, Y. Ran, S. Chen, and W. Li are with the School of Information Science and Technology, University of Science and Technology of China, Hefei 230026, China (e-mail: jianyang@ ustc.edu.cn; yyran@ mail.ustc.edu.cn; chensw@mail.ustc.edu.cn; wpli@ustc.edu.cn).

L. Hanzo is with the School of Electronics and Computer Science, University of Southampton, Southampton SO17 1BJ, U.K. (e-mail: 1h@ecs.soton.ac.uk).

Color versions of one or more of the figures in this paper are available online at http://ieeexplore.iee.org.

Digital Object Identifier 10.1109/TVT.2015.2398515 access (HSPA) apply AMC and HARQ in the physical layer 36 relying on the channel quality indicator's (CQI) feedback from 37 the user equipment (UE) to achieve prompt link adaptation. The 38 Long-Term Evolution (LTE) Advanced and WiMax standards 39 also provide similar link adaptation based on AMC and short 40 time-slot duration periods. Naturally, agile link adaptation may 41 result in a time-varying effective throughput of the channel. 42 Therefore, supporting near-instantaneous bit rate adaptivity 43 is one of the most important features of video streaming 44 applications.

Rate control (RC) aims to control the video bit rate to match 46 the channel's achievable bit rate, given the prevalent channel 47 quality to keep the video quality as high as possible [3]. Most 48 existing studies of RC focus on allocating a bit rate budget to 49 each group of pictures (GOP), frames, or macroblocks at the 50 encoder. For instance, an efficient RC scheme was designed 51 for MPEG-4 based on a quadratic rate-distortion (R-D) model 52 invoked to maintain the target bit rate in [4]. In [5], the quadratic 53 $\mathrm{R}-\mathrm{D}$ model was also adopted to derive an adaptive $\mathrm{RC}$ for 54 H.264 to meet the target bit rate. In [6], RC was designed 55 for scalable H.264/Advanced Video Coding (AVC) encoding. 56 H.264 reference software JM [7] has implemented the JVT- 57 G012 [8] RC algorithm, which relies on a combination of the 58 available channel bandwidth, the frame rate, the target buffer 59 level, and the actual buffer fullness for determining the number 60 of bits allocated for the current frame. A range of AMC-based 61 schemes were conceived in [9] where no extra buffering was 62 used by the RC. The design philosophy was to instruct the 63 video encoder to produce the exact number of bits for each 64 video frame, which was affordable for the near-instantaneous 65 HSPA-style AMC transceiver mode that was periodically sig- 66 naled back from the receiver to the transmitter. However, the 67 aforementioned RC schemes rely on the knowledge of the target 68 bit rate, which again requires the feedback of the expected bit 69 rate based on the estimated channel quality. Unfortunately, the 70 channel's bit rate fluctuations are not known a priori at the 71 transmitter and the video encoder. Therefore, these RC schemes 72 may impose substantial video quality fluctuations.

Recently, a control-theoretic approach-based RC regime has 74 been proposed in [10] by jointly considering the encoder's RC 75 and network congestion control. More specifically, an empirical 76 R-D source model, a channel-induced distortion model, and 77 their linearized models were applied to formulate a mathe- 78 matically tractable system model. However, it is a challenge 79 to formulate accurate models when streaming videos over 80 
81 time-varying channels. Typically, intelligent packet scheduling 82 is used at the base station (BS) by most of the existing solutions 83 to achieve channel-quality-dependent adaptive video streaming 84 over wireless networks. Cross-layer-adaptive scalable video 85 streaming has been proposed by informing the media access 86 control (MAC) layer of the amount of payload and the index of 87 the modulation-and-encoding scheme to improve the attainable 88 video quality [11]. An adaptive scalable video streaming strat89 egy relaying on controlling the MAC buffer was presented in 90 [12]. Both the packet deadlines and the channel characteristics 91 were considered in video packet scheduling at the BS [13]. 92 The cross-layer design-aided transmission of scalable video 93 streams [14] involves packet scheduling combined with a video 94 frame dropping strategy at the BS. Most of these papers discuss 95 the channel-dependent adaptation of the MAC layer of the BS 96 where no signaling is fed back to instruct the video source to 97 adjust its bit rate. Dropping packets at the BS is a waste of 98 resources both in the backhaul router and the core network. It 99 was shown in [15] that packet dropping at the video source is 100 more efficient than MAC layer dropping at the BS when we 101 have to reduce the congestion in both the wired core network 102 and in the wireless medium to the UE. By translating the $103 \mathrm{CQI}$ values to the number of enhancement layers scheduled 104 for transmission, the video server facilitates adaptive video 105 streaming [16]. However, the link throughput at the BS is 106 determined not only by the CQI value but also by the MAC 107 layer scheduling strategy of a multiuser scenario. Moreover, 108 the bit rate of video clips having different amounts of motion 109 activity may be very different even if they have the same num110 ber of enhancement layers [17]. Therefore, such CQI-mapping111 based video source adaptation lacks robustness against the 112 time-variant number of users and against the heterogeneous 113 video bit rates. In [18], although a Markov decision process is 114 invoked for dynamic video scheduling, its offline computation 115 requires a priori knowledge of the channel dynamics. Hence, 116 a heuristic online scheduling algorithm is derived based on the 117 average channel throughput estimated with the aid of a first118 order autoregressive model [AR(1)]. In the HSPA/LTE system, 119 using a CQI-based link adaptation mechanism may lead to 120 a burst-by-burst transmission block adaptation, and the linear 121 estimator based AR(1) may not be appropriate for estimating 122 the average channel throughput.

123 Against this background, we proposed a novel online source 124 RC framework, where a buffer overflow probability (BOP) 125 estimator is employed at the radio link control (RLC) layer 126 of the BS, and the estimated BOP is signaled back to the 127 video source to control its bit rate. The motivation of using a 128 BOP-based feedback is that the BOP metric characterizes the 129 degree of matching between the video bit rate of the source 130 and the link throughput. Since the BOP is estimated before 131 a buffer overflow is encountered, its feedback may assist the 132 video source in promptly controlling the bit rate. In contrast to 133 the methods found in the open literature [9]-[14], [16], [18], the 134 main contributions of this paper are threefold.

- To enable the video encoder to generate a video stream that may be reliably delivered over variable bit rate (VBR) downlink channels, we formulate a constrained optimization problem subject to a constraint imposed on 138 the transmission BOP to guarantee a low delay.

- A BOP estimation model based on large deviation prin- 140 ciples (LDPs) [19] is proposed by monitoring the buffer 141 fullness and its variation at the BS's RLC layer. The 142 reason for applying the LDPs is because it accurately 143 characterizes the probability of rare events, which assists 144 us in achieving fine adjustment of the video source rate. 145

- We conceive an iterative RC algorithm to approach the 146 most beneficial encoder rate, thus circumventing the dif- 147 ficulty of directly solving the related constrained opti- 148 mization problem. The proposed RC algorithm allows the 149 encoder to adjust its bit rate to that of the VBR channel 150 without any a priori knowledge of both the channel 151 quality variations and of the characteristics of the video 152 source.

The remainder of this paper is organized as follows. 154 Section II describes the system model, including the formu- 155 lation of the source RC problem of video streaming over a 156 VBR downlink channel. In Section III, we apply the LDP in 157 [19] to derive a BOP estimation model, whereas an online 158 measurement-based RC is proposed in Section IV. Our numeri- 159 cal simulation results are presented in Section V to characterize 160 the attainable performance of the proposed algorithm. Finally, 161 our conclusions are offered in Section VI.

\section{System Model}

\section{A. System Overview}

Fig. 1 shows a typical video streaming scenario over a 166 wireless network. The main associated protocol stacks are also 167 shown in the lower part of the figure. Naturally, a video stream- 168 ing service involves not only maintaining the wireless connec- 169 tion between the mobile station (MS) and the base transceiver 170 station (BTS) but supporting the public network connection 171 (Internet) as well. The wired network provides high capacity 172 and stability; hence, video streaming over the wired network 173 has become a well-established service and has many successful 174 applications, including video conferencing, surveillance sys- 175 tems, and Internet Protocol (IP) television. By contrast, video 176 streaming over wireless networks faces unique challenges due 177 to the time-varying nature of the wireless channel and owing to 178 the scarcity of the system resources, which makes it difficult to 179 guarantee any specific video quality of service (QoS). Hence, 180 the wireless transmission in the video streaming service is likely 181 to be a bottleneck, which is the focus of this paper.

Fig. 2 shows the basic video processing in the video network 183 abstract layer (NAL) units of the 3GPP framework. A NAL 184 unit may be encapsulated in a Real-Time Transport Protocol 185 (RTP) data unit, and then, it may be transmitted over User 186 Datagram Protocol (UDP)/IP. HTTP-based streaming protocols 187 such as Apple HTTP Live Streaming (HLS) [20] are alternative 188 media streaming communication protocols, which are capable 189 of traversing any firewall or proxy server that lets through 190 standard HTTP traffic, unlike UDP-based protocols such as 191 RTP. 3GPP standardized an adaptive HTTP streaming protocol 192 


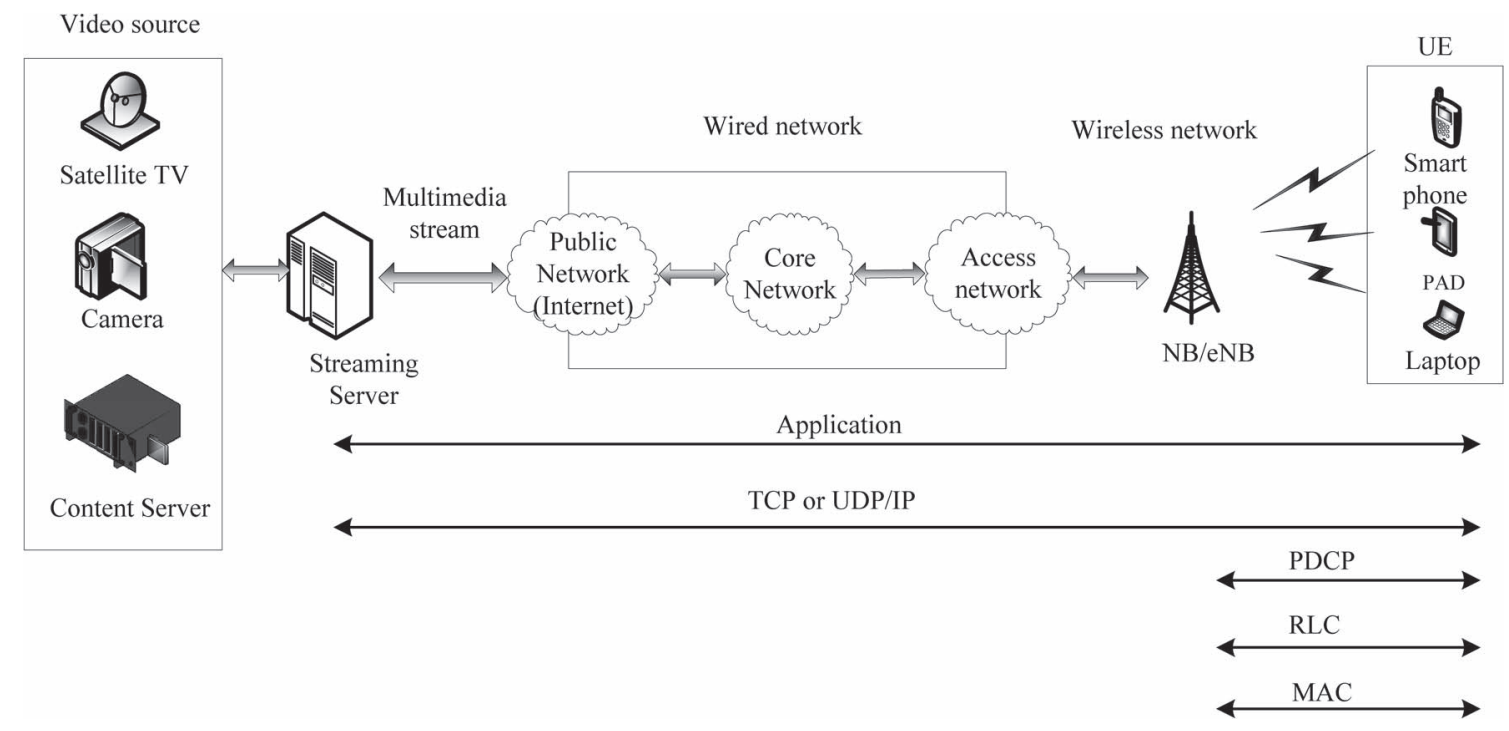

Fig. 1. Typical scenario for wireless video streaming in 3G Partnership Project (3GPP) framework.

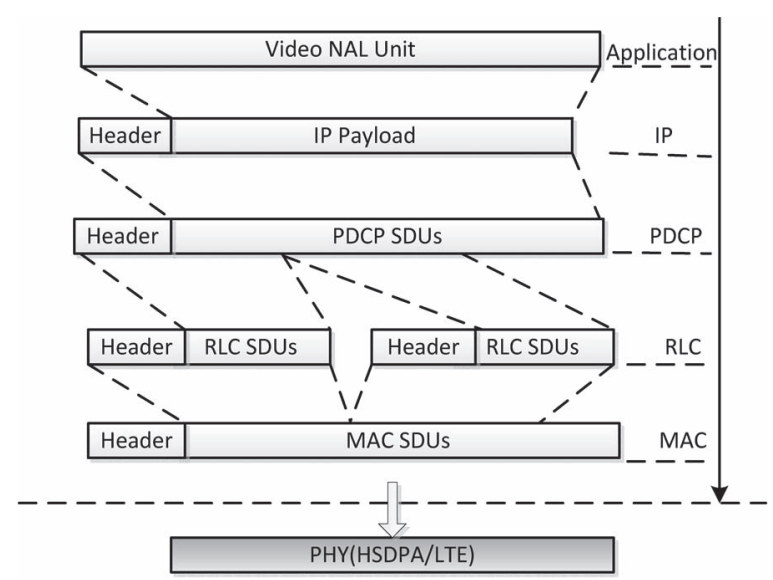

Fig. 2. Video data packetization at different layers in the 3GPP framework.

193 in Rel-9 [21]. Subsequently, we concentrate on the terminology 194 used in HSPA NB/eNB, as shown in Fig. 2. The IP/UDP/RTP 195 or IP/Transmission Control Protocol (TCP)/HTTP packet gen196 erated is encapsulated into a single Packet Data Convergence 197 Protocol (PDCP) packet that becomes an RLC service data 198 unit (SDU). Since a typical RLC SDU has a larger size than 199 an RLC protocol data unit (PDU), it has to be segmented into 200 smaller units. The length of the RLC PDU depends both on the 201 selected bearer and on the AMC mode used. The RLC PDUs are 202 forwarded to the MAC layer and are then encapsulated as MAC 203 PDUs. The main functions of the PDCP layer are robust header 204 compression and decompression, ciphering and deciphering, 205 the transfer of data, and PDCP sequence number maintenance. 206 The RLC layer in wireless systems is capable of operating 207 in both an unacknowledged mode (UM) and acknowledged 208 mode (AM), and both are capable of providing RLC PDU 209 loss detection. However, while the UM is unidirectional and 210 data delivery is not guaranteed, in the AM, automatic repeat 211 request (ARQ) is applied for reliable data transmission. The 212 main functions of the RLC layer include the transfer of upper layer PDUs; error correction relying on ARQ (only for AM); 213 and the concatenation, segmentation, and reassembly of RLC 214 SDUs, whereas the main functions of the MAC layer are 215 resource scheduling and multiplexing/demultiplexing of MAC 216 SDUs belonging to one or several logical channels into/from the 217 relevant transport blocks (TBs). By comparing the functions of 218 the RLC layer with those of the PDCP and MAC, the RLC is an 219 appropriate layer for us to construct a model for characterizing 220 the degree of matching between the network's throughput and 221 the video source bit rate. Generally, the detection of a lost 222 RLC PDU results in the loss of an entire PDCP packet; hence, 223 the encapsulated IP and the NAL unit are lost. From the 224 perspective of reacting to both the dynamics of the statistical 225 fluctuation of the teletraffic and the variable channel conditions, 226 agile bit rate adaptivity is one of the most important features 227 for seamless video streaming over wireless systems. There 228 are several ways of achieving bit rate adaptivity. For online 229 encoding applications, the bit rate adaptivity can be achieved 230 by controlling encoding parameters. For instance, H.264/AVC 231 supports these features mainly by dynamically varying the 232 quantizers but also by controlling temporal resolution. Scalable 233 Video Coding (SVC) is another technique of implementing 234 the bit rate adaptivity. It encodes the raw video clip into a 235 base layer and a number of enhancement layers with different 236 priorities. Naturally, the base layer has the highest priority since 237 it contains the video bits with the highest importance, which can 238 provide a minimum video quality. The enhancement layers with 239 lower priorities may be progressively encoded to further refine 240 the quality of the base-layer stream. This layered approach 241 of the SVC codec allows an encoded stream to be flexibly 242 prepared for meeting the bit rate constraint. In [22], the base- 243 layer rate for a given video sequence is optimized to achieve the 244 highest possible average perceived quality for heterogeneous 245 clients, whereas in [23], a distribution regime was conceived 246 for scalable videos, which guaranteed fairness for all end users. 247 In Dynamic Adaptive Streaming over HTTP (DASH), the video 248 content is partitioned into a sequence of small HTTP-based file 249 


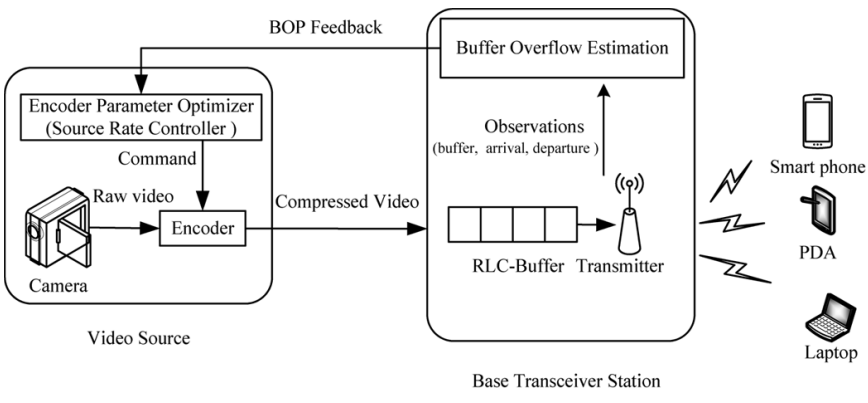

Fig. 3. Adaptive source RC framework for online video encoding.

250 segments, which are made available at a variety of different bit 251 rates. More explicitly, segments encoded at different bit rates 252 covering appropriately aligned short intervals of playback time 253 are made available. Naturally, DASH also provides a flexible 254 way of adjusting the video source bit rate. $3 \mathrm{GPP}$ has defined its 255 Rel-10 version of DASH, termed as 3GP-DASH [24], which 256 is a profile compatible with MPEG-DASH [25]. More work 257 on HTTP-based adaptive video streaming can be found in 258 [26]-[28].

259 Since the RLC buffer fullness indicates the degree of match260 ing between the source video bit rate and the channel bit rate, 261 we subsequently design a buffer fullness estimation scheme 262 where the buffer fullness estimated at the RLC layer is fed 263 back to the video source to adapt its bit rate. Without loss 264 of generality, we consider a specific scenario of the online 265 encoding $\mathrm{RC}$ relying on controlling encoding parameters, i.e., 266 dynamically changing the quantization parameters (QPs). It 267 is straightforward to extend the proposed method to both 268 SVC streaming and to HTTP-DASH scenarios, as discussed in 269 Section II-C.

\section{B. Problem Formulation}

271 The block diagram of a wireless video streaming system 272 equipped with an encoder parameter optimizer conceived to 273 control the source rate is shown in Fig. 3, which relies on a 274 camera, a video encoder, and a BTS. The camera samples the 275 video scene at certain frame scanning and forwards the frames 276 to the encoder, which forwards the compressed video to the 277 transmitter's buffer at the RLC layer for transmission. Here, we 278 assume that the channel between the video source and the BTS 279 is wired and reliable. The source RC problem is eliminating the 280 congestion in the BTS while satisfying the delay constraints. 281 Our basic philosophy is to feed back the mismatch between the 282 current source rate and the channel's affordable throughput to 283 the encoder parameter optimizer to adapt the source rate. If a 284 mismatch does occur, the encoder parameter optimizer sends 285 a command to the video encoder to adjust the video source 286 rate. To quantitatively characterize this mismatch, we define 287 BOP as the probability that the current wireless channel quality 288 provides an insufficient throughput for the current video bit rate. 289 A sufficiently low BOP indicates that we may increase the video 290 bit rate for transmission over the wireless channel to achieve a 291 higher video quality.

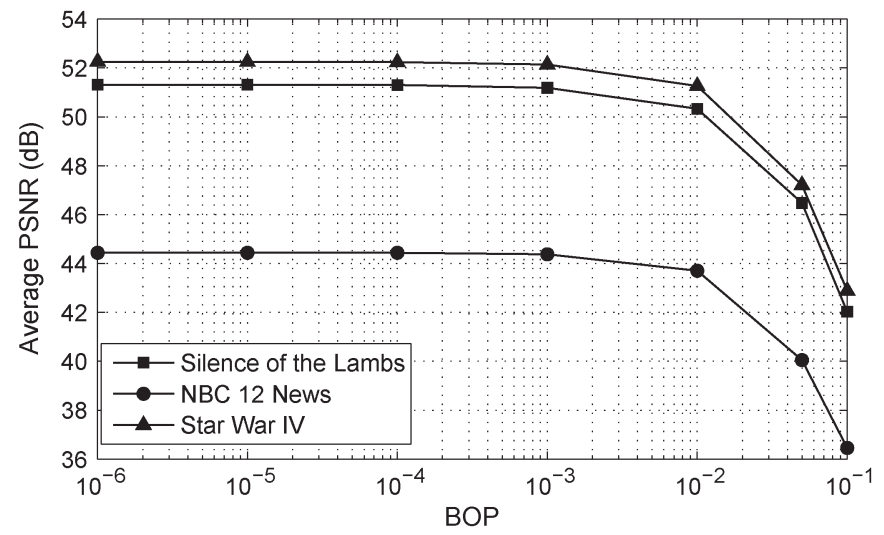

Fig. 4. Relation between $\mathrm{BOP}$ and received video quality by using three different video clips, namely, the Silence of the Lambs, NBC 12 News, and Star Wars IV.

Let $\mathcal{M} \triangleq\left\{M_{1}, \ldots, M_{L}\right\}$ be the operational mode set of the 292 video encoder. The video bit rate corresponding to the opera- 293 tional mode $M_{i}(i=1, \ldots, L)$ is denoted by $r_{i}$. Let us assume 294 that $r_{1}<r_{2}<\cdots<r_{L}$, which implies that a higher opera- 295 tional mode results in a higher video bit rate and hence achieves 296 a better video quality. The operational mode is controlled by the 297 encoder parameters. For instance, we can control the encoding 298 mode by appropriately setting the I-P-B quantization mode or 299 the target video bit rate parameter of the JM encoder. Here, 300 we consider adapting the source rate to the channel quality by 301 dynamically adjusting the operational mode (i.e., the encoding 302 parameters) of the video encoder.

303

We used transmission slots of equal duration, which are nor- 304 malized to unity. The RLC SDUs, which contain the video data 305 generated by the video encoder, arrive at the RLC buffer, and 306 they are then queued until they are transmitted. Since the data 307 are processed at packet level instead of bit granularity, we define 308 the length of the RLC buffer as the number of RLC SDUs. Let 309 $A_{n} \in \mathcal{A} \triangleq\{0, \ldots, A\}$ denote the number of RLC SDU arrivals 310 in slot $n$, where $A$ is the maximum number of RLC SDU ar- 311 rivals in a single slot, whereas $D_{n} \in \mathcal{D} \triangleq\{0, \ldots, D\}$ is defined 312 as the number of RLC SDUs transmitted in slot $n$, where $D$ is 313 the maximum number of RLS SDUs transmitted in a single slot. 314 It should be noted that an agile and sophisticated link adaptation 315 mechanism based on AMC and HARQ is applied to maintain 316 the required target bit error rate in most state-of-the-art wireless 317 communication systems such as high-speed downlink packet 318 access, 3G LTE, and WiMAX. Both AMC and HARQ rely on 319 the CQI feedback received from the mobile terminals, which 320 results in a burst-by-burst adaptive channel throughput. Hence, 321 the downlink packet departure process $D_{n}$ is assumed to be 322 an independent identically distributed (i.i.d.) sequence. Let $Q_{n} 323$ denote the buffer fullness expressed in terms of the number of 324 packets at the end of slot $n$. The dynamics of the buffer fullness 325 may be described as

$$
Q_{n}=\max \left\{Q_{(n-1)}-D_{n}, 0\right\}+A_{n} .
$$

According to Little's theorem [29], we have

$$
\bar{Q}=\lambda \bar{D}
$$


DASH Switching Command

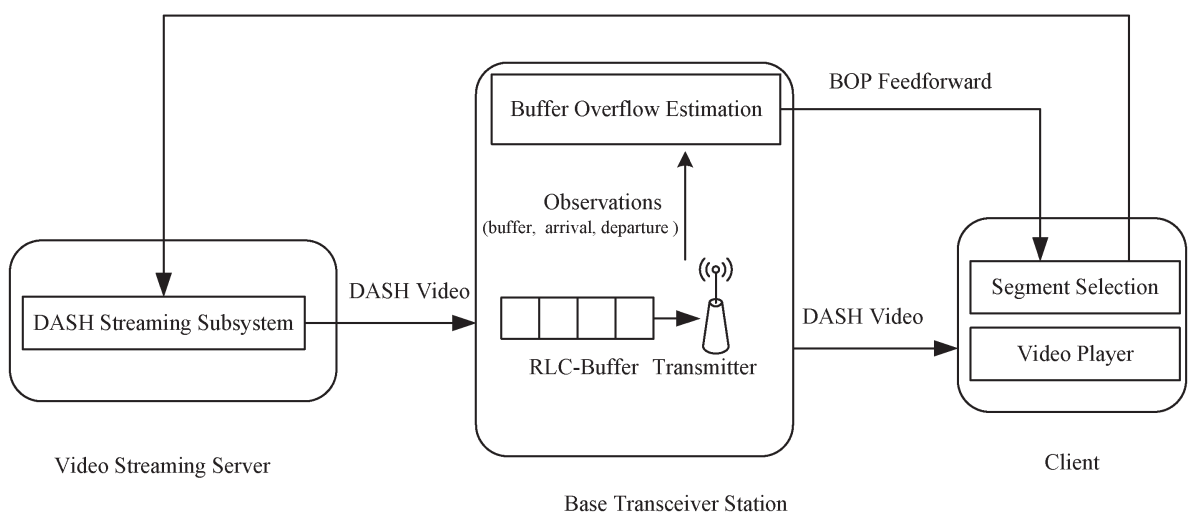

Fig. 5. Adaptive source RC framework for the DASH streaming application.

328 where $\bar{Q}$ is the average buffer fullness, $\bar{D}$ is the average delay, 329 and $\lambda$ is the packet arrival rate. This implies that, if $\lambda$ is given, 330 having a higher buffer fullness value implies a higher delay. 331 Hence, we treat the delay as being synonymous with buffer 332 fullness. Our objective is to select the best encoding mode for 333 controlling the buffer fullness to satisfy the delay constraint 334 of the specific video application. Let us formally define the $335 \mathrm{BOP}$ as

$$
p_{o}=P\left(Q>B_{h}\right)
$$

336 where $Q$ is the buffer fullness and $B_{h}$ is the buffer threshold. 337 Higher BOP implies higher buffer fullness in the near future, 338 in turn inducing a higher delay. To guarantee lip-synchronized 339 interactive low-delay video transmission, the BOP should be 340 kept low. Hence, the problem of RC may be reinterpreted as the 341 problem of selecting the best encoding mode subject to a given 342 BOP, which can be formulated as

$$
\begin{array}{ll}
\max _{m \in \mathcal{M}} & m \\
\text { s.t. } & P\left(Q^{m}>B_{h}\right)<p_{\text {qos }}
\end{array}
$$

343 where $Q^{m}$ denotes the buffer fullness corresponding to the video 344 encoding mode $m$, and $p_{\text {qos }}$ is the maximum tolerable BOP.

345 It should be noted that the queue length $P(Q>x)$ in the 346 scenario of an infinite buffer system is called an overflow 347 probability or a tail probability [30], which has been used 348 as a performance metric for an infinite buffer-aided system 349 [31]-[33]. However, practical systems have a limited buffer 350 capacity. When a video frame arrives at a full buffer, it gets 351 dropped. Hence, the packet loss probability (PLP) $P_{L}\left(B_{h}\right)$ 352 is invoked to characterize the performance of a finite-buffer353 based system having a capacity $B_{h}$ of video frames. For video 354 streaming services, the PLP is an important QoS measure, but 355 it is difficult to directly estimate the PLP of a general finite356 buffer-based system. Fortunately, a theoretical justification was 357 provided to approximate the PLP of a finite-buffer-based sys358 tem with the aid of estimating the BOP of its infinite buffer 359 counterpart [33]. Hence, we treat the BOP as being identical to 360 the PLP, which implies that the video source rate optimization 361 problem of a finite-buffer-based system having a capacity $B_{h}$ of frames can be mapped to the mathematical model of (4) and 362 (5). We have confirmed, by our simulation studies, the plausible 363 fact that a high BOP may severely degrade the QoS, which is 364 quantitatively characterized in Fig. 4 and motivates us to use 365 the BOP for the feedback control.

\section{Extensions for SVC and DASH Scenarios}

The scheme shown in Fig. 3 is a point-to-point representation 368 of our proposed framework, which may be also used for stream- 369 ing to multiple users, as shown in our forthcoming SVC/HTTP- 370 DASH streaming applications.

The framework detailed in Section II-B may be readily 372 extended to SVC and HTTP-DASH scenarios. For an SVC 373 scenario, a scalable video stream consists of a base layer 374 $l_{1}$ and $(L-1)$ enhancement layers, i.e., $\left\{l_{2}, l_{3}, \ldots, l_{L}\right\}$. The 375 operational mode $M_{i}(i=1, \ldots, L)$ is defined as the scenario 376 when the first $i$ layers are selected for transmission. Thus, the 377 source $\mathrm{RC}$ via adaptive enhancement layer selection can be also 378 formulated as the problem [see (4) and (5)]. The correspond- 379 ing video source in Fig. 3 may be also replaced by a video 380 streaming server, which maintains the sessions corresponding 381 to the multiple video users and receives the BOP feedback of 382 a specific session for adjusting the number of the transmitted 383 enhancement layers.

It should be noted that HTTP-DASH applied the pull-based 385 streaming paradigm rather than the traditional push-based 386 streaming paradigm relying on protocols such as the Real-Time 387 Streaming Protocol. The client plays the central role of driving 388 the video adaptation. To comply with the basic rule of DASH, 389 the framework for the DASH streaming applications shown 390 in Fig. 5 is based on the BOP feedforward to the client. The 391 client may then use the received BOP to select the future video 392 segments to be fetched. The DASH streaming server maintains 393 multiple sessions for the sake of supporting video streaming to 394 multiple users. In a DASH scenario, the operational mode set 395 $\mathcal{M}$ is defined as the adaptation set, where the operational mode 396 $M_{i}(i=1, \ldots, L)$ denotes streaming the chunks corresponding 397 to the $i$ th quality level. Then, the source RC via HTTP-DASH 398 can be similarly described as in (4) and (5) since a higher video 399 quality implies a higher bit rate. 
403 Naturally, the BOP estimation is a key step to successfully 404 solve the problem [see (4) and (5)]. To provide a high quality 405 of experience for a video streaming service, an occurrence of 406 buffer overflow is expected to be a rare event. The theory of 407 large deviation provides a useful way of accurately characteriz408 ing the probability of rare events. Therefore, we present a large 409 deviation-based BOP estimation model here. Although our 410 practical buffer is of finite size, the BOP of the corresponding 411 infinite-buffer-based system can be invoked to estimate the 412 PLP, as shown in [31]-[33]. Hence, we subsequently aim to 413 estimate the BOP of the corresponding infinite-buffer-based 414 system. To derive an analytical model, the BOP is estimated 415 based on the M/M/1 queueing model, which has been widely 416 applied in wireless networks [34]-[37]. The buffer at the RLC 417 is modeled as the M/M/1 queue, where $\lambda$ RLC SDUs/slot and $418 \mu \mathrm{RLC}$ SDUs/slot denote the Poissonian arrival rate and the

419 departure rate (or service rate), respectively. Let us now define 420 the large deviation theory in [19] for estimating the probability 421 of $P\left(Q_{n+N}>B_{h} \mid Q_{n}\right)$, where $Q_{n}$ is the buffer length during 422 the current time slot $n$. During the $k$ th slot, the evolution of 423 buffer length can be represented by

$$
I_{k}=A_{k}-\min \left(Q_{(k-1)}, D_{k}\right)
$$

424 Then, we have $Q_{n+N}=Q_{n}+\Sigma_{k=n+1}^{n+N} I_{k}$. Furthermore, $425 P\left(Q_{n+N}>B_{h} \mid Q_{n}\right)$ can be rewritten as

$$
\begin{aligned}
P\left(Q_{n+N}>B_{h} \mid Q_{n}\right) & =P\left(Q_{n}+\sum_{k=n+1}^{n+N} I_{k}>B_{h}\right) \\
& =P\left(\frac{1}{N} \sum_{k=n+1}^{n+N} I_{k}>a\right)
\end{aligned}
$$

426 where we have $a=\left(B_{h}-Q_{n}\right) / N$. Next, we apply the LDP to 427 the sequence $I_{n+1}, I_{n+2}, \ldots$ to derive $P\left((1 / n) \sum_{k=1}^{n} I_{k}>a\right)$. 428 The LDP can be briefly described as follows [38].

429 Definition: A sequence $X_{1}, X_{2}, \ldots$ obeys the LDP associ430 ated with rate function $I(\cdot)$ if the following conditions apply.

431 1) For any closed set $F$, we have

$$
\limsup _{N \rightarrow \infty} \frac{1}{N} \log P\left(\frac{1}{N} \sum_{i=1}^{N} X_{i} \in F\right) \leq-\inf _{a \in F} I(a) .
$$

2) For any open set $G$, we have

$$
\liminf _{N \rightarrow \infty} \frac{1}{N} \log P\left(\frac{1}{N} \sum_{i=1}^{N} X_{i} \in G\right) \geq-\inf _{a \in G} I(a)
$$

433 Obviously, $I_{k} \in \mathbb{R}(k \in[n+1, n+N])$ represents i.i.d. 434 random variables with a mean of $E\left[I_{k}\right]=\lambda-\mu$ and moment435 generating function (MGF) of $M(\theta)=E\left[e^{\theta I_{k}}\right]$ that is finite 436 in a neighborhood of 0. According to Cramér's theorem [38], $437 I_{n+1}, I_{n+2}, \ldots$ obeys the LDP associated with the rate function
$I(a)=\sup _{\theta>0}(\theta a-\log M(\theta))$, which implies that, for any 438 $a>\lambda-\mu$, we have

$$
\lim _{N \rightarrow \infty} \frac{1}{N} \log P\left(\frac{1}{N} \sum_{i=n+1}^{n+N} I_{i}>a\right)=-I(a) .
$$

It should be noted that (10) is logarithmically asymptotic. 440 Hence, when $N$ is large, the BOP can be approximated as

$$
P\left(\frac{1}{N} \sum_{i=n+1}^{n+N} I_{i}>a\right) \approx \exp [-N I(a)]
$$

Since $A_{k}$ obeys Poisson's distribution, we have

$$
E\left[e^{\theta A_{k}}\right]=\sum_{\eta=0}^{\infty} e^{\theta \eta} \frac{\lambda^{\eta}}{\eta !} e^{-\lambda}=\exp \left(\lambda e^{\theta}-\lambda\right) .
$$

Similarly, we may arrive at $E\left[e^{-\theta D_{k}}\right]=\exp \left(\mu e^{-\theta}-\mu\right)$. Then, 443 we may derive the MGF $M(\theta)$ as follows:

$$
\begin{aligned}
M(\theta) & =E\left[e^{\theta I_{k}}\right] \\
& =E\left[e^{\theta A_{k}}\right] E\left[e^{-\theta D_{k}}\right] \\
& =\exp \left(\lambda e^{\theta}+\mu e^{-\theta}-\lambda-\mu\right) .
\end{aligned}
$$

Hence, the corresponding rate function $I(a)$ can be rewritten as 445

$$
\begin{aligned}
I(a) & =\sup _{\theta>0}\left(\theta a-\lambda e^{\theta}-\mu e^{-\theta}+\lambda+\mu\right) \\
& =\left.\left(\theta a-\lambda e^{\theta}-\mu e^{-\theta}+\lambda+\mu\right)\right|_{\theta=\log \frac{a+\sqrt{a^{2}+4 \lambda \mu}}{2 \lambda}} \\
& =a \log \frac{a+\sqrt{a^{2}+4 \lambda \mu}}{2 \lambda}-\sqrt{a^{2}+4 \lambda \mu}+\lambda+\mu .
\end{aligned}
$$

If $\lambda$ and $\mu$ are given for any encoding mode $m \in \mathcal{M}$, the BOP 446 $P\left(Q_{n+N}>B_{h} \mid Q_{n}\right)$ can be estimated by using (11) and (14). 447 The problem [see (4) and (5)] can be then solved by calculating 448 the BOPs corresponding to all encoding modes in $\mathcal{M}$. However, 449 in practical situations, both $\lambda$ and $\mu$ depend on the network's 450 condition and on the source encoding mode, and they are not 451 based on prior knowledge for each encoding mode.

452

An attractive technique is applying an iterative policy to 453 solve the problem [see (4) and (5)]. Specifically, at the current 454 mode update period, we can calculate the BOP corresponding 455 to the current encoding mode by the online estimation of the 456 arrival rate $\lambda$ and the departure rate $\mu$. Although $\lambda$ and $\mu$ can 457 be estimated by counting the number of RLC SDU arrivals 458 and departures over a time period of $T$, they may change 459 over time due to time-varying network conditions and dynamic 460 encoding mode switching. The dynamic nature of $\lambda$ and $\mu$ may 461 be modeled using an autoregressive integrated moving average 462 (ARIMA) process [39] as follows:

463

$$
\begin{aligned}
& \left(1-\sum_{i=1}^{p} a_{i} D^{i}\right)(1-D)^{d_{1}} \lambda(t)=\left(1+\sum_{i=1}^{q} b_{i} D^{i}\right) \varepsilon(t) \\
& \left(1-\sum_{i=1}^{p} c_{i} D^{i}\right)(1-D)^{d_{2}} \mu(t)=\left(1+\sum_{i=1}^{q} d_{i} D^{i}\right) \mu(t)
\end{aligned}
$$


464 where $D$ is the unit time-delay operator, and $\varepsilon(t)$ is the estima465 tion error. Therefore, the task of estimating $\lambda$ and $\mu$ becomes 466 the task of estimating parameters $p, q, d_{1}, d_{2}, a_{i}, b_{i}, c_{i}$, and $d_{i}$ 467 for the ARIMA model [39]. Once $\lambda$ and $\mu$ have been estimated, 468 the corresponding BOP can be directly calculated by using (11) 469 and (14). If the BOP estimate fails to satisfy the constraint (5), 470 the source encoding rate should be decreased, thus reducing the 471 BOP. Otherwise, if the BOP is much lower than $p_{\text {qos }}$, the video 472 source bit rate may be increased, thus improving the video 473 quality. Applying this iterative encoding mode update, we can 474 iteratively solve the problem [see (4) and (5)] to find a new 475 optimal encoding mode.

476 It should be noted that the aforementioned method relies 477 on the classic M/M/1 queuing model, albeit the Poissonian 478 assumption may be not the most realistic packet arrival and 479 departure model. Moreover, our model of the BOP requires 480 estimating both $\lambda$ and $\mu$. In the next section, we propose an 481 online measurement-based RC, which no longer relies on the $482 \mathrm{M} / \mathrm{M} / 1$ queuing model and on the estimation of the RLC SDU 483 arrival rate $\lambda$ and the departure rate $\mu$.

\section{IV. ONLINE EnCODIng Mode SWITCHING-BASEd $485 \quad$ RATE CONTROL FOR VIDEO STREAMING}

486 Let the index of the current time slot be $n$ and the current 487 buffer length be $Q_{n}^{m}$, whereas $m=M_{i}$ denotes the current 488 video encoding mode. We estimate the BOP at the $(n+N)$ th $489(N>0)$ slot under the assumption of maintaining the current 490 encoding mode, where $N$ is referred to as the prediction in491 terval. Let $P_{o}^{n+N}(m)$ denote the overflow probability at slot $492(n+N)$, which is defined as

$$
P_{o}^{n+N}(m)=P\left(Q_{n+N}^{m}>B_{h}\right)
$$

493 Subsequently, we derive a BOP estimation model based on the 494 aforementioned LDP [19], and then propose an online adaptive 495 source RC for video streaming over VBR channels.

\section{A. BOP Estimation}

497 When transmitting $D_{k}$ RLC SDUs containing video data in 498 slot $k$, the increased buffer length may be expressed as

$$
I_{k}=A_{k}-\min \left(Q_{(k-1)}, D_{k}\right) .
$$

499 Since we have $0 \leq A_{k} \leq A$ and $0 \leq D_{k} \leq D$, the set of values 500 for $I_{k}$ is $\{-D, \ldots, 0, \ldots, A\}$. Let us introduce the variable $501 \pi_{i}=P\left(I_{k}=i\right)$ to denote the probability of having a buffer 502 length increase of $I_{k}=i$. For a given wired network condition, $503 A_{k}$ is determined by the video encoding mode since the en504 coded video frame size is affected by the encoding mode, and $505 D_{k}$ is related to the channel bit rate. Their difference $I_{k}$ shows 506 the instantaneous mismatch between the video bit rate and the 507 channel bit rate. Due to the time-variant RLC SDU arrivals and 508 the fluctuating channel bit rate, the sequence $I_{i}(i=1,2, \ldots)$ 509 may frequently alternate between negative and positive values.
The total increase in the buffer length during the interval 510 spanning from slot $n$ to slot $(n+N)$ is given by

$$
I^{n+N}=\Sigma_{i=1}^{N} I_{n+i}
$$

Then, the buffer length $Q_{n+N}^{m}$ at the end of the slot $(n+N) 512$ may be expressed as

$$
Q_{n+N}^{m}=Q_{n}^{m}+I^{n+N}
$$

According to (6), the BOP at slot $(n+N)$ may be rewritten as 514

$$
P_{o}^{n+N}(m)=P\left(Q_{n}^{m}+I^{n+N}>B_{h}\right)
$$

Let us now define the expected value of the average buffer 515 length increase in each of the future $N$ slots as

$$
m_{o}=E\left[\frac{\sum_{i=1}^{N} I_{n+i}}{N}\right]
$$

where $E[\cdot]$ denotes the expectation operator.Furthermore, while 517 keeping the current encoding mode fixed as $m=M_{i}$, the 518 tolerable average buffer length increase during each slot is 519 defined as

$$
a_{o}=\frac{B_{h}-Q_{n}^{m}}{N} .
$$

Having $m_{o} \geq a_{o}$ implies that there would be a high BOP after 521 $N$ time slots.

Let us now rewrite (19) as

$$
\begin{aligned}
P_{o}^{n+N}(m) & =P\left(Q_{n}^{m}+I^{n+N}>B_{h}\right) \\
& =P\left(I^{n+N} / N>\left(B_{h}-Q_{n}^{m}\right) / N\right) \\
& =P\left(\frac{\sum_{i=1}^{N} I_{n+i}}{N}>a_{o}\right) .
\end{aligned}
$$

The term $\sum_{i=1}^{N} I_{n+i} / N$ in (22) represents the average buffer 524 length change during a slot, which is jointly determined by the 525 video encoded bit rate controlled by the encoding mode and the 526 channel bit rate, whereas $a_{o}$ is the tolerable average increase in 527 the buffer length for each of the $N$ future slots, as determined by 528 the current buffer length. Therefore, the probability $P_{o}^{n+N}(m) 529$ in (22) may be viewed as an estimate of the remaining storage 530 capacity in the buffer, which may be used to smoothen the 531 fluctuation of the channel's affordable throughput in the future. 532 If the probability $P_{o}^{n+N}(m)$ exceeds a predefined threshold 533 value $p_{\text {qos }}$, we can decrease the encoding mode index to reduce 534 the video bit rate, thus decreasing both the BOP and the buffer- 535 induced delay.

536

Since $D_{k}(k=0,1, \ldots)$ are i.i.d. variables, $I_{k}(k=1,2, \ldots) 537$ are also i.i.d. random variables, which have a finite MGF of 538 $M(\theta)=E e^{\theta I_{k}}$ in the vicinity of 0 . Then, provided that $a_{o}>539$ $m_{o}$ is satisfied, according to (11), for large $N$, we have

$$
P_{o}^{n+N}(m) \approx \exp \left[-N L\left(a_{o}\right)\right]
$$


541 where

$$
\begin{gathered}
L\left(a_{o}\right)=\sup _{\theta>0}\left\{a_{o} \theta-\log M(\theta)\right\} \\
\log M(\theta)=\log \left\{\sum_{i=1-s_{D}}^{1} \pi_{i} \exp [i \theta]\right\} .
\end{gathered}
$$

542 To calculate the approximate BOP of (23), the knowledge 543 of $a_{o}, m_{o}$, and $\pi_{i}$ is required. It is straightforward to calculate $544 a_{o}$ according to (21). However, there is no prior knowledge 545 about the histogram of $I_{k}$, and hence, we cannot derive an546 alytical expressions for $m_{o}$ and $\pi_{i}$. Therefore, we have to 547 rely on their buffered history for estimating the current values 548 of these parameters using the classic sliding-window-based 549 method.

550 The observed buffer length increase/decrease sequence con551 stitutes the input of $\left\{I_{1}, I_{2}, I_{3}, \ldots\right\}$. The sliding window 552 takes into account the $N_{s}$ most recent $I_{k}$ values of $W_{n}=$ $553\left[I_{n}, I_{n-1}, \ldots, I_{n-N_{s}+1}\right]$.

554 For parameter $m_{o}$ of (20), we use the sample mean as its 555 estimate, i.e., we have

$$
\hat{m}_{o}=\frac{\sum_{i=n-N_{s}+1}^{n} I_{i}}{N_{s}} .
$$

556 Let $N_{i}(i \in\{-D, \ldots, 0, \ldots, A\})$ denote the number of 557 events when $I_{k}=i$ appears in the sliding window, which is 558 given by

$$
N_{i}=\sum_{k=n-N_{s}+1}^{n} 1\left(I_{k}=i\right)
$$

559 where $1(\cdot)$ is an indicator function. Then, the relative frequency 560 of encountering $I_{k}=i$ may be estimated as

$$
\hat{\pi}_{i}(n)=\frac{N_{i}}{N_{s}}
$$

561 which can be applied in (25) to estimate the BOP.

\section{B. Online Source RC Algorithm}

563 The RC strategy advocated will be discussed in the context of 564 three scenarios according to both the current buffer length $Q_{n}$ 565 and the average buffer length increase per slot $\hat{m}_{O}$ as follows.

566 1) $Q_{n}^{m} \geq B_{h}$ : This implies that the current buffer length is 567 above the threshold, which will impose an undesirable delay of 568 the video frame. Therefore, we should reduce the video bit rate 569 by decreasing the encoding mode index as

$$
m=M_{\max \{i-1,1\}} .
$$

570 2) $Q_{n}^{m}<B_{h}$ and $\hat{m}_{o} \geq a_{o}$ : In this scenario, although the 571 buffer length is under the threshold $B_{h}$, its average increase per 572 slot $\hat{m}_{o}$ exceeds the tolerable average increase $a_{o}$ of the buffer 573 length per slot during the forthcoming $N$ slots. This implies 574 that, at the current buffer length increase rate, the buffer length 575 will become higher than $B_{h}$ after $N$ slots. Therefore, in this case, the current video bit rate should be decreased to reduce 576 the BOP. Then, we can adjust the encoding mode according 577 to (29).

3) $Q_{n}^{m}<B_{h}$ and $\hat{m}_{o}<a_{o}$ : In this case, the current buffer 579 length is under the threshold $B_{h}$, and the average buffer length 580 increase per slot $\hat{m}_{o}$ is within the range defined by the capacity 581 $a_{o}$. Nevertheless, this does not imply that no buffer overflow 582 will occur in the forthcoming $N$ slots because $\hat{m}_{O}$ is the 583 average buffer length increase per slot, which cannot directly 584 characterize the buffer length increase in a certain time slot. 585 Hence, there is still a chance of buffer overflow. Fortunately, 586 since we have $a_{o}>\hat{m}_{o}$, buffer overflows remain a rare event. 587 According to the large deviation-based probability estimation 588 model of (23), for a sufficiently large $N$, the BOP may be 589 approximated as

$$
\hat{P}_{o}^{n+N}(m)=\exp \left[-N L\left(a_{o}\right)\right]
$$

An online measurement-based estimation method was pre- 591 sented in the previous section to calculate the BOP. Owing 592 to the exponential decay of the estimated BOP probability 593 with $N$, we can set $N$ to a moderate value for the sake of 594 acquiring an accurate BOP estimation instead of requiring a 595 large $N$.

Our proposed RC algorithm aims to adjust the encoding 597 mode to satisfy the BOP QoS requirement, i.e., $p_{\mathrm{qos}}$. If we have 598 $\hat{P}_{o}^{n+N}(m) \geq p_{\mathrm{qos}}$, this implies that the current encoding mode 599 index is too high to keep the BOP below $p_{\text {qos }}$. Therefore, we 600 should decrease the encoding mode index to reduce the video 601 bit rate, thus reducing the BOP. The future encoding mode 602 index is adjusted as $m=M_{\max \{i-1,1\}}$.

By contrast, if we have $\hat{P}_{o}^{n+N}(m)<p_{\text {qos }}$, the currently 604 affordable bit rate of the channel may be able to support a 605 higher video bit rate. Hence, we should increase the encoding 606 mode index to provide an improved video quality for the sake 607 of fully exploiting the attainable bit rate of the channel. To 608 achieve this, we define a threshold $p_{T}\left(<p_{\text {qos }}\right)$ for the BOP. 609 If $\hat{P}_{o}^{n+N}(m)<p_{T}$ is encountered consecutively $K>0$ times, 610 we will increase the encoding mode index according to

$$
m=M_{\min }\{i+1, L\} .
$$

The reason for requiring $K$ consecutive threshold viola- 612 tion occurrences to trigger an encoding mode index adjust- 613 ment is that this prevents frequent adjustments of the encod- 614 ing mode, which would result in perceivable video quality 615 fluctuations.

The RC regime is summarized in Algorithm 1. Although the 617 estimated average channel throughput was directly fed back to 618 the BTS to control the source rate in [18], this technique does 619 not characterize the burst-by-burst adaptive channel throughput 620 on a sufficiently fine timescale, which, hence, fails to guarantee 621 a low delay for the video packets. By contrast, Algorithm 1622 relies on the LDP of [19] to estimate the BOP, when the buffer 623 overflow is a rare event, and applies the BOP constraint to 624 trigger the source RC, thus achieving a low delay for video 625 packets. 
Algorithm 1 Online measurement-based adaptive rate control algorithm for streaming video over VBR channels.

\section{C. Discussion}

647 Previously, the proposed solution was discussed in the con648 text of a single user requesting a single video stream. However, 649 it may be also extended to the scenario where multiple users 650 having a different link quality desire the same video. A simple 651 solution is for the video server to create a dedicated encoder 652 instance for each encoding mode, where multiple video streams 653 are generated by the encoders with the aid of different encoding 654 modes. Then, based on the feedback triggered by the proposed 655 method from the BTS, the video server may select an appropri656 ate video stream for each user associated with a different link 657 quality.

\section{Performance Evaluation}

659 Here, we characterize the performance of our online 660 measurement-based adaptive RC (MBARC) algorithm. We first 661 describe our simulation setup, including the network model 662 and the video sequences employed. Then, the metrics used for 663 performance evaluation are described. Finally, our simulation 664 results are presented and analyzed. In the simulations, we 665 also implemented the heuristic online adaptive RC (HOARC) 666 algorithm in [18] and an offline method to provide pertinent 667 performance comparisons to cutting-edge benchmarkers. The 668 offline method simply relied on all the encoding modes and 669 selected the mode having the best performance as the perfor670 mance benchmark.

\section{A. Simulation Setup}

672 We consider an HSPA network [1] relying both on AMC and 673 HARQ. We assume a UE (UE in HSPA parlance) belonging 674 to category 10. According to the HSPA specifications [2], the $675 \mathrm{CQI}$ value ranges from 0 to 30 , and the corresponding TB sizes

TABLE I

Properties of the Video Sequences

\begin{tabular}{|c|c|}
\hline Encoder & H.264 Full \\
\hline Resolution & $\begin{array}{l}\text { CIF }(352 \times 288): \\
\text { Silence of the Lambs } \\
\text { NBC } 12 \text { News } \\
\text { D-1(704 } \times 576) \text { : } \\
\text { The Shawshank Redemption }\end{array}$ \\
\hline Bit Rate & Variable Bit Rate (VBR) \\
\hline Number of Frames & 45,000 \\
\hline GoP Size & 16 \\
\hline Frames Rate & 30fps \\
\hline Video Duration & 25 minutes \\
\hline No. B frames between I/P frames & 1 \\
\hline
\end{tabular}

are $0,137, \ldots, 25558$ bits, respectively. In HSPA, there are 15676 time-division multiplex slots per 10-ms frame. Three 10/15 $=677$ $2 / 3$ ms slots form a so-called transmission time interval (TTI) 678 of 2-ms duration, where only one user is allowed to transmit 679 with the aid of multiple spreading codes per TTI. Therefore, 680 the time-varying number of users may result in a time-varying 681 number of TTIs being assigned to each user, which, in turn, 682 leads to a time-varying throughput for each user. Therefore, we 683 simulated a multiuser scenario, where the maximum number 684 of concurrently communicating users was set to $U=8$, and 685 the new user arrival process follows a Markov process with an 686 arrival rate of $\lambda=10^{-4}$ per TTI. The service rate was assumed 687 to be $v=1.5 \times 10^{-5}$ per TTI. A round robin scheme was 688 applied to schedule the transmissions of the users. Then, our 689 proposed strategy is applied for one of the users to implement 690 its online source RC. Consider an i.i.d. Rayleigh channel for 691 the target user, where the received SNR $s$ is an exponentially 692 distributed random variable described by the probability density 693 function of $f(s)=(1 / \gamma) e^{-(s / \gamma)}$ having an average of $\gamma$. We 694 applied the SNR (in decibels)-to-CQI mapping in [40], i.e., 695

$$
\mathrm{CQI}=\lfloor\mathrm{SNR}+4.5\rfloor .
$$

According to the specifications, the CQI reporting cycle is 696 defined as 1, 2, 4, 5, 10, 20, 40, and 80 TTIs. In the simulations, 697 we set the CQI reporting cycle to four TTIs.

\section{B. Video Sequences Used for Performance Evaluations}

Three different video sequences are used in our simulations, 700 namely, the "Silence of the Lambs" clip, the "NBC 12 News" 701 clip [17], and the "Shawshank Redemption" clip, scanned at 30702 frames/s and encoded by the H.264 codec. The two former clips 703 have a Common Intermediate Format (CIF) resolution, whereas 704 the last clip has a D-1 resolution. The duration of the video 705 sequence is $25 \mathrm{~min}$, corresponding to 45000 video frames, 706 where a GOP is constituted by 16 frames. The properties of 707 these video sequences are listed in Table I. Here, the encoder 708 mode is denoted by $\left(m_{1}, m_{2}, m_{3}\right)$, where $m_{1}, m_{2}$, and $m_{3} 709$ represent the quantization scales for the I, P, and B frames, re- 710 spectively. The operational modes are listed as follows: $M 1=711$ $(10,10,12), \quad M 2=(16,16,18), \quad M 3=(22,22,24), \quad M 4=712$ $(24,24,26), \quad M 5=(28,28,30), \quad M 6=(34,34,36), \quad M 7=713$ 


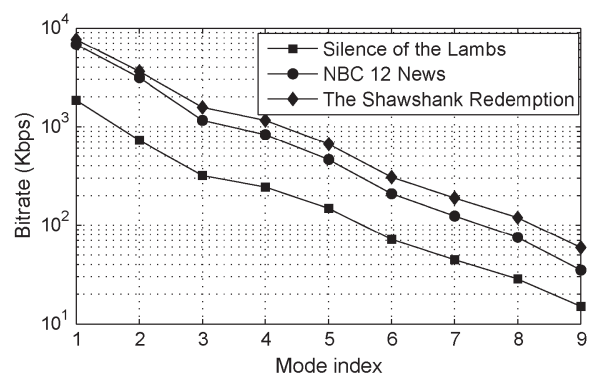

(a)

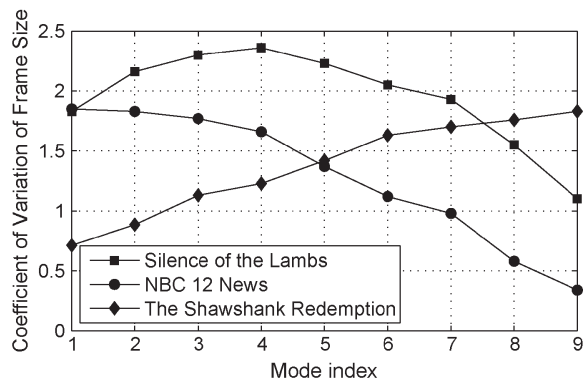

(b)

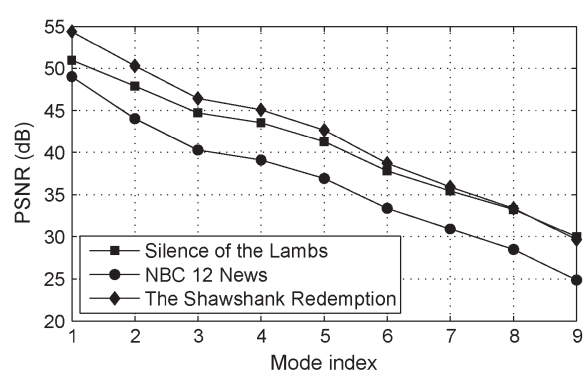

(c)

Fig. 6. Statistics of the video sequences. (a) Mean bit rate. (b) Coefficient of variation of frame size. (c) Mean PSNR.

$714(38,38,40), M 8=(42,42,44)$, and $M 9=(48,48,50)$. Fig. 6 715 characterizes the bit rate, the frame-size variation coefficient, 716 and the peak SNR (PSNR) statistics of the video sequences 717 in the different encoding modes, with the frame-size variation 718 coefficient being defined in [41] as follows:

$$
C o V_{X}^{q}=\frac{1}{\bar{X}_{N}^{q}} \sqrt{\frac{1}{N-1} \sum_{n=0}^{N-1}\left(X_{n}^{q}-\bar{X}_{N}^{q}\right)^{2}}
$$

719 where $X_{n}^{q}$ denotes the frame size of video frame $n$ encoded 720 with the QP $q$, and $\bar{X}_{N}^{q}$ is the average frame size of the $N$ video 721 frames. In the first two simulations, the former two CIF clips 722 were used for basic performance investigations, whereas in the 723 rest of the simulations, the D-1 clip was used to characterize the 724 achievable performance in the case of higher resolution videos.

\section{C. Performance Metrics}

726 In the simulations, each RLC SDU carried the bits of video 727 frames. To satisfy the tolerable delay constraint of a specific 728 video application, the RLC SDUs are dropped from the buffer 729 when their delay exceeded the threshold of $l$ milliseconds. To 730 evaluate the quality of a received video sequence, an objective 731 video quality evaluation model similar to [43] is adopted, which 732 is defined by the rate of dropped video frames (DVFRs) that are 733 undecodable at the MS, i.e., by

$$
\mathrm{DVFR}=1-\frac{N_{\mathrm{dec}}}{N_{\text {total }-I}+N_{\text {total }-P}+N_{\text {total }-B}}
$$

734 where $N_{\text {dec }}$ is the total number of decodable frames, including 735 all three types of frames. The decoding dependence values 736 between different types of frames are also considered in count737 ing the decodable frames. A lower DVFR value implies that a 738 better quality is perceived by the recipient. Since the proposed 739 adaptation method uses different bit rates, when configured 740 for adapting the throughput and the quality, we also apply the 741 average PSNR as a further performance metric.

742 All the results were averaged over 100 independent sim743 ulation runs. To characterize the performance improvement 744 of our MBARC, we also conducted independent simulations 745 for each encoder mode, and the best results were selected as 746 performance benchmarks.

\section{Simulation Results}

1) Performance for Different Delay Thresholds: In the first 748 experiment, the parameters of the proposed online MBARC 750 algorithm were set as follows: length of the sliding window 751 $N_{s}=80$, prediction interval $N=80$, buffer length threshold 752 $B_{h}=16, p_{\text {qos }}=10^{-5}, p_{T}=10^{-10}$, and $K=32$. The initial 753 mode index of the encoder is $M_{9}$, which has the lowest video 754 bit rate. The MBARC was activated every eight video frame 755 intervals. Its performance was investigated for the delay thresh- 756 olds of $\{60,100,140,180$, and $220 \mathrm{~ms}\}$, which cover the 757 delay requirements of various video applications. For example, 758 the delay limit of $60 \mathrm{~ms}$ is applicable to lip-synchronized 759 real-time interactive video conferencing applications, whereas 760 the delay limit of 100 or $140 \mathrm{~ms}$ is applicable to wireless 761 video surveillance, and finally, 180 and $220 \mathrm{~ms}$ are for digital 762 television broadcast or video-on-demand services. Fig. 7(a) and 763 (b) shows the DVFR and the average PSNR of Silence of the 764 Lambs and NBC 12 News for different delay thresholds at the 765 average channel SNR of $\gamma=20 \mathrm{~dB}$. It can be observed in Fig. 7766 that, as the encoder mode index increases, the DVFR decreases 767 owing to the reduced source rate. For Silence of the Lambs, 768 the DVFR of the operational mode $M 6$ is similar to the DVFR 769 of MBARC, but our MBARC improves the average PSNR by 770 about $3 \mathrm{~dB}$. For NBC 12 News, our MBARC and $M 7$ have 771 a similar DVFR, but MBARC improves the average PSNR by 772 about $1 \mathrm{~dB}$. This implies that the proposed MBARC is capable 773 of adaptively adjusting the encoder mode when the source rate 774 is temporarily higher than the affordable channel rate. 775

2) Performance for Different Channel SNRs: To investi- 776 gate the proposed MBARC's source rate adaptation capabil- 777 ity for different channel qualities, we conducted experiments 778 at different average channel SNRs, namely, at $\gamma=\{12,16,779$ $20,24$, and $28 \mathrm{~dB}\}$. The buffer length threshold was set to 780 28, whereas the remaining MBARC parameters were the same 781 as in the first experiment. We set the maximum delay, which 782 triggers dropping of the frames in the buffer to $200 \mathrm{~ms}$. For 783 each average channel SNR considered, we use an offline pro- 784 cedure to select the best encoding mode, which maximizes 785 the average PSNR, while maintaining a DVFR similar to that 786 of MBARC. The simulation results shown for the MBARC, 787 HOARC, and offline mode selection method were plotted in 788 Fig. 8(a) and (b). The results recorded in Fig. 8(b) for dif- 789 ferent average channel SNRs using the offline method were 790 marked with (Mode). Fig. 8(b) demonstrates the average PSNR 791 

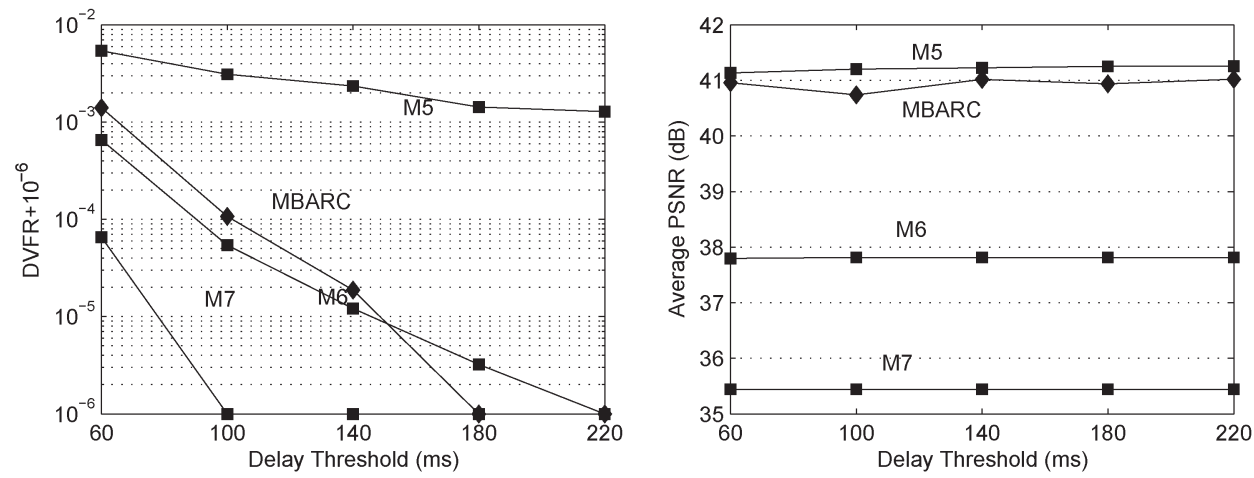

(a)
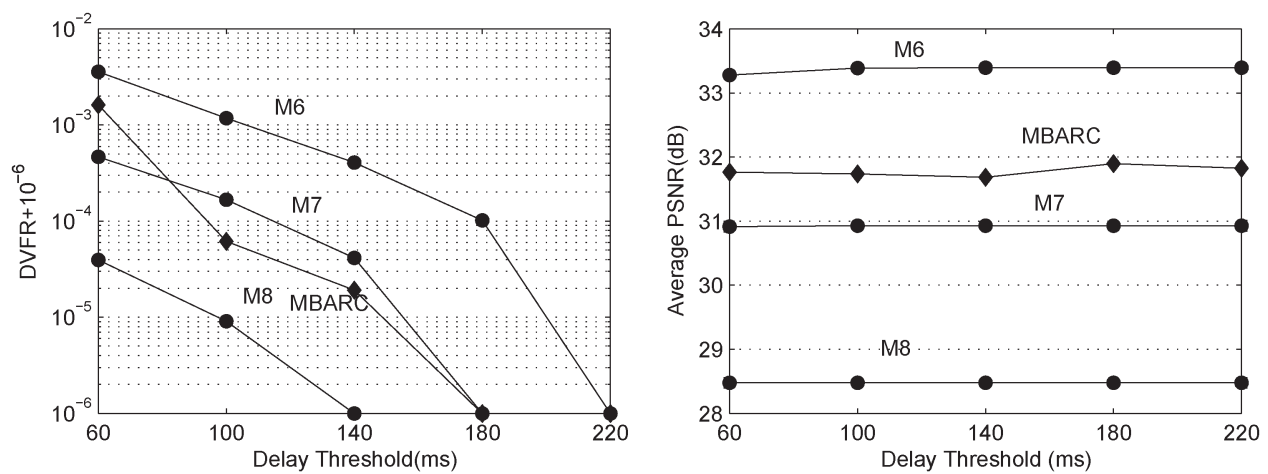

(b)

Fig. 7. (a) DVFR and Average PSNR of Silence of the Lambs for different delay thresholds with average channel SNR of $20 \mathrm{~dB}$. (b) DVFR and Average PSNR of NBC 12 News for different delay thresholds with average channel SNR of 20 dB. (a) Silence of the Lambs. (b) NBC 12 News.

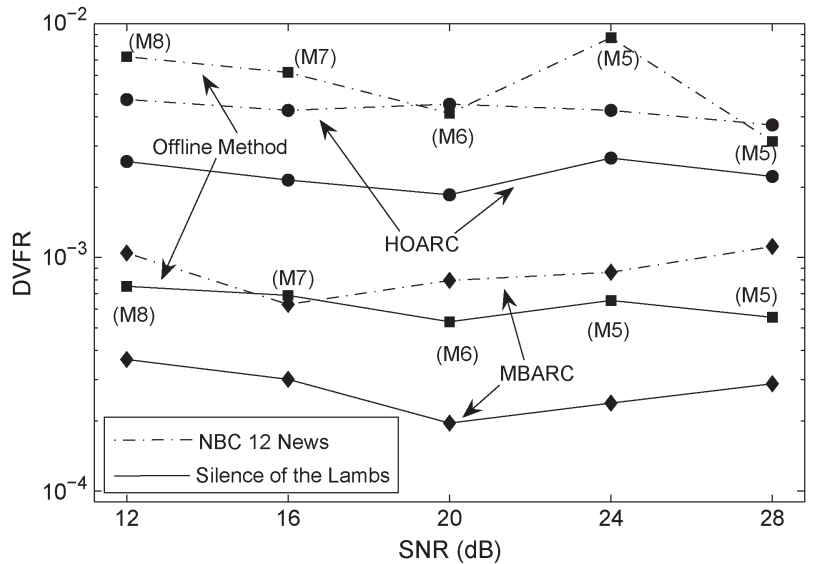

(a)

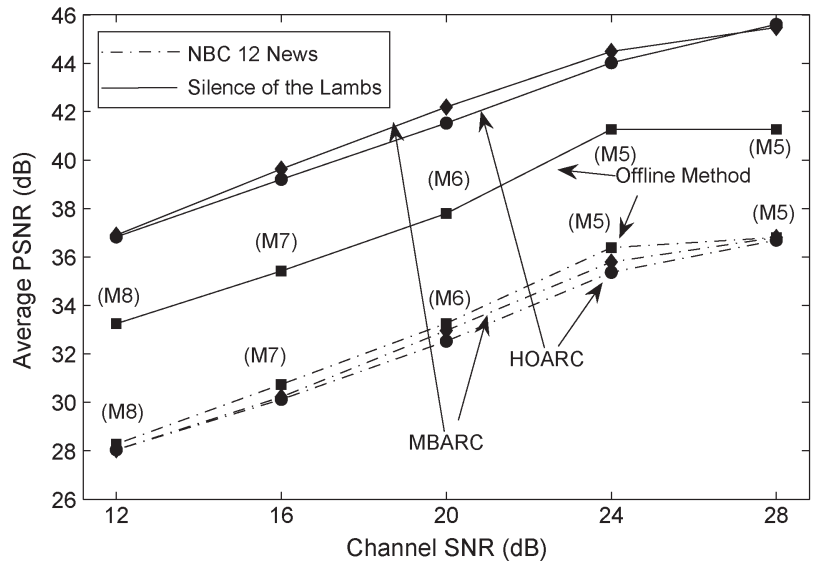

(b)

Fig. 8. (a) DVFR for different average channel SNRs of Rayleigh channel. (b) PSNR for different average channel SNRs of Rayleigh channel. The note in () represents the corresponding encoder mode for the offline method. (a) DVFR. (b) Average PSNR.

792 improvement of MBARC over the offline method, whereas its 793 DVFR remains lower than that of the offline method. This 794 demonstrates that MBARC is capable of adaptively accom795 modating different channel qualities by adjusting the encoding 796 mode without any prior knowledge of the channel quality. Al797 though Fig. 8(b) also shows that the HOARC technique in [18] 798 is capable of adapting to time-variant channel conditions and 799 approaching the PSNR performance of the proposed MBARC, 800 it suffers from a much higher DVFR than that of the MBARC 801 technique, as indicated in Fig. 8(a). The basic reason for this is elaborated as follows. The linear $\mathrm{AR}(1)$ of the HOARC 802 technique may not be appropriate for estimating the average 803 channel throughput since HSPA applies near-instantaneously 804 adaptive burst-by-burst transmissions. Furthermore, the aver- 805 age channel throughput cannot fully characterize the specific 806 near-instantaneously adaptive channel throughput at a certain 807 transmission time instant, which, in turn, increases the delay 808 of a specific packet and results in an increased probability of 809 delay constraint violation. By contrast, the proposed MBARC 810 technique is based on a BOP constraint, which may guarantee a 811 


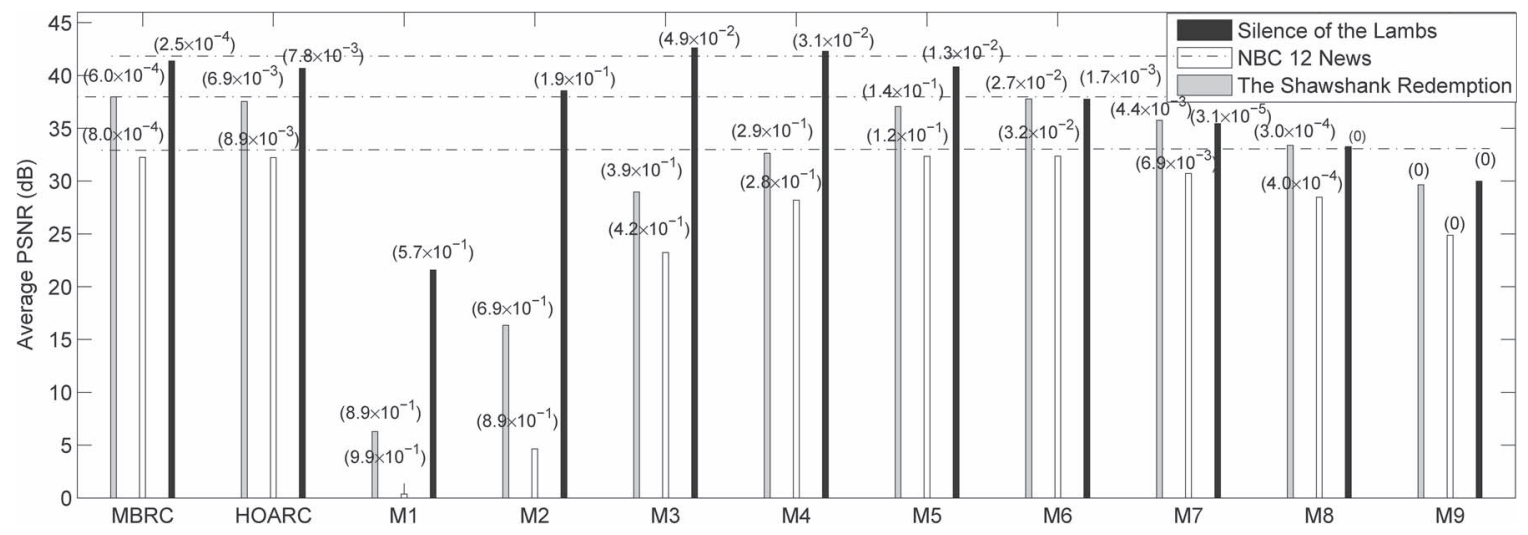

Fig. 9. Average PSNR for dynamic average channel SNRs for Rayleigh channel. The number in () represents the corresponding DVFR.

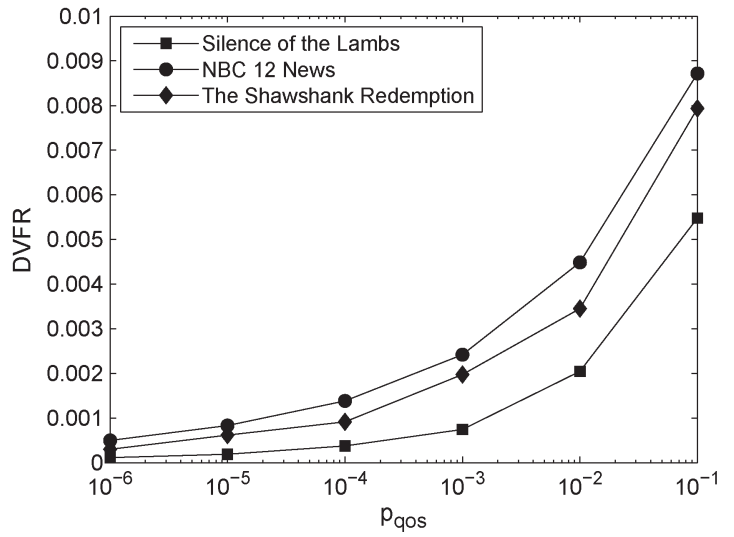

(a)

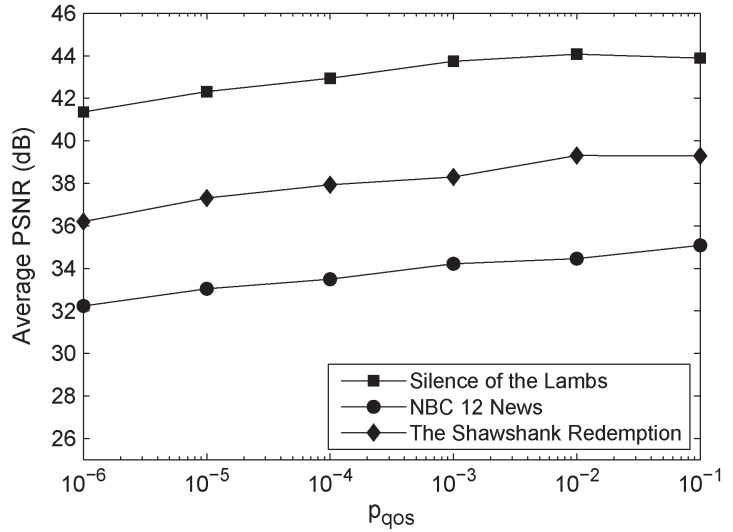

(b)

Fig. 10. (a) DVFR for different overflow probability thresholds $p_{\mathrm{qos}}$. (b) PSNR for different overflow probability thresholds $p_{\mathrm{qos}}$. (a) Silence of the Lambs. (b) NBC 12 News.

812 low delay for the video packets. The application of the LDP [19] 813 assists MBARC in achieving an accurate estimation of BOP 814 and, thus, improves the DVFR performance.

815 3) Performance for Dynamic Channel Quality: The previous 816 investigations were conducted in a static environment by fixing 817 the average channel SNR. Next, we investigate the performance 818 of the MBARC in time-varying scenarios. The initial average 819 channel SNR was set to $24 \mathrm{~dB}$. At the instant of the 18000 th 820 video frame interval, the average channel SNR was suddenly 821 changed to $20 \mathrm{~dB}$. Then, at the instant of the 36000th frame 822 interval, it was further reduced to $16 \mathrm{~dB}$. All the MBARC 823 parameters remained the same as in the previous experiment. 824 Fig. 9 shows the corresponding simulation results, where each 825 bar is marked with "(DVFR)" to characterize the corresponding 826 DVFR. It is shown that the DVFR of our MBARC is lower 827 than that of $M 6$ for Silence of the Lambs and that it improves 828 the PSNR by more than $2 \mathrm{~dB}$ in comparison with that of $829 M 6$. For NBC 12 News, although $M 8$ has a similar DVFR 830 to that of our MBARC, its average PSNR is $2 \mathrm{~dB}$ lower than 831 that of the proposed MBARC. This benefit is the result of 832 the MBARC's adaptability, which adjusts the encoder mode 833 online for VBR encoding to accommodate diverse dynamically 834 fluctuating propagation environments. By contrast, for a fixed 835 encoder mode, having a low channel SNR may inflict frame 836 dropping events, resulting in low video quality, whereas at high
SNRs, it fails to promptly decrease the video rate to improve 837 the video quality in a timely manner. Similarly, MBARC is 838 capable of adaptation and achieves an improved performance 839 for the Shawshank Redemption clip having a higher resolution. 840 Observe in Fig. 9 that the HOARC technique [18] approaches 841 the PSNR performance of the proposed MBARC, but it exhibits 842 a significantly higher DVFR than MBARC. This result also 843 illustrates the benefit of the explicit BOP constraint in the 844 problem formulation (4) and (5) and that of applying the LDP 845 for accurately estimating BOP.

4) Performance Sensitivity of the Proposed Algorithm: The 847 performance of the proposed method relies on parameters $N, 848$ $N_{s}$, and $K$, as shown in Algorithm 1. Hence, this section 849 investigates their effect on the performance of the proposed 850 method. In the related experiments, one of these three param- 851 eters was set to different values, whereas the other parameters 852 of our MBARC were the same as those in 2). The average SNR 853 value of our Rayleigh channel model was fixed to $20 \mathrm{~dB}$. The 854 remaining parameters of the channel model were set as in 2). 855

Fig. 10 shows our simulation results of different BOP thresh- 856 olds $p_{\text {qos }}$ for the three clips. We may observe in Fig. 10 that 857 a reduced overflow probability threshold provides a reduced 858 DVFR. This is because a reduced probability threshold is 859 capable of activating timely adjustments of the encoder mode 860 to reduce the BOP. On the other hand, we can also observe in 861 


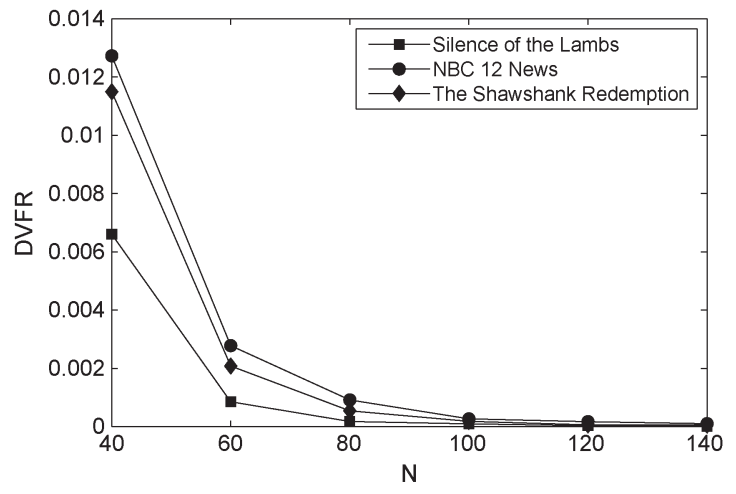

(a)

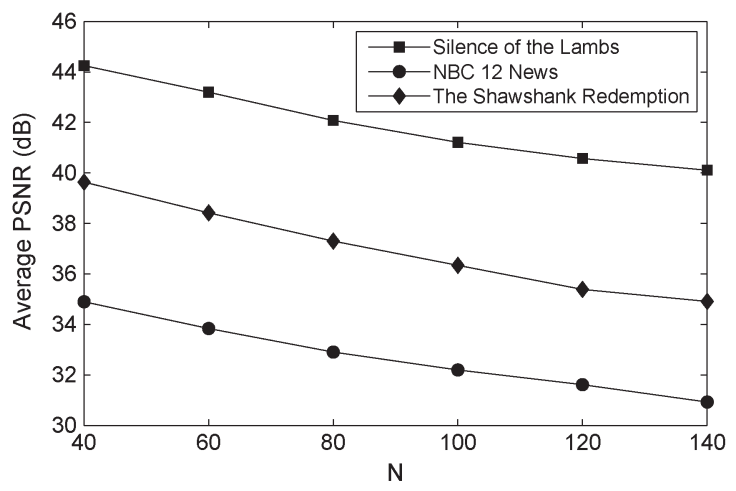

(b)

Fig. 11. (a) DVFR for different prediction intervals $N$. (b) PSNR for different predicting intervals $N$. (a) DVFR. (b) PSNR.

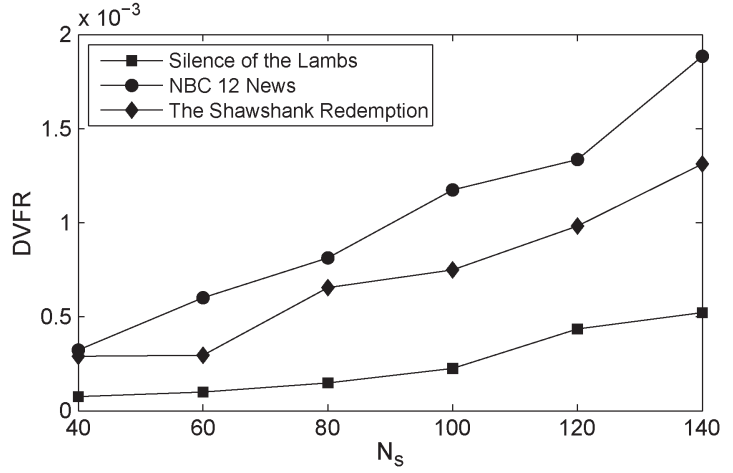

(a)

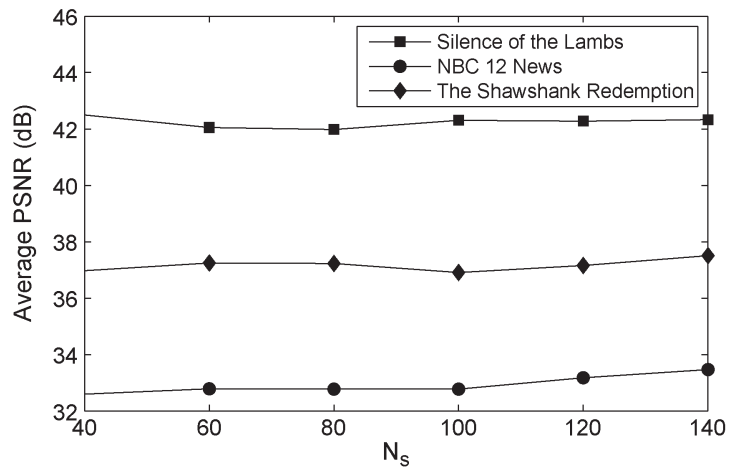

(b)

Fig. 12. (a) DVFR for different lengths of the sliding window $N_{s}$. (b) PSNR for different lengths of the sliding window $N_{s}$. (a) DVFR. (b) PSNR.

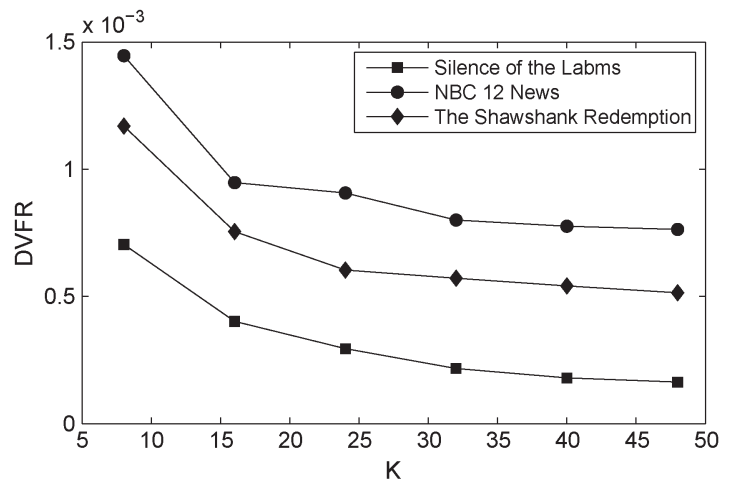

(a)

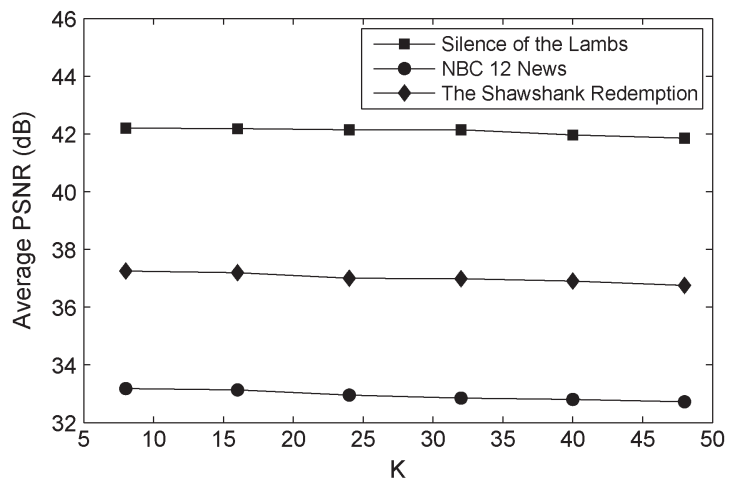

(b)

Fig. 13. (a) DVFR for different values of $K$. (b) PSNR for different values of $K$. (a) DVFR. (b) PSNR.

862 Fig. 10 that, as the BOP threshold decreases, the average PSNR 863 increases. This is caused by the lower encoding mode index 864 used to increase the video quality when the BOP threshold is 865 higher. It should be noted that, although the DVFR is related 866 to the BOP, their definitions are different. Hence, the value 867 of DVFR is not the same as $p_{\text {qos }}$, as shown in Fig. 10(a). 868 Fortunately, we may achieve an acceptable DVFR by setting $869 p_{\mathrm{qos}}$ to an appropriate value such as $10^{-5}$.

870 Fig. 11 plots the simulation results for different prediction 871 intervals $N$ for the three clips. Fig. 11(a) shows that the DVFR 872 is rapidly reduced upon increasing $N$, whereas Fig. 11(b) shows 873 that the average PSNR is decreased as $N$ is increased. The basic reason is elaborated as follow. According to (30), a large 874 value of $N$ leads to a more accurate BOP estimate, which 875 assists the proposed method to trigger appropriate adjustments 876 of the encoding mode and thus to reduce the DVFR. The result 877 shows that $N \geq 80$ is appropriate for achieving an acceptable 878 performance for the proposed method.

Fig. 12 shows the performance sensitivity of the proposed 880 method to the sliding-window duration. It is shown in Fig. 12(a) 881 that a larger value of $N_{s}$ may result in a higher DVFR. This 882 may be caused by the reduced sensitivity of the buffer variance 883 when using a larger $N_{s}$ for estimating $\hat{m}_{o}$ according to (26). 884 Fig. 12(b) shows that $N_{s}$ has only a modest effect on the PSNR. 885 
886 Fig. 13 shows the sensitivity of the performance to parameter $887 K$. The proposed method triggers a video bit rate increase via 888 adjusting the encoding mode only when the threshold violation 889 of $\hat{P}_{o}^{n+N}<p_{T}$ is encountered consecutively $K$ times. There890 fore, having a larger $K$ implies a more conservative adjustment 891 policy, which leads to a lower DVFR and to a marginally 892 reduced PSNR, which are shown in Fig. 13(a) and (b).

\section{CONCLUSION}

894 An online adaptive source RC regime has been proposed for 895 streaming videos in HSPA and LTE-like VBR downlink sce896 narios. Large deviation theory was invoked to derive the online 897 measurement-based BOP model, applied at the RLC layer in the $898 \mathrm{BS}$. The advantage of the resultant algorithm is that it exploits 899 the online observations of buffer length and its variation for $900 \mathrm{RC}$, requiring no prior knowledge of the channel variations 901 and video characteristics. Our simulation results recorded in 902 multiuser scenarios demonstrated that the proposed algorithm 903 is capable of accommodating the channel quality variations, 904 which may be beneficial in HSPA and LTE-style environments.

905

906

907

908

909

910

911

912

913

914

915

916

917

918

919

920

921

922

923

924

925

926

927

928

929

930

931

932

933

934

935

936

937

938

939

940

941

942

943

944

945

946

947

948

949

\section{REFERENCES}

1] L. Hanzo, J. Blogh, and S. Ni, 3G, HSPA and FDD Versus TDD Networking: Smart Antennas and Adaptive Modulation. Piscataway, NJ, USA: Wiley/IEEE Press, 2008.

2] Third-Generation Partnership Project (3GPP), "Physical layer procedures (FDD)," ETSI, Sophia Antipolis, France, 3GPP TS25.214 V6.2.0, 2004.

3] M. Chen and A. Zakhor, "Rate control for streaming video over wireless," IEEE Wireless Commun., vol. 12, no. 4, pp. 32-41, Aug. 2005.

[4] T. Chang and Y.-Q. Zhang, "A new rate control scheme using quadratic rate-distortion modeling," IEEE Trans. Circuits Syst. Video Technol., vol. 7, no. 1, pp. 246-250, Feb. 1997.

5] Z. G. Li et al., " A unified architecture for real-time video-coding systems," IEEE Trans. Circuits Syst. Video Technol., vol. 13, no. 6, pp. 472-487, Jun. 2003.

[6] Y. Liu, Z. G. Li, and Y. C. Soh, "Rate control of H.264/AVC scalable extension," IEEE Trans. Circuits Syst. Video Technol., vol. 18, no. 1, pp. 116-121, Jan. 2008.

7] "Joint Video Team Software JM 18.0." [Online]. Available: http://iphome.hhi.de/suehring/tml/

8] Z. Li et al., "Adaptive basic unit layer rate control for JVT," presented at the 7th Meeting, Pattaya II, Pattaya, Thailand, Mar. 2003, Paper JVTG012r1.

[9] L. Hanzo, P. Cherriman, and J. Streit, Video Compression and Communications. Hoboken, NJ, USA: Wiley, 2007.

0] Y. Huang, S. Mao, and S. F. Midkiff, "A control-theoretic approach to rate control for streaming videos," IEEE Trans. Multimedia, vol. 11, no. 6, pp. 1072-1081, Oct. 2009.

11] H. L. Lin, T. Y. Wu, and C. Y. Huang, "Cross layer adaptation with QoS guarantees for wireless scalable video streaming," IEEE Commun. Lett., vol. 16, no. 9, pp. 1349-1352, Sep. 2012.

12] N. Changuel, N. Mastronarde, M. Van der Shaar, B. Sayadi, and M. Kieffer, "Adaptive scalable layer filtering process for video scheduling over wireless networks based on MAC buffer management," in Proc. IEEE ICASSP, May 2011, pp. 2352-2355.

13] A. Dua, C. W. Chan, N. Bambos, and J. Apostolopoulos, "Channel, deadline, and distortion $\left(C D^{2}\right)$ aware scheduling for video streams over wireless," IEEE Trans. Wireless Commun., vol. 9, no. 3, pp. 1001-1011, Mar. 2010.

14] H. Zhang, Y. Zheng, M. A. Khojastepour, and S. Rangarajan, "Cross-layer optimization for streaming scalable video over fading wireless networks," IEEE J. Sel. Areas Commun., vol. 28, no. 3, pp. 344-353, Apr. 2010.

15] E. Piri, M. Uitto, J. Vehkapera, and T. Sutinen, "Dynamic cross-layer adaptation of scalable video in wireless networking," in Proc. IEEE GLOBECOM, Dec. 2010, pp. 1-5.
[16] R. Radhakrishnan and A. Nayak, "Cross layer design for efficient video 950 streaming over LTE using scalable video coding," in Proc. IEEE ICC, 951 Jun. 2012, pp. 6509-6513.

[17] Video Trace Library. [Online]. Available: http://trace.eas.asu.edu/ 953

[18] C. Chen, R. W. Heath Jr., A. C. Bovik, and G. D. Veciana, "A Markov 954 decision model for adaptive scheduling of stored scalable videos," IEEE 955 Trans. Circuit Syst. Video Technol., vol. 23, pp. 1081-1095, Jun. 2013. 956

[19] A. Dembo, and O. Zeitouni, Large Deviations Techniques and Applica- 957 tions. 2nd ed. Berlin, Germany: Springer-Verlag, 1998.

958

[20] Apple HLS, "HTTP Live Streaming draft-pantos-http-live-streaming-08 959 (IETF draft)."

[21] Third-Generation Partnership Project (3GPP), “Transparent end-to-end 961 Packet Switched Streaming Service (PSS); protocols and codecs," ETSI, 962 Sophia-Antipolis, France, 3GPP TS 26.234, 2010.

[22] C. Hsu, and M. Hefeeda, "Partitioning of multiple fine-grained scalable 964 video sequences concurrently streamed to heterogeneous clients," IEEE 965 Trans. Multimedia, vol. 10, no. 3, pp. 457-469, Apr. 2008.

[23] Z. Chen, M. Li, and Y. Tan, "Perception-aware multiple scalable video 967 streaming over WLANs," IEEE Signal Process. Lett., vol. 17, no. 7, 968 pp. 675-678, Jul. 2010.

[24] Third-Generation Partnership Project (3GPP), “Transparent end-to-end 970 Packet Switched Streaming Service (PSS); progressive download and 971 dynamic adaptive streaming over HTTP (3G)," ETSI, Sophia-Antipolis, 972 France, 3GPP TS 26.247, 2011.

[25] Information Technology-Dynamic Adaptive Streaming Over HTTP 974 (DASH)_Part 1: Media Presentation Description and Segment Formats, 975 ISO/IEC 23009-1:2012, 2012,

[26] G. Tian, and Y. Liu, "Towards agile and smooth video adaptation 977 in dynamic HTTP streaming," in Proc. ACM CoNEXT, Dec. 2012, 978 pp. 109-120.

[27] J. Jiang, V. Sekar, and H. Zhang, "Improving fairness, efficiency, and 980 stability in HTTP-based adaptive video streaming with FESTIVE," in 981 Proc. ACM CoNEXT, Dec. 2012, pp. 97-108.

[28] J. Chen, R. Mahindra, M. A. Khojastepour, S. Rangarajan, and 983 M. Chiang, "A scheduling framework for adaptive video delivery over 984 cellular networks," Proc. ACM MOBICOM, Sep. 2013, pp. 389-400. 985

[29] J. Little, "A proof of the queuing formula: $L=\lambda W$," Oper. Res., vol. 9, 986 no. 3, pp. 383-387, May 1961.

[30] A. Wilig, "A Short Introduction to Queueing Theory," 1999. [Online]. 988 Available: http://www.cs.ucf.edu/lboloni/Teaching/EEL6785_Fall2010/ 989 slides/QueueingTheory.pdf

[31] F. Ishizaki, and F. Takine, "Loss probability in a finite discrete-time queue 991 in terms of the steady state distribution of an infinite queue," Queue Syst., 992 vol. 31, no. 3/4, pp. 317-326, Jul. 1999.

[32] N. B. Shroff and M. Schwartz, "Improved loss calculations at an ATM 994 multiplexer," IEEE/ACM Trans. Netw., vol. 6, no. 4, pp. 411-421, 995 Aug. 1998

996

[33] H. S. Kim and N. B. Shroff, "Loss probability calculations and asymp- 997 totic analysis for finite buffer multiplexers," IEEE/ACM Trans. Netw., 998 vol. 9, no. 6, pp. 755-768, Dec. 2001.

[34] H. Bobarshad, M. van der Schaar, A. H. Aghvami, R. S. Dilmaghani, 1000 and M. R. Shikh-Bahaei, "Analytical modeling for delay-sensitive video 1001 over WLAN," IEEE Trans. Multimedia, vol. 14, no. 2, pp. 401-414, 1002 Apr. 2012.

[35] S. T. Cheng, M. H. Tao, and C. Y. Wang, "Adaptive channel switching for 1004 centralized MAC protocols in multihop wireless networks," IEEE Trans. 1005 Commun., vol. 58, no. 1, pp. 228-234, Jan. 2010.

[36] H. Bobarshad and M. Shikh-Bahaei, "M/M/1 queuing model for adap- 1007 tive cross-layer error protection in WLANs," in Proc. IEEE WCNC, 1008 Nov. 2009, pp. 1-6.

[37] Y. Xu et al., "Probabilistic analysis of buffer starvation in Markovian 1010 queues," in Proc. IEEE INFOCOM, Mar. 2012, pp. 1826-1834. 1011

[38] M. Mandjes, Large Deviations for Gaussian Queues: Modelling Commu- 1012 nications Networks. Hoboken, NJ, USA: Wiley, 2007.

[39] G. E. P. Box, G. M. Jenkins, and G. C. Reinsel, Time Series Analysis 1014 Forecasting and Control. Englewood Cliffs, NJ, USA: Prentice-Hall, 1015 1994.

1016

[40] Motorola and Nokia, "Revised CQI proposal," 3GPP RAN WG1, Sophia, 1017 Antipolis, France, Tech. Rep. R1-02-0675, Apr. 2002.

1018

[41] P. Seeling and M. Reisslein, "Video transport evaluation with H.264 1019 video traces," IEEE Commun. Surveys Tuts., vol. 14, no. 4, 4th Quart. 1020 2012.

[42] Gabin et al., "3GPP mobile multimedia streaming stands," IEEE Signal 1022 Process. Mag., vol. 27, no. 6, pp. 134-138, Nov. 2010.

[43] C. H. Lin, C. H. Ke, C. K. Shieh, and N. K. Chilamkurti, "The packet loss 1024 effect on MPEG video transmission in wireless networks," in Proc. IEEE 1025 AINA, Apr. 2006, pp. 565-572. 


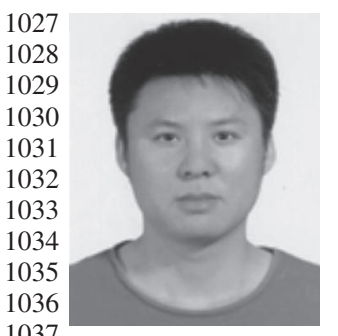

1037

1038 future networks.

1039 Dr. Yang received the Lu Jia-Xi Young Talent Award from the Chinese 1040 Academy of Sciences in 2009.

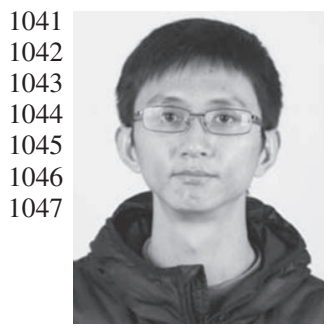

Yongyi Ran received the B.S. and Ph.D. degrees in 2008 and 2014, respectively, from the University of Science and Technology of China, Hefei, China, where he is currently a Postdoctoral Researcher.

His research interests include cloud computing, service management, future networks, and stochastic optimization.

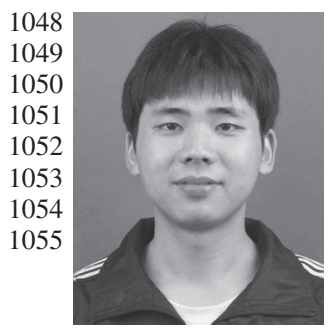

Shuangwu Chen received the B.S. degree in 2011 from the University of Science and Technology of China, Hefei, China, where he is currently working toward the Ph.D. degree with the School of Information Science and Technology.

His research interests include multimedia communications, future networks, and stochastic optimization.

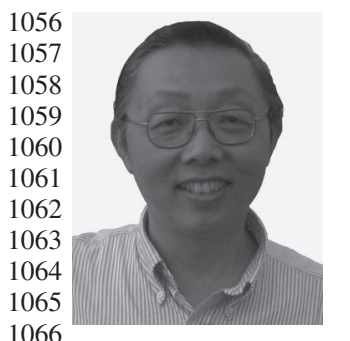

Weiping Li (F'00) received the B.S. degree from the University of Science and Technology of China (USTC), Hefei, China, in 1982 and the M.S. and Ph.D. degrees from Stanford University, Stanford, CA, USA, in 1983 and 1988, respectively, all in electrical engineering.

In 1987, he joined the Faculty of Lehigh University, Bethlehem, PA, USA, as an Assistant Professor with the Department of Electrical Engineering and Computer Science. In 1993, he was promoted to Associate Professor with Tenure. In 1998, he was 1067 promoted to Full Professor. From 1998 to 2010, he was with several high1068 technology companies in the Silicon Valley, where he had technical and 1069 management responsibilities. In March 2010, he returned to the USTC and is 1070 currently a Professor with the School of Information Science and Technology. 1071 Prof. Li was elected Fellow of IEEE for contributions to image and video 1072 coding algorithms, standards, and implementations. He served as a member 1073 of Moving Picture Experts Group (MPEG) of the International Organization 1074 for Standardization (ISO) and an Editor of MPEG-4 International Standard. 1075 He served as a Founding Member of the Board of Directors of the MPEG-4 1076 Industry Forum. His inventions on fine granularity scalable video coding and 1077 shape adaptive wavelet coding have been included in the MPEG-4 International 1078 Standard. As a Technical Adviser, he also made contributions to the Chinese 1079 Audio Video Coding Standard and its applications. He served as the Chair of 1080 several technical committees within the IEEE Circuits and Systems Society 1081 and at IEEE international conferences. He served as the Chair of the Best 1082 Student Paper Award Committee for the SPIE Visual Communications and 1083 Image Processing Conference. He served as the Editor-in-Chief of the IEEE 1084 TRANSACTIONS ON CiRCUITS AND SYSTEMS FOR VIDEO TECHNOLOGY. $1085 \mathrm{He}$ served as a Guest Editor for a special issue of the PROCEEDINGS OF THE 1086 IEEE. He has made many contributions to international standards. He received 1087 the Certificate of Appreciation from ISO and the International Electrotechnical 1088 Commission as a Project Editor in the development of International Standard 1089 in 2004, the Spira Award for Excellence in Teaching in 1992 from Lehigh 1090 University, and the first Guo Moruo Prize for Outstanding Student in 1980 from 1091 the USTC.

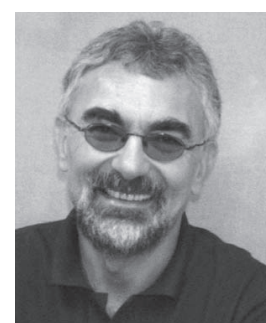

Lajos Hanzo (M'91-SM'92-F'04) received the 1092 Master's degree in electronics, the Ph.D. degree, and 1093 the Doctor Honoris Causa degree from the Technical 1094 University of Budapest, Budapest, Hungary, in 1976, 1095 1983, and 2009 respectively, and the D.Sc. degree 1096 from the University of Southampton, Southampton, 1097 U.K., in 2004.

1098

During his career in telecommunications, he 1099 has held various research and academic posts in 1100 Hungary, Germany, and the U.K. Since 1986, he has 1101 been with the School of Electronics and Computer 1102 Science, University of Southampton, Southampton, U.K., where he holds 1103 the Chair in telecommunications. He was a Chaired Professor of Tsinghua 1104 University, Beijing, China. He is the coauthor of 20 John Wiley/IEEE Press 1105 books on mobile radio communications, totalling in excess of 10000 pages, and 1106 has published more than 1400 research entries on IEEE Xplore. He is currently 1107 directing an academic research team, working on a range of research projects 1108 in the field of wireless multimedia communications sponsored by industry, 1109 the Engineering and Physical Sciences Research Council (EPSRC) U.K., the 1110 European IST Program, and the Mobile Virtual Centre of Excellence, U.K. He 1111 is an enthusiastic supporter of industrial and academic liaison, and he offers a 1112 wide range of industrial courses.

Dr. Hanzo has acted as a Technical Program Committee Chair for IEEE con- 1114 ferences, presented keynote lectures, and has received a number of distinctions. 1115 $\mathrm{He}$ is the Governor of the IEEE Vehicular Technology Society and the Past 1116 Editor-in-Chief of the IEEE Press. 


\section{AUTHOR QUERIES}

\section{AUTHOR PLEASE ANSWER ALL QUERIES}

AQ1 = Please check if "Star War IV" should be "Shawshank Redemption"

AQ2 = Please check if changes made in this sentence are appropriate.

AQ3 = Provided URL in Ref. [30] was not found. Please check.

\section{END OF ALL QUERIES}




\title{
Online Source Rate Control for Adaptive Video Streaming Over HSPA and LTE-Style Variable Bit Rate Downlink Channels
}

\author{
4 Jian Yang, Member, IEEE, Yongyi Ran, Shuangwu Chen, Weiping Li, Fellow, IEEE, and Lajos Hanzo, Fellow, IEEE
}

\begin{abstract}
5 Abstract-Online source rate control (RC) is designed for video 6 streaming over high-speed packet access (HSPA) and Long-Term 7 Evolution (LTE)-style variable bit rate (VBR) downlink channels. 8 The problem is formulated as the adaptive adjustment of the 9 operational mode of a video encoder based on the buffer overflow 10 probability (BOP) feedback received from the radio link control 11 (RLC) layer at the base station (BS). This allows us to maximize 12 the attainable visual quality while keeping the transmitter BOP 13 below a desired threshold and maintaining a video delay as low as 14 possible. We derive an online measurement-based BOP estimation 15 model for the RLC buffer, which is capable of operating with 16 no prior knowledge of the channel variations and of the video 17 characteristics. Based on this estimation model, an online adaptive $18 \mathbf{R C}$ algorithm is proposed to seamlessly adapt the bit stream to 19 the characteristics of VBR channels. Our experiments are con20 ducted in multiuser scenarios using VBR video encoding combined 21 with adaptive modulation and coding (AMC) in the transceiver. 22 The results demonstrate that the proposed source $\mathrm{RC}$ regime 23 supports near-instantaneous yet smooth bit stream adaptability, 24 which makes it useful for HSPA and LTE-style systems for the 25 sake of accommodating unknown video traffic characteristics and 26 dynamically fluctuating propagation conditions.
\end{abstract}

28 Index Terms-Large deviation principle (LDP), rate control (RC), variable bit rate (VBR) channel.

\section{INTRODUCTION}

30 DVANCED transceiver techniques, including adaptive 32 modulation and coding (AMC) and hybrid automatic re33 peat request (HARQ), are widely applied in wireless systems to 34 combat the effects of both fading and interference [1], [2]. For 35 instance, both the third-generation $(3 \mathrm{G})$ and high-speed packet

Manuscript received January 10, 2014; revised July 21, 2014 and November 3, 2014; accepted January 24, 2015. This work was supported in part by the National Natural Science Foundation of China under Grant 61174062; by the State Key Program of the National Natural Science Foundation of China under Grant 61233003; and by the Fundamental Research Funds for the Central Universities. The work of L. Hanzo was supported by the European Research Council under an Advanced Fellow Grant. The review of this paper was coordinated by Prof. N. Arumugam.

J. Yang, Y. Ran, S. Chen, and W. Li are with the School of Information Science and Technology, University of Science and Technology of China, Hefei 230026, China (e-mail: jianyang@ ustc.edu.cn; yyran@ mail.ustc.edu.cn; chensw@mail.ustc.edu.cn; wpli@ustc.edu.cn).

L. Hanzo is with the School of Electronics and Computer Science, University of Southampton, Southampton SO17 1BJ, U.K. (e-mail: 1h@ecs.soton.ac.uk).

Color versions of one or more of the figures in this paper are available online at http://ieeexplore.ieee.org.

Digital Object Identifier 10.1109/TVT.2015.2398515 access (HSPA) apply AMC and HARQ in the physical layer 36 relying on the channel quality indicator's (CQI) feedback from 37 the user equipment (UE) to achieve prompt link adaptation. The 38 Long-Term Evolution (LTE) Advanced and WiMax standards 39 also provide similar link adaptation based on AMC and short 40 time-slot duration periods. Naturally, agile link adaptation may 41 result in a time-varying effective throughput of the channel. 42 Therefore, supporting near-instantaneous bit rate adaptivity 43 is one of the most important features of video streaming 44 applications.

Rate control (RC) aims to control the video bit rate to match 46 the channel's achievable bit rate, given the prevalent channel 47 quality to keep the video quality as high as possible [3]. Most 48 existing studies of RC focus on allocating a bit rate budget to 49 each group of pictures (GOP), frames, or macroblocks at the 50 encoder. For instance, an efficient RC scheme was designed 51 for MPEG-4 based on a quadratic rate-distortion (R-D) model 52 invoked to maintain the target bit rate in [4]. In [5], the quadratic 53 $\mathrm{R}-\mathrm{D}$ model was also adopted to derive an adaptive $\mathrm{RC}$ for 54 H.264 to meet the target bit rate. In [6], RC was designed 55 for scalable H.264/Advanced Video Coding (AVC) encoding. 56 H.264 reference software JM [7] has implemented the JVT- 57 G012 [8] RC algorithm, which relies on a combination of the 58 available channel bandwidth, the frame rate, the target buffer 59 level, and the actual buffer fullness for determining the number 60 of bits allocated for the current frame. A range of AMC-based 61 schemes were conceived in [9] where no extra buffering was 62 used by the RC. The design philosophy was to instruct the 63 video encoder to produce the exact number of bits for each 64 video frame, which was affordable for the near-instantaneous 65 HSPA-style AMC transceiver mode that was periodically sig- 66 naled back from the receiver to the transmitter. However, the 67 aforementioned RC schemes rely on the knowledge of the target 68 bit rate, which again requires the feedback of the expected bit 69 rate based on the estimated channel quality. Unfortunately, the 70 channel's bit rate fluctuations are not known a priori at the 71 transmitter and the video encoder. Therefore, these RC schemes 72 may impose substantial video quality fluctuations.

Recently, a control-theoretic approach-based RC regime has 74 been proposed in [10] by jointly considering the encoder's RC 75 and network congestion control. More specifically, an empirical 76 R-D source model, a channel-induced distortion model, and 77 their linearized models were applied to formulate a mathe- 78 matically tractable system model. However, it is a challenge 79 to formulate accurate models when streaming videos over 80 
81 time-varying channels. Typically, intelligent packet scheduling 82 is used at the base station (BS) by most of the existing solutions 83 to achieve channel-quality-dependent adaptive video streaming 84 over wireless networks. Cross-layer-adaptive scalable video 85 streaming has been proposed by informing the media access 86 control (MAC) layer of the amount of payload and the index of 87 the modulation-and-encoding scheme to improve the attainable 88 video quality [11]. An adaptive scalable video streaming strat89 egy relaying on controlling the MAC buffer was presented in 90 [12]. Both the packet deadlines and the channel characteristics 91 were considered in video packet scheduling at the BS [13]. 92 The cross-layer design-aided transmission of scalable video 93 streams [14] involves packet scheduling combined with a video 94 frame dropping strategy at the BS. Most of these papers discuss 95 the channel-dependent adaptation of the MAC layer of the BS 96 where no signaling is fed back to instruct the video source to 97 adjust its bit rate. Dropping packets at the BS is a waste of 98 resources both in the backhaul router and the core network. It 99 was shown in [15] that packet dropping at the video source is 100 more efficient than MAC layer dropping at the BS when we 101 have to reduce the congestion in both the wired core network 102 and in the wireless medium to the UE. By translating the 103 CQI values to the number of enhancement layers scheduled 104 for transmission, the video server facilitates adaptive video 105 streaming [16]. However, the link throughput at the BS is 106 determined not only by the CQI value but also by the MAC 107 layer scheduling strategy of a multiuser scenario. Moreover, 108 the bit rate of video clips having different amounts of motion 109 activity may be very different even if they have the same num110 ber of enhancement layers [17]. Therefore, such CQI-mapping111 based video source adaptation lacks robustness against the 112 time-variant number of users and against the heterogeneous 113 video bit rates. In [18], although a Markov decision process is 114 invoked for dynamic video scheduling, its offline computation 115 requires a priori knowledge of the channel dynamics. Hence, 116 a heuristic online scheduling algorithm is derived based on the 117 average channel throughput estimated with the aid of a first118 order autoregressive model [AR(1)]. In the HSPA/LTE system, 119 using a CQI-based link adaptation mechanism may lead to 120 a burst-by-burst transmission block adaptation, and the linear 121 estimator based AR(1) may not be appropriate for estimating 122 the average channel throughput.

123 Against this background, we proposed a novel online source 124 RC framework, where a buffer overflow probability (BOP) 125 estimator is employed at the radio link control (RLC) layer 126 of the BS, and the estimated BOP is signaled back to the 127 video source to control its bit rate. The motivation of using a 128 BOP-based feedback is that the BOP metric characterizes the 129 degree of matching between the video bit rate of the source 130 and the link throughput. Since the BOP is estimated before 131 a buffer overflow is encountered, its feedback may assist the 132 video source in promptly controlling the bit rate. In contrast to 133 the methods found in the open literature [9]-[14], [16], [18], the 134 main contributions of this paper are threefold.

- To enable the video encoder to generate a video stream that may be reliably delivered over variable bit rate (VBR) downlink channels, we formulate a constrained optimization problem subject to a constraint imposed on 138 the transmission BOP to guarantee a low delay.

- A BOP estimation model based on large deviation prin- 140 ciples (LDPs) [19] is proposed by monitoring the buffer 141 fullness and its variation at the BS's RLC layer. The 142 reason for applying the LDPs is because it accurately 143 characterizes the probability of rare events, which assists 144 us in achieving fine adjustment of the video source rate. 145

- We conceive an iterative RC algorithm to approach the 146 most beneficial encoder rate, thus circumventing the dif- 147 ficulty of directly solving the related constrained opti- 148 mization problem. The proposed RC algorithm allows the 149 encoder to adjust its bit rate to that of the VBR channel 150 without any a priori knowledge of both the channel 151 quality variations and of the characteristics of the video 152 source.

The remainder of this paper is organized as follows. 154 Section II describes the system model, including the formu- 155 lation of the source RC problem of video streaming over a 156 VBR downlink channel. In Section III, we apply the LDP in 157 [19] to derive a BOP estimation model, whereas an online 158 measurement-based RC is proposed in Section IV. Our numeri- 159 cal simulation results are presented in Section V to characterize 160 the attainable performance of the proposed algorithm. Finally, 161 our conclusions are offered in Section VI.

\section{SySTEM Model}

\section{A. System Overview}

Fig. 1 shows a typical video streaming scenario over a 166 wireless network. The main associated protocol stacks are also 167 shown in the lower part of the figure. Naturally, a video stream- 168 ing service involves not only maintaining the wireless connec- 169 tion between the mobile station (MS) and the base transceiver 170 station (BTS) but supporting the public network connection 171 (Internet) as well. The wired network provides high capacity 172 and stability; hence, video streaming over the wired network 173 has become a well-established service and has many successful 174 applications, including video conferencing, surveillance sys- 175 tems, and Internet Protocol (IP) television. By contrast, video 176 streaming over wireless networks faces unique challenges due 177 to the time-varying nature of the wireless channel and owing to 178 the scarcity of the system resources, which makes it difficult to 179 guarantee any specific video quality of service (QoS). Hence, 180 the wireless transmission in the video streaming service is likely 181 to be a bottleneck, which is the focus of this paper.

Fig. 2 shows the basic video processing in the video network 183 abstract layer (NAL) units of the 3GPP framework. A NAL 184 unit may be encapsulated in a Real-Time Transport Protocol 185 (RTP) data unit, and then, it may be transmitted over User 186 Datagram Protocol (UDP)/IP. HTTP-based streaming protocols 187 such as Apple HTTP Live Streaming (HLS) [20] are alternative 188 media streaming communication protocols, which are capable 189 of traversing any firewall or proxy server that lets through 190 standard HTTP traffic, unlike UDP-based protocols such as 191 RTP. 3GPP standardized an adaptive HTTP streaming protocol 192 


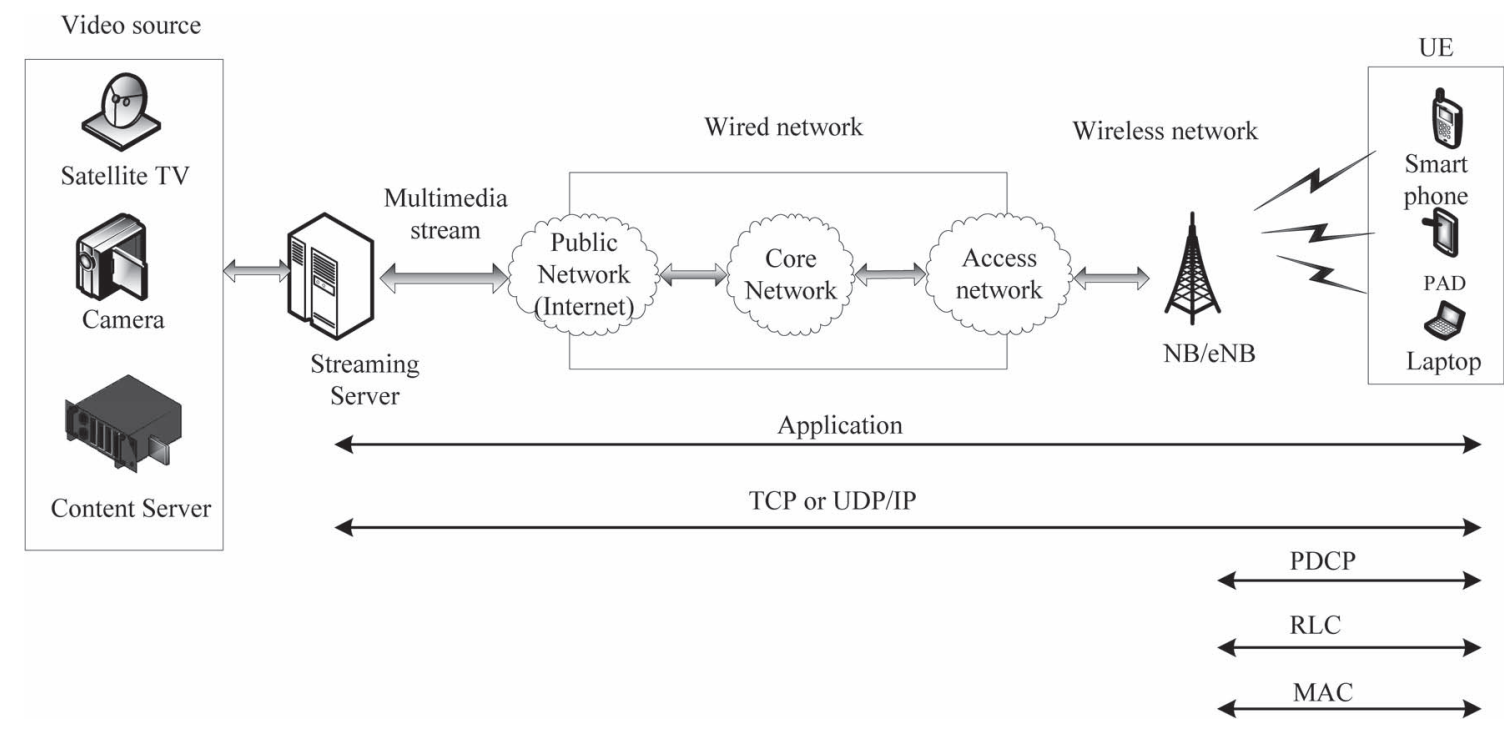

Fig. 1. Typical scenario for wireless video streaming in 3G Partnership Project (3GPP) framework.

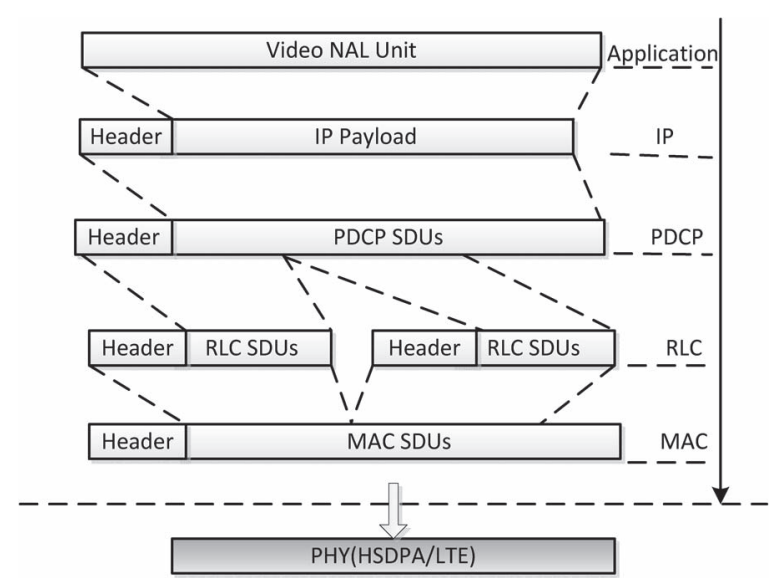

Fig. 2. Video data packetization at different layers in the 3GPP framework.

193 in Rel-9 [21]. Subsequently, we concentrate on the terminology 194 used in HSPA NB/eNB, as shown in Fig. 2. The IP/UDP/RTP 195 or IP/Transmission Control Protocol (TCP)/HTTP packet gen196 erated is encapsulated into a single Packet Data Convergence 197 Protocol (PDCP) packet that becomes an RLC service data 198 unit (SDU). Since a typical RLC SDU has a larger size than 199 an RLC protocol data unit (PDU), it has to be segmented into 200 smaller units. The length of the RLC PDU depends both on the 201 selected bearer and on the AMC mode used. The RLC PDUs are 202 forwarded to the MAC layer and are then encapsulated as MAC 203 PDUs. The main functions of the PDCP layer are robust header 204 compression and decompression, ciphering and deciphering, 205 the transfer of data, and PDCP sequence number maintenance. 206 The RLC layer in wireless systems is capable of operating 207 in both an unacknowledged mode (UM) and acknowledged 208 mode (AM), and both are capable of providing RLC PDU 209 loss detection. However, while the UM is unidirectional and 210 data delivery is not guaranteed, in the AM, automatic repeat 211 request (ARQ) is applied for reliable data transmission. The 212 main functions of the RLC layer include the transfer of upper layer PDUs; error correction relying on ARQ (only for AM); 213 and the concatenation, segmentation, and reassembly of RLC 214 SDUs, whereas the main functions of the MAC layer are 215 resource scheduling and multiplexing/demultiplexing of MAC 216 SDUs belonging to one or several logical channels into/from the 217 relevant transport blocks (TBs). By comparing the functions of 218 the RLC layer with those of the PDCP and MAC, the RLC is an 219 appropriate layer for us to construct a model for characterizing 220 the degree of matching between the network's throughput and 221 the video source bit rate. Generally, the detection of a lost 222 RLC PDU results in the loss of an entire PDCP packet; hence, 223 the encapsulated IP and the NAL unit are lost. From the 224 perspective of reacting to both the dynamics of the statistical 225 fluctuation of the teletraffic and the variable channel conditions, 226 agile bit rate adaptivity is one of the most important features 227 for seamless video streaming over wireless systems. There 228 are several ways of achieving bit rate adaptivity. For online 229 encoding applications, the bit rate adaptivity can be achieved 230 by controlling encoding parameters. For instance, H.264/AVC 231 supports these features mainly by dynamically varying the 232 quantizers but also by controlling temporal resolution. Scalable 233 Video Coding (SVC) is another technique of implementing 234 the bit rate adaptivity. It encodes the raw video clip into a 235 base layer and a number of enhancement layers with different 236 priorities. Naturally, the base layer has the highest priority since 237 it contains the video bits with the highest importance, which can 238 provide a minimum video quality. The enhancement layers with 239 lower priorities may be progressively encoded to further refine 240 the quality of the base-layer stream. This layered approach 241 of the SVC codec allows an encoded stream to be flexibly 242 prepared for meeting the bit rate constraint. In [22], the base- 243 layer rate for a given video sequence is optimized to achieve the 244 highest possible average perceived quality for heterogeneous 245 clients, whereas in [23], a distribution regime was conceived 246 for scalable videos, which guaranteed fairness for all end users. 247 In Dynamic Adaptive Streaming over HTTP (DASH), the video 248 content is partitioned into a sequence of small HTTP-based file 249 


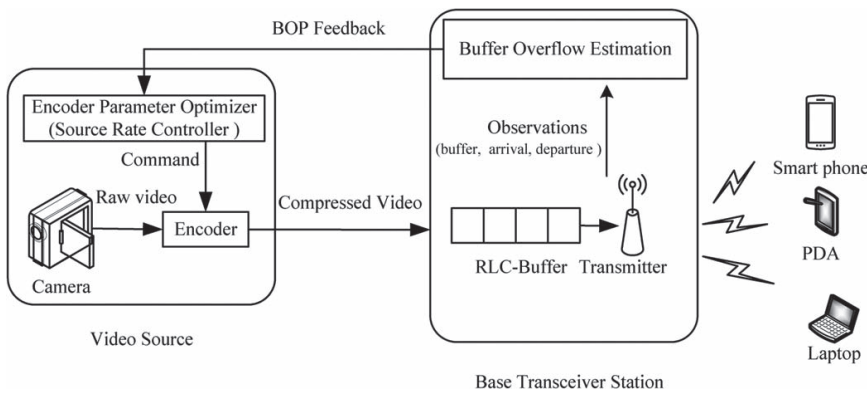

Fig. 3. Adaptive source RC framework for online video encoding.

250 segments, which are made available at a variety of different bit 251 rates. More explicitly, segments encoded at different bit rates 252 covering appropriately aligned short intervals of playback time 253 are made available. Naturally, DASH also provides a flexible 254 way of adjusting the video source bit rate. 3GPP has defined its 255 Rel-10 version of DASH, termed as 3GP-DASH [24], which 256 is a profile compatible with MPEG-DASH [25]. More work 257 on HTTP-based adaptive video streaming can be found in 258 [26]-[28].

259 Since the RLC buffer fullness indicates the degree of match260 ing between the source video bit rate and the channel bit rate, 261 we subsequently design a buffer fullness estimation scheme 262 where the buffer fullness estimated at the RLC layer is fed 263 back to the video source to adapt its bit rate. Without loss 264 of generality, we consider a specific scenario of the online 265 encoding $\mathrm{RC}$ relying on controlling encoding parameters, i.e., 266 dynamically changing the quantization parameters (QPs). It 267 is straightforward to extend the proposed method to both 268 SVC streaming and to HTTP-DASH scenarios, as discussed in 269 Section II-C.

\section{B. Problem Formulation}

271 The block diagram of a wireless video streaming system 272 equipped with an encoder parameter optimizer conceived to 273 control the source rate is shown in Fig. 3, which relies on a 274 camera, a video encoder, and a BTS. The camera samples the 275 video scene at certain frame scanning and forwards the frames 276 to the encoder, which forwards the compressed video to the 277 transmitter's buffer at the RLC layer for transmission. Here, we 278 assume that the channel between the video source and the BTS 279 is wired and reliable. The source RC problem is eliminating the 280 congestion in the BTS while satisfying the delay constraints. 281 Our basic philosophy is to feed back the mismatch between the 282 current source rate and the channel's affordable throughput to 283 the encoder parameter optimizer to adapt the source rate. If a 284 mismatch does occur, the encoder parameter optimizer sends 285 a command to the video encoder to adjust the video source 286 rate. To quantitatively characterize this mismatch, we define 287 BOP as the probability that the current wireless channel quality 288 provides an insufficient throughput for the current video bit rate. 289 A sufficiently low BOP indicates that we may increase the video 290 bit rate for transmission over the wireless channel to achieve a 291 higher video quality.

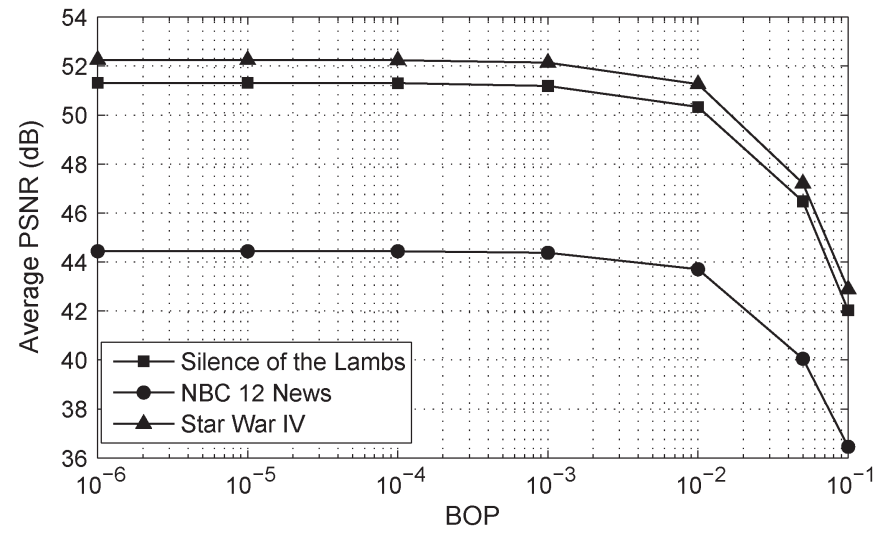

Fig. 4. Relation between BOP and received video quality by using three different video clips, namely, the Silence of the Lambs, NBC 12 News, and Star Wars IV.

Let $\mathcal{M} \triangleq\left\{M_{1}, \ldots, M_{L}\right\}$ be the operational mode set of the 292 video encoder. The video bit rate corresponding to the opera- 293 tional mode $M_{i}(i=1, \ldots, L)$ is denoted by $r_{i}$. Let us assume 294 that $r_{1}<r_{2}<\cdots<r_{L}$, which implies that a higher opera- 295 tional mode results in a higher video bit rate and hence achieves 296 a better video quality. The operational mode is controlled by the 297 encoder parameters. For instance, we can control the encoding 298 mode by appropriately setting the I-P-B quantization mode or 299 the target video bit rate parameter of the JM encoder. Here, 300 we consider adapting the source rate to the channel quality by 301 dynamically adjusting the operational mode (i.e., the encoding 302 parameters) of the video encoder.

303

We used transmission slots of equal duration, which are nor- 304 malized to unity. The RLC SDUs, which contain the video data 305 generated by the video encoder, arrive at the RLC buffer, and 306 they are then queued until they are transmitted. Since the data 307 are processed at packet level instead of bit granularity, we define 308 the length of the RLC buffer as the number of RLC SDUs. Let 309 $A_{n} \in \mathcal{A} \triangleq\{0, \ldots, A\}$ denote the number of RLC SDU arrivals 310 in slot $n$, where $A$ is the maximum number of RLC SDU ar- 311 rivals in a single slot, whereas $D_{n} \in \mathcal{D} \triangleq\{0, \ldots, D\}$ is defined 312 as the number of RLC SDUs transmitted in slot $n$, where $D$ is 313 the maximum number of RLS SDUs transmitted in a single slot. 314 It should be noted that an agile and sophisticated link adaptation 315 mechanism based on AMC and HARQ is applied to maintain 316 the required target bit error rate in most state-of-the-art wireless 317 communication systems such as high-speed downlink packet 318 access, 3G LTE, and WiMAX. Both AMC and HARQ rely on 319 the CQI feedback received from the mobile terminals, which 320 results in a burst-by-burst adaptive channel throughput. Hence, 321 the downlink packet departure process $D_{n}$ is assumed to be 322 an independent identically distributed (i.i.d.) sequence. Let $Q_{n} 323$ denote the buffer fullness expressed in terms of the number of 324 packets at the end of slot $n$. The dynamics of the buffer fullness 325 may be described as

$$
Q_{n}=\max \left\{Q_{(n-1)}-D_{n}, 0\right\}+A_{n} .
$$

According to Little's theorem [29], we have

$$
\bar{Q}=\lambda \bar{D}
$$


DASH Switching Command

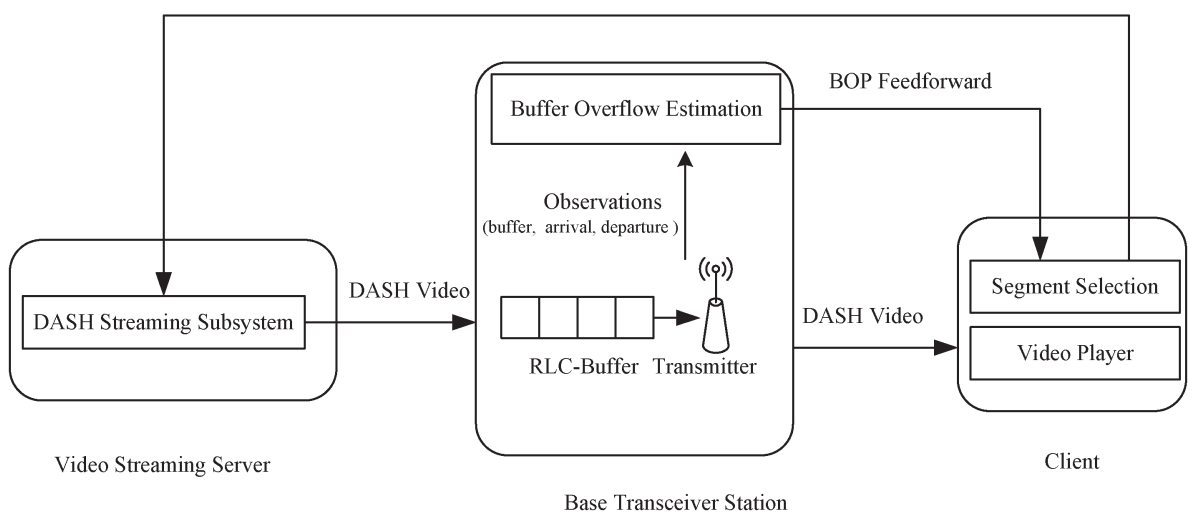

Fig. 5. Adaptive source RC framework for the DASH streaming application.

328 where $\bar{Q}$ is the average buffer fullness, $\bar{D}$ is the average delay, 329 and $\lambda$ is the packet arrival rate. This implies that, if $\lambda$ is given, 330 having a higher buffer fullness value implies a higher delay. 331 Hence, we treat the delay as being synonymous with buffer 332 fullness. Our objective is to select the best encoding mode for 333 controlling the buffer fullness to satisfy the delay constraint 334 of the specific video application. Let us formally define the $335 \mathrm{BOP}$ as

$$
p_{o}=P\left(Q>B_{h}\right)
$$

336 where $Q$ is the buffer fullness and $B_{h}$ is the buffer threshold. 337 Higher BOP implies higher buffer fullness in the near future, 338 in turn inducing a higher delay. To guarantee lip-synchronized 339 interactive low-delay video transmission, the BOP should be 340 kept low. Hence, the problem of RC may be reinterpreted as the 341 problem of selecting the best encoding mode subject to a given 342 BOP, which can be formulated as

$$
\begin{array}{ll}
\max _{m \in \mathcal{M}} & m \\
\text { s.t. } & P\left(Q^{m}>B_{h}\right)<p_{\text {qos }}
\end{array}
$$

343 where $Q^{m}$ denotes the buffer fullness corresponding to the video 344 encoding mode $m$, and $p_{\text {qos }}$ is the maximum tolerable BOP. 345 It should be noted that the queue length $P(Q>x)$ in the 346 scenario of an infinite buffer system is called an overflow 347 probability or a tail probability [30], which has been used 348 as a performance metric for an infinite buffer-aided system 349 [31]-[33]. However, practical systems have a limited buffer 350 capacity. When a video frame arrives at a full buffer, it gets 351 dropped. Hence, the packet loss probability (PLP) $P_{L}\left(B_{h}\right)$ 352 is invoked to characterize the performance of a finite-buffer353 based system having a capacity $B_{h}$ of video frames. For video 354 streaming services, the PLP is an important QoS measure, but 355 it is difficult to directly estimate the PLP of a general finite356 buffer-based system. Fortunately, a theoretical justification was 357 provided to approximate the PLP of a finite-buffer-based sys358 tem with the aid of estimating the BOP of its infinite buffer 359 counterpart [33]. Hence, we treat the BOP as being identical to 360 the PLP, which implies that the video source rate optimization 361 problem of a finite-buffer-based system having a capacity $B_{h}$ of frames can be mapped to the mathematical model of (4) and 362 (5). We have confirmed, by our simulation studies, the plausible 363 fact that a high BOP may severely degrade the QoS, which is 364 quantitatively characterized in Fig. 4 and motivates us to use 365 the BOP for the feedback control.

\section{Extensions for SVC and DASH Scenarios}

The scheme shown in Fig. 3 is a point-to-point representation 368 of our proposed framework, which may be also used for stream- 369 ing to multiple users, as shown in our forthcoming SVC/HTTP- 370 DASH streaming applications.

The framework detailed in Section II-B may be readily 372 extended to SVC and HTTP-DASH scenarios. For an SVC 373 scenario, a scalable video stream consists of a base layer 374 $l_{1}$ and $(L-1)$ enhancement layers, i.e., $\left\{l_{2}, l_{3}, \ldots, l_{L}\right\}$. The 375 operational mode $M_{i}(i=1, \ldots, L)$ is defined as the scenario 376 when the first $i$ layers are selected for transmission. Thus, the 377 source $\mathrm{RC}$ via adaptive enhancement layer selection can be also 378 formulated as the problem [see (4) and (5)]. The correspond- 379 ing video source in Fig. 3 may be also replaced by a video 380 streaming server, which maintains the sessions corresponding 381 to the multiple video users and receives the BOP feedback of 382 a specific session for adjusting the number of the transmitted 383 enhancement layers.

It should be noted that HTTP-DASH applied the pull-based 385 streaming paradigm rather than the traditional push-based 386 streaming paradigm relying on protocols such as the Real-Time 387 Streaming Protocol. The client plays the central role of driving 388 the video adaptation. To comply with the basic rule of DASH, 389 the framework for the DASH streaming applications shown 390 in Fig. 5 is based on the BOP feedforward to the client. The 391 client may then use the received BOP to select the future video 392 segments to be fetched. The DASH streaming server maintains 393 multiple sessions for the sake of supporting video streaming to 394 multiple users. In a DASH scenario, the operational mode set 395 $\mathcal{M}$ is defined as the adaptation set, where the operational mode 396 $M_{i}(i=1, \ldots, L)$ denotes streaming the chunks corresponding 397 to the $i$ th quality level. Then, the source RC via HTTP-DASH 398 can be similarly described as in (4) and (5) since a higher video 399 quality implies a higher bit rate. 
403 Naturally, the BOP estimation is a key step to successfully 404 solve the problem [see (4) and (5)]. To provide a high quality 405 of experience for a video streaming service, an occurrence of 406 buffer overflow is expected to be a rare event. The theory of 407 large deviation provides a useful way of accurately characteriz408 ing the probability of rare events. Therefore, we present a large 409 deviation-based BOP estimation model here. Although our 410 practical buffer is of finite size, the BOP of the corresponding 411 infinite-buffer-based system can be invoked to estimate the 412 PLP, as shown in [31]-[33]. Hence, we subsequently aim to 413 estimate the BOP of the corresponding infinite-buffer-based 414 system. To derive an analytical model, the BOP is estimated 415 based on the M/M/1 queueing model, which has been widely 416 applied in wireless networks [34]-[37]. The buffer at the RLC 417 is modeled as the M/M/1 queue, where $\lambda$ RLC SDUs/slot and $418 \mu \mathrm{RLC}$ SDUs/slot denote the Poissonian arrival rate and the

419 departure rate (or service rate), respectively. Let us now define 420 the large deviation theory in [19] for estimating the probability 421 of $P\left(Q_{n+N}>B_{h} \mid Q_{n}\right)$, where $Q_{n}$ is the buffer length during 422 the current time slot $n$. During the $k$ th slot, the evolution of 423 buffer length can be represented by

$$
I_{k}=A_{k}-\min \left(Q_{(k-1)}, D_{k}\right)
$$

424 Then, we have $Q_{n+N}=Q_{n}+\sum_{k=n+1}^{n+N} I_{k}$. Furthermore, $425 P\left(Q_{n+N}>B_{h} \mid Q_{n}\right)$ can be rewritten as

$$
\begin{aligned}
P\left(Q_{n+N}>B_{h} \mid Q_{n}\right) & =P\left(Q_{n}+\sum_{k=n+1}^{n+N} I_{k}>B_{h}\right) \\
& =P\left(\frac{1}{N} \sum_{k=n+1}^{n+N} I_{k}>a\right)
\end{aligned}
$$

426 where we have $a=\left(B_{h}-Q_{n}\right) / N$. Next, we apply the LDP to 427 the sequence $I_{n+1}, I_{n+2}, \ldots$ to derive $P\left((1 / n) \Sigma_{k=1}^{n} I_{k}>a\right)$. 428 The LDP can be briefly described as follows [38].

429 Definition: A sequence $X_{1}, X_{2}, \ldots$ obeys the LDP associ430 ated with rate function $I(\cdot)$ if the following conditions apply.

431 1) For any closed set $F$, we have

$$
\limsup _{N \rightarrow \infty} \frac{1}{N} \log P\left(\frac{1}{N} \sum_{i=1}^{N} X_{i} \in F\right) \leq-\inf _{a \in F} I(a) .
$$

2) For any open set $G$, we have

$$
\liminf _{N \rightarrow \infty} \frac{1}{N} \log P\left(\frac{1}{N} \sum_{i=1}^{N} X_{i} \in G\right) \geq-\inf _{a \in G} I(a)
$$

433 Obviously, $I_{k} \in \mathbb{R}(k \in[n+1, n+N])$ represents i.i.d. 434 random variables with a mean of $E\left[I_{k}\right]=\lambda-\mu$ and moment435 generating function (MGF) of $M(\theta)=E\left[e^{\theta I_{k}}\right]$ that is finite 436 in a neighborhood of 0. According to Cramér's theorem [38], $437 I_{n+1}, I_{n+2}, \ldots$ obeys the LDP associated with the rate function
$I(a)=\sup _{\theta>0}(\theta a-\log M(\theta))$, which implies that, for any 438 $a>\lambda-\mu$, we have

$$
\lim _{N \rightarrow \infty} \frac{1}{N} \log P\left(\frac{1}{N} \sum_{i=n+1}^{n+N} I_{i}>a\right)=-I(a) .
$$

It should be noted that (10) is logarithmically asymptotic. 440 Hence, when $N$ is large, the BOP can be approximated as

$$
P\left(\frac{1}{N} \sum_{i=n+1}^{n+N} I_{i}>a\right) \approx \exp [-N I(a)] .
$$

Since $A_{k}$ obeys Poisson's distribution, we have

$$
E\left[e^{\theta A_{k}}\right]=\sum_{\eta=0}^{\infty} e^{\theta \eta} \frac{\lambda^{\eta}}{\eta !} e^{-\lambda}=\exp \left(\lambda e^{\theta}-\lambda\right) .
$$

Similarly, we may arrive at $E\left[e^{-\theta D_{k}}\right]=\exp \left(\mu e^{-\theta}-\mu\right)$. Then, 443 we may derive the $\mathrm{MGF} M(\theta)$ as follows:

$$
\begin{aligned}
M(\theta) & =E\left[e^{\theta I_{k}}\right] \\
& =E\left[e^{\theta A_{k}}\right] E\left[e^{-\theta D_{k}}\right] \\
& =\exp \left(\lambda e^{\theta}+\mu e^{-\theta}-\lambda-\mu\right) .
\end{aligned}
$$

Hence, the corresponding rate function $I(a)$ can be rewritten as 445

$$
\begin{aligned}
I(a) & =\sup _{\theta>0}\left(\theta a-\lambda e^{\theta}-\mu e^{-\theta}+\lambda+\mu\right) \\
& =\left.\left(\theta a-\lambda e^{\theta}-\mu e^{-\theta}+\lambda+\mu\right)\right|_{\theta=\log \frac{a+\sqrt{a^{2}+4 \lambda \mu}}{2 \lambda}} \\
& =a \log \frac{a+\sqrt{a^{2}+4 \lambda \mu}}{2 \lambda}-\sqrt{a^{2}+4 \lambda \mu}+\lambda+\mu .
\end{aligned}
$$

If $\lambda$ and $\mu$ are given for any encoding mode $m \in \mathcal{M}$, the BOP 446 $P\left(Q_{n+N}>B_{h} \mid Q_{n}\right)$ can be estimated by using (11) and (14). 447 The problem [see (4) and (5)] can be then solved by calculating 448 the BOPs corresponding to all encoding modes in $\mathcal{M}$. However, 449 in practical situations, both $\lambda$ and $\mu$ depend on the network's 450 condition and on the source encoding mode, and they are not 451 based on prior knowledge for each encoding mode.

452

An attractive technique is applying an iterative policy to 453 solve the problem [see (4) and (5)]. Specifically, at the current 454 mode update period, we can calculate the BOP corresponding 455 to the current encoding mode by the online estimation of the 456 arrival rate $\lambda$ and the departure rate $\mu$. Although $\lambda$ and $\mu$ can 457 be estimated by counting the number of RLC SDU arrivals 458 and departures over a time period of $T$, they may change 459 over time due to time-varying network conditions and dynamic 460 encoding mode switching. The dynamic nature of $\lambda$ and $\mu$ may 461 be modeled using an autoregressive integrated moving average 462 (ARIMA) process [39] as follows:

463

$$
\begin{aligned}
& \left(1-\sum_{i=1}^{p} a_{i} D^{i}\right)(1-D)^{d_{1}} \lambda(t)=\left(1+\sum_{i=1}^{q} b_{i} D^{i}\right) \varepsilon(t) \\
& \left(1-\sum_{i=1}^{p} c_{i} D^{i}\right)(1-D)^{d_{2}} \mu(t)=\left(1+\sum_{i=1}^{q} d_{i} D^{i}\right) \mu(t)
\end{aligned}
$$


464 where $D$ is the unit time-delay operator, and $\varepsilon(t)$ is the estima465 tion error. Therefore, the task of estimating $\lambda$ and $\mu$ becomes 466 the task of estimating parameters $p, q, d_{1}, d_{2}, a_{i}, b_{i}, c_{i}$, and $d_{i}$ 467 for the ARIMA model [39]. Once $\lambda$ and $\mu$ have been estimated, 468 the corresponding BOP can be directly calculated by using (11) 469 and (14). If the BOP estimate fails to satisfy the constraint (5), 470 the source encoding rate should be decreased, thus reducing the 471 BOP. Otherwise, if the BOP is much lower than $p_{\text {qos }}$, the video 472 source bit rate may be increased, thus improving the video 473 quality. Applying this iterative encoding mode update, we can 474 iteratively solve the problem [see (4) and (5)] to find a new 475 optimal encoding mode.

476 It should be noted that the aforementioned method relies 477 on the classic M/M/1 queuing model, albeit the Poissonian 478 assumption may be not the most realistic packet arrival and 479 departure model. Moreover, our model of the BOP requires 480 estimating both $\lambda$ and $\mu$. In the next section, we propose an 481 online measurement-based RC, which no longer relies on the $482 \mathrm{M} / \mathrm{M} / 1$ queuing model and on the estimation of the RLC SDU 483 arrival rate $\lambda$ and the departure rate $\mu$.

\section{IV. Online Encoding Mode Switching-Based 485 RATE CONTROL FOR VIDEO STREAMING}

486 Let the index of the current time slot be $n$ and the current 487 buffer length be $Q_{n}^{m}$, whereas $m=M_{i}$ denotes the current 488 video encoding mode. We estimate the BOP at the $(n+N)$ th $489(N>0)$ slot under the assumption of maintaining the current 490 encoding mode, where $N$ is referred to as the prediction in491 terval. Let $P_{o}^{n+N}(m)$ denote the overflow probability at slot $492(n+N)$, which is defined as

$$
P_{o}^{n+N}(m)=P\left(Q_{n+N}^{m}>B_{h}\right)
$$

493 Subsequently, we derive a BOP estimation model based on the 494 aforementioned LDP [19], and then propose an online adaptive 495 source RC for video streaming over VBR channels.

\section{A. BOP Estimation}

497 When transmitting $D_{k}$ RLC SDUs containing video data in 498 slot $k$, the increased buffer length may be expressed as

$$
I_{k}=A_{k}-\min \left(Q_{(k-1)}, D_{k}\right) .
$$

499 Since we have $0 \leq A_{k} \leq A$ and $0 \leq D_{k} \leq D$, the set of values 500 for $I_{k}$ is $\{-D, \ldots, 0, \ldots, A\}$. Let us introduce the variable $501 \pi_{i}=P\left(I_{k}=i\right)$ to denote the probability of having a buffer 502 length increase of $I_{k}=i$. For a given wired network condition, $503 A_{k}$ is determined by the video encoding mode since the en504 coded video frame size is affected by the encoding mode, and $505 D_{k}$ is related to the channel bit rate. Their difference $I_{k}$ shows 506 the instantaneous mismatch between the video bit rate and the 507 channel bit rate. Due to the time-variant RLC SDU arrivals and 508 the fluctuating channel bit rate, the sequence $I_{i}(i=1,2, \ldots)$ 509 may frequently alternate between negative and positive values.
The total increase in the buffer length during the interval 510 spanning from slot $n$ to slot $(n+N)$ is given by

$$
I^{n+N}=\Sigma_{i=1}^{N} I_{n+i}
$$

Then, the buffer length $Q_{n+N}^{m}$ at the end of the slot $(n+N) 512$ may be expressed as

$$
Q_{n+N}^{m}=Q_{n}^{m}+I^{n+N}
$$

According to (6), the BOP at slot $(n+N)$ may be rewritten as 514

$$
P_{o}^{n+N}(m)=P\left(Q_{n}^{m}+I^{n+N}>B_{h}\right)
$$

Let us now define the expected value of the average buffer 515 length increase in each of the future $N$ slots as

$$
m_{o}=E\left[\frac{\sum_{i=1}^{N} I_{n+i}}{N}\right]
$$

where $E[\cdot]$ denotes the expectation operator.Furthermore, while 517 keeping the current encoding mode fixed as $m=M_{i}$, the 518 tolerable average buffer length increase during each slot is 519 defined as

$$
a_{o}=\frac{B_{h}-Q_{n}^{m}}{N}
$$

Having $m_{o} \geq a_{o}$ implies that there would be a high BOP after 521 $N$ time slots.

Let us now rewrite (19) as

$$
\begin{aligned}
P_{o}^{n+N}(m) & =P\left(Q_{n}^{m}+I^{n+N}>B_{h}\right) \\
& =P\left(I^{n+N} / N>\left(B_{h}-Q_{n}^{m}\right) / N\right) \\
& =P\left(\frac{\sum_{i=1}^{N} I_{n+i}}{N}>a_{o}\right) .
\end{aligned}
$$

The term $\sum_{i=1}^{N} I_{n+i} / N$ in (22) represents the average buffer 524 length change during a slot, which is jointly determined by the 525 video encoded bit rate controlled by the encoding mode and the 526 channel bit rate, whereas $a_{o}$ is the tolerable average increase in 527 the buffer length for each of the $N$ future slots, as determined by 528 the current buffer length. Therefore, the probability $P_{o}^{n+N}(m) 529$ in (22) may be viewed as an estimate of the remaining storage 530 capacity in the buffer, which may be used to smoothen the 531 fluctuation of the channel's affordable throughput in the future. 532 If the probability $P_{o}^{n+N}(m)$ exceeds a predefined threshold 533 value $p_{\text {qos }}$, we can decrease the encoding mode index to reduce 534 the video bit rate, thus decreasing both the BOP and the buffer- 535 induced delay.

Since $D_{k}(k=0,1, \ldots)$ are i.i.d. variables, $I_{k}(k=1,2, \ldots) 537$ are also i.i.d. random variables, which have a finite MGF of 538 $M(\theta)=E e^{\theta I_{k}}$ in the vicinity of 0 . Then, provided that $a_{o}>539$ $m_{o}$ is satisfied, according to (11), for large $N$, we have

$$
P_{o}^{n+N}(m) \approx \exp \left[-N L\left(a_{o}\right)\right]
$$


541 where

$$
\begin{gathered}
L\left(a_{o}\right)=\sup _{\theta>0}\left\{a_{o} \theta-\log M(\theta)\right\} \\
\log M(\theta)=\log \left\{\sum_{i=1-s_{D}}^{1} \pi_{i} \exp [i \theta]\right\} .
\end{gathered}
$$

542 To calculate the approximate BOP of (23), the knowledge 543 of $a_{o}, m_{o}$, and $\pi_{i}$ is required. It is straightforward to calculate $544 a_{o}$ according to (21). However, there is no prior knowledge 545 about the histogram of $I_{k}$, and hence, we cannot derive an546 alytical expressions for $m_{o}$ and $\pi_{i}$. Therefore, we have to 547 rely on their buffered history for estimating the current values 548 of these parameters using the classic sliding-window-based 549 method.

550 The observed buffer length increase/decrease sequence con551 stitutes the input of $\left\{I_{1}, I_{2}, I_{3}, \ldots\right\}$. The sliding window 552 takes into account the $N_{s}$ most recent $I_{k}$ values of $W_{n}=$ $553\left[I_{n}, I_{n-1}, \ldots, I_{n-N_{s}+1}\right]$.

554 For parameter $m_{o}$ of (20), we use the sample mean as its 555 estimate, i.e., we have

$$
\hat{m}_{o}=\frac{\sum_{i=n-N_{s}+1}^{n} I_{i}}{N_{s}} .
$$

556 Let $N_{i}(i \in\{-D, \ldots, 0, \ldots, A\})$ denote the number of 557 events when $I_{k}=i$ appears in the sliding window, which is 558 given by

$$
N_{i}=\sum_{k=n-N_{s}+1}^{n} 1\left(I_{k}=i\right)
$$

559 where $1(\cdot)$ is an indicator function. Then, the relative frequency 560 of encountering $I_{k}=i$ may be estimated as

$$
\hat{\pi}_{i}(n)=\frac{N_{i}}{N_{s}}
$$

561 which can be applied in (25) to estimate the BOP.

\section{B. Online Source RC Algorithm}

563 The RC strategy advocated will be discussed in the context of 564 three scenarios according to both the current buffer length $Q_{n}$ 565 and the average buffer length increase per slot $\hat{m}_{O}$ as follows.

566 1) $Q_{n}^{m} \geq B_{h}$ : This implies that the current buffer length is 567 above the threshold, which will impose an undesirable delay of 568 the video frame. Therefore, we should reduce the video bit rate 569 by decreasing the encoding mode index as

$$
m=M_{\max \{i-1,1\}} .
$$

570 2) $Q_{n}^{m}<B_{h}$ and $\hat{m}_{o} \geq a_{o}$ : In this scenario, although the 571 buffer length is under the threshold $B_{h}$, its average increase per 572 slot $\hat{m}_{O}$ exceeds the tolerable average increase $a_{o}$ of the buffer 573 length per slot during the forthcoming $N$ slots. This implies 574 that, at the current buffer length increase rate, the buffer length 575 will become higher than $B_{h}$ after $N$ slots. Therefore, in this case, the current video bit rate should be decreased to reduce 576 the BOP. Then, we can adjust the encoding mode according 577 to (29).

3) $Q_{n}^{m}<B_{h}$ and $\hat{m}_{o}<a_{o}$ : In this case, the current buffer 579 length is under the threshold $B_{h}$, and the average buffer length 580 increase per slot $\hat{m}_{o}$ is within the range defined by the capacity 581 $a_{o}$. Nevertheless, this does not imply that no buffer overflow 582 will occur in the forthcoming $N$ slots because $\hat{m}_{o}$ is the 583 average buffer length increase per slot, which cannot directly 584 characterize the buffer length increase in a certain time slot. 585 Hence, there is still a chance of buffer overflow. Fortunately, 586 since we have $a_{o}>\hat{m}_{o}$, buffer overflows remain a rare event. 587 According to the large deviation-based probability estimation 588 model of (23), for a sufficiently large $N$, the BOP may be 589 approximated as

$$
\hat{P}_{o}^{n+N}(m)=\exp \left[-N L\left(a_{o}\right)\right]
$$

An online measurement-based estimation method was pre- 591 sented in the previous section to calculate the BOP. Owing 592 to the exponential decay of the estimated BOP probability 593 with $N$, we can set $N$ to a moderate value for the sake of 594 acquiring an accurate BOP estimation instead of requiring a 595 large $N$.

Our proposed RC algorithm aims to adjust the encoding 597 mode to satisfy the BOP QoS requirement, i.e., $p_{\text {qos }}$. If we have 598 $\hat{P}_{o}^{n+N}(m) \geq p_{\text {qos }}$, this implies that the current encoding mode 599 index is too high to keep the BOP below $p_{\text {qos }}$. Therefore, we 600 should decrease the encoding mode index to reduce the video 601 bit rate, thus reducing the BOP. The future encoding mode 602 index is adjusted as $m=M_{\max \{i-1,1\}}$.

By contrast, if we have $\hat{P}_{o}^{n+N}(m)<p_{\text {qos }}$, the currently 604 affordable bit rate of the channel may be able to support a 605 higher video bit rate. Hence, we should increase the encoding 606 mode index to provide an improved video quality for the sake 607 of fully exploiting the attainable bit rate of the channel. To 608 achieve this, we define a threshold $p_{T}\left(<p_{\text {qos }}\right)$ for the BOP. 609 If $\hat{P}_{o}^{n+N}(m)<p_{T}$ is encountered consecutively $K>0$ times, 610 we will increase the encoding mode index according to

$$
m=M_{\min \{i+1, L\}} .
$$

The reason for requiring $K$ consecutive threshold viola- 612 tion occurrences to trigger an encoding mode index adjust- 613 ment is that this prevents frequent adjustments of the encod- 614 ing mode, which would result in perceivable video quality 615 fluctuations.

The RC regime is summarized in Algorithm 1. Although the 617 estimated average channel throughput was directly fed back to 618 the BTS to control the source rate in [18], this technique does 619 not characterize the burst-by-burst adaptive channel throughput 620 on a sufficiently fine timescale, which, hence, fails to guarantee 621 a low delay for the video packets. By contrast, Algorithm 1622 relies on the LDP of [19] to estimate the BOP, when the buffer 623 overflow is a rare event, and applies the BOP constraint to 624 trigger the source RC, thus achieving a low delay for video 625 packets. 


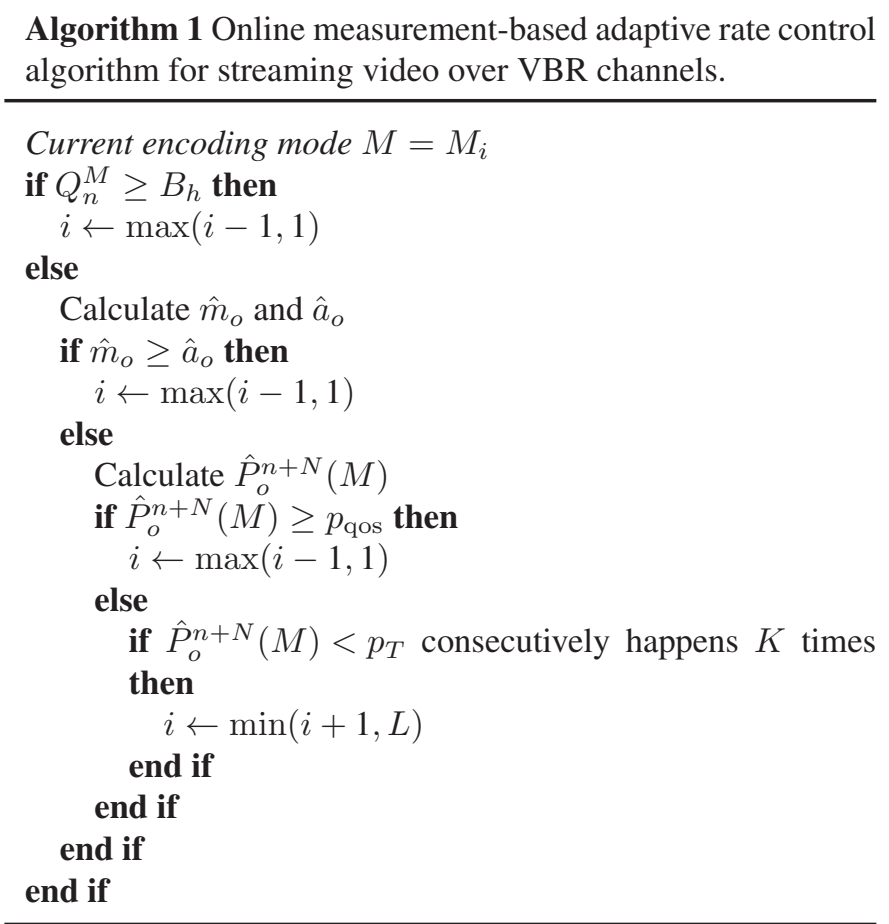

646 C. Discussion

647 Previously, the proposed solution was discussed in the con648 text of a single user requesting a single video stream. However, 649 it may be also extended to the scenario where multiple users 650 having a different link quality desire the same video. A simple 651 solution is for the video server to create a dedicated encoder 652 instance for each encoding mode, where multiple video streams 653 are generated by the encoders with the aid of different encoding 654 modes. Then, based on the feedback triggered by the proposed 655 method from the BTS, the video server may select an appropri656 ate video stream for each user associated with a different link 657 quality.

\section{Performance Evaluation}

659 Here, we characterize the performance of our online 660 measurement-based adaptive RC (MBARC) algorithm. We first 661 describe our simulation setup, including the network model 662 and the video sequences employed. Then, the metrics used for 663 performance evaluation are described. Finally, our simulation 664 results are presented and analyzed. In the simulations, we 665 also implemented the heuristic online adaptive RC (HOARC) 666 algorithm in [18] and an offline method to provide pertinent 667 performance comparisons to cutting-edge benchmarkers. The 668 offline method simply relied on all the encoding modes and 669 selected the mode having the best performance as the perfor670 mance benchmark.

\section{A. Simulation Setup}

672 We consider an HSPA network [1] relying both on AMC and 673 HARQ. We assume a UE (UE in HSPA parlance) belonging 674 to category 10. According to the HSPA specifications [2], the $675 \mathrm{CQI}$ value ranges from 0 to 30 , and the corresponding TB sizes
TABLE I

PROPERTIES OF THE VIDEO SEQUENCES

\begin{tabular}{|c|c|}
\hline Encoder & H.264 Full \\
\hline Resolution & $\begin{array}{l}\text { CIF }(352 \times 288): \\
\text { Silence of the Lambs } \\
\text { NBC } 12 \text { News } \\
\text { D-1(704 } \times 576) \text { : } \\
\text { The Shawshank Redemption }\end{array}$ \\
\hline Bit Rate & Variable Bit Rate (VBR) \\
\hline Number of Frames & 45,000 \\
\hline GoP Size & 16 \\
\hline Frames Rate & $30 \mathrm{fps}$ \\
\hline Video Duration & 25 minutes \\
\hline No. B frames between I/P frames & 1 \\
\hline
\end{tabular}

are $0,137, \ldots, 25558$ bits, respectively. In HSPA, there are 15676 time-division multiplex slots per 10-ms frame. Three 10/15 = 677 $2 / 3$ ms slots form a so-called transmission time interval (TTI) 678 of 2-ms duration, where only one user is allowed to transmit 679 with the aid of multiple spreading codes per TTI. Therefore, 680 the time-varying number of users may result in a time-varying 681 number of TTIs being assigned to each user, which, in turn, 682 leads to a time-varying throughput for each user. Therefore, we 683 simulated a multiuser scenario, where the maximum number 684 of concurrently communicating users was set to $U=8$, and 685 the new user arrival process follows a Markov process with an 686 arrival rate of $\lambda=10^{-4}$ per TTI. The service rate was assumed 687 to be $v=1.5 \times 10^{-5}$ per TTI. A round robin scheme was 688 applied to schedule the transmissions of the users. Then, our 689 proposed strategy is applied for one of the users to implement 690 its online source RC. Consider an i.i.d. Rayleigh channel for 691 the target user, where the received SNR $s$ is an exponentially 692 distributed random variable described by the probability density 693 function of $f(s)=(1 / \gamma) e^{-(s / \gamma)}$ having an average of $\gamma$. We 694 applied the SNR (in decibels)-to-CQI mapping in [40], i.e., 695

$$
\mathrm{CQI}=\lfloor\mathrm{SNR}+4.5\rfloor .
$$

According to the specifications, the CQI reporting cycle is 696 defined as 1, 2, 4, 5, 10, 20, 40, and 80 TTIs. In the simulations, 697 we set the CQI reporting cycle to four TTIs.

\section{B. Video Sequences Used for Performance Evaluations}

Three different video sequences are used in our simulations, 700 namely, the "Silence of the Lambs" clip, the "NBC 12 News" 701 clip [17], and the "Shawshank Redemption" clip, scanned at 30702 frames/s and encoded by the H.264 codec. The two former clips 703 have a Common Intermediate Format (CIF) resolution, whereas 704 the last clip has a D-1 resolution. The duration of the video 705 sequence is $25 \mathrm{~min}$, corresponding to 45000 video frames, 706 where a GOP is constituted by 16 frames. The properties of 707 these video sequences are listed in Table I. Here, the encoder 708 mode is denoted by $\left(m_{1}, m_{2}, m_{3}\right)$, where $m_{1}, m_{2}$, and $m_{3} 709$ represent the quantization scales for the I, P, and B frames, re- 710 spectively. The operational modes are listed as follows: $M 1=711$ $(10,10,12), \quad M 2=(16,16,18), \quad M 3=(22,22,24), \quad M 4=712$ $(24,24,26), \quad M 5=(28,28,30), \quad M 6=(34,34,36), \quad M 7=713$ 


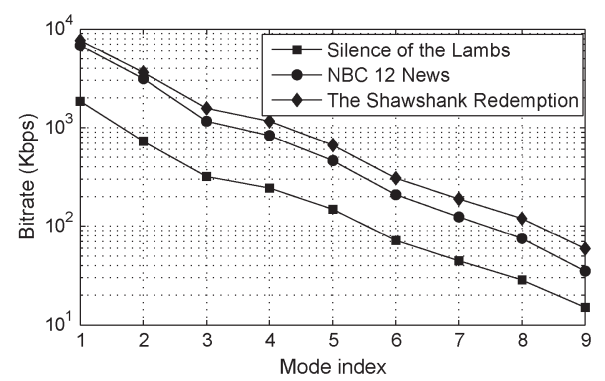

(a)

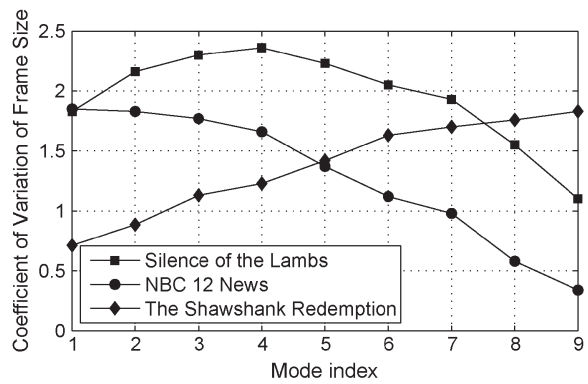

(b)

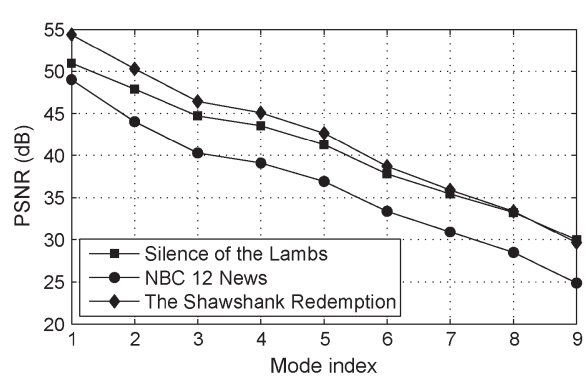

(c)

Fig. 6. Statistics of the video sequences. (a) Mean bit rate. (b) Coefficient of variation of frame size. (c) Mean PSNR.

$714(38,38,40), M 8=(42,42,44)$, and $M 9=(48,48,50)$. Fig. 6 715 characterizes the bit rate, the frame-size variation coefficient, 716 and the peak SNR (PSNR) statistics of the video sequences 717 in the different encoding modes, with the frame-size variation 718 coefficient being defined in [41] as follows:

$$
\operatorname{CoV}_{X}^{q}=\frac{1}{\bar{X}_{N}^{q}} \sqrt{\frac{1}{N-1} \sum_{n=0}^{N-1}\left(X_{n}^{q}-\bar{X}_{N}^{q}\right)^{2}}
$$

719 where $X_{n}^{q}$ denotes the frame size of video frame $n$ encoded 720 with the QP $q$, and $\bar{X}_{N}^{q}$ is the average frame size of the $N$ video 721 frames. In the first two simulations, the former two CIF clips 722 were used for basic performance investigations, whereas in the 723 rest of the simulations, the D-1 clip was used to characterize the 724 achievable performance in the case of higher resolution videos.

\section{C. Performance Metrics}

726 In the simulations, each RLC SDU carried the bits of video 727 frames. To satisfy the tolerable delay constraint of a specific 728 video application, the RLC SDUs are dropped from the buffer 729 when their delay exceeded the threshold of $l$ milliseconds. To 730 evaluate the quality of a received video sequence, an objective 731 video quality evaluation model similar to [43] is adopted, which 732 is defined by the rate of dropped video frames (DVFRs) that are 733 undecodable at the MS, i.e., by

$$
\mathrm{DVFR}=1-\frac{N_{\mathrm{dec}}}{N_{\text {total }-I}+N_{\text {total }-P}+N_{\text {total }-B}}
$$

734 where $N_{\text {dec }}$ is the total number of decodable frames, including 735 all three types of frames. The decoding dependence values 736 between different types of frames are also considered in count737 ing the decodable frames. A lower DVFR value implies that a 738 better quality is perceived by the recipient. Since the proposed 739 adaptation method uses different bit rates, when configured 740 for adapting the throughput and the quality, we also apply the 741 average PSNR as a further performance metric.

742 All the results were averaged over 100 independent sim743 ulation runs. To characterize the performance improvement 744 of our MBARC, we also conducted independent simulations 745 for each encoder mode, and the best results were selected as 746 performance benchmarks.

\section{Simulation Results}

1) Performance for Different Delay Thresholds: In the first 748 experiment, the parameters of the proposed online MBARC 750 algorithm were set as follows: length of the sliding window 751 $N_{s}=80$, prediction interval $N=80$, buffer length threshold 752 $B_{h}=16, p_{\mathrm{qos}}=10^{-5}, p_{T}=10^{-10}$, and $K=32$. The initial 753 mode index of the encoder is $M_{9}$, which has the lowest video 754 bit rate. The MBARC was activated every eight video frame 755 intervals. Its performance was investigated for the delay thresh- 756 olds of $\{60,100,140,180$, and $220 \mathrm{~ms}\}$, which cover the 757 delay requirements of various video applications. For example, 758 the delay limit of $60 \mathrm{~ms}$ is applicable to lip-synchronized 759 real-time interactive video conferencing applications, whereas 760 the delay limit of 100 or $140 \mathrm{~ms}$ is applicable to wireless 761 video surveillance, and finally, 180 and $220 \mathrm{~ms}$ are for digital 762 television broadcast or video-on-demand services. Fig. 7(a) and 763 (b) shows the DVFR and the average PSNR of Silence of the 764 Lambs and NBC 12 News for different delay thresholds at the 765 average channel SNR of $\gamma=20 \mathrm{~dB}$. It can be observed in Fig. 7766 that, as the encoder mode index increases, the DVFR decreases 767 owing to the reduced source rate. For Silence of the Lambs, 768 the DVFR of the operational mode $M 6$ is similar to the DVFR 769 of MBARC, but our MBARC improves the average PSNR by 770 about $3 \mathrm{~dB}$. For NBC 12 News, our MBARC and $M 7$ have 771 a similar DVFR, but MBARC improves the average PSNR by 772 about $1 \mathrm{~dB}$. This implies that the proposed MBARC is capable 773 of adaptively adjusting the encoder mode when the source rate 774 is temporarily higher than the affordable channel rate.

2) Performance for Different Channel SNRs: To investi- 776 gate the proposed MBARC's source rate adaptation capabil- 777 ity for different channel qualities, we conducted experiments 778 at different average channel SNRs, namely, at $\gamma=\{12,16,779$ $20,24$, and $28 \mathrm{~dB}\}$. The buffer length threshold was set to 780 28, whereas the remaining MBARC parameters were the same 781 as in the first experiment. We set the maximum delay, which 782 triggers dropping of the frames in the buffer to $200 \mathrm{~ms}$. For 783 each average channel SNR considered, we use an offline pro- 784 cedure to select the best encoding mode, which maximizes 785 the average PSNR, while maintaining a DVFR similar to that 786 of MBARC. The simulation results shown for the MBARC, 787 HOARC, and offline mode selection method were plotted in 788 Fig. 8(a) and (b). The results recorded in Fig. 8(b) for dif- 789 ferent average channel SNRs using the offline method were 790 marked with (Mode). Fig. 8(b) demonstrates the average PSNR 791 

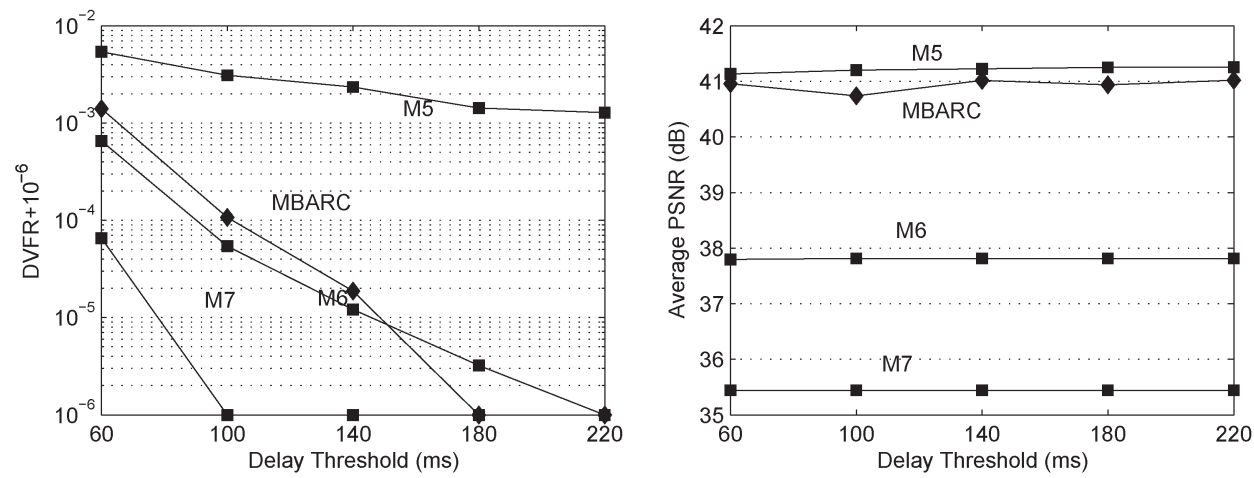

(a)
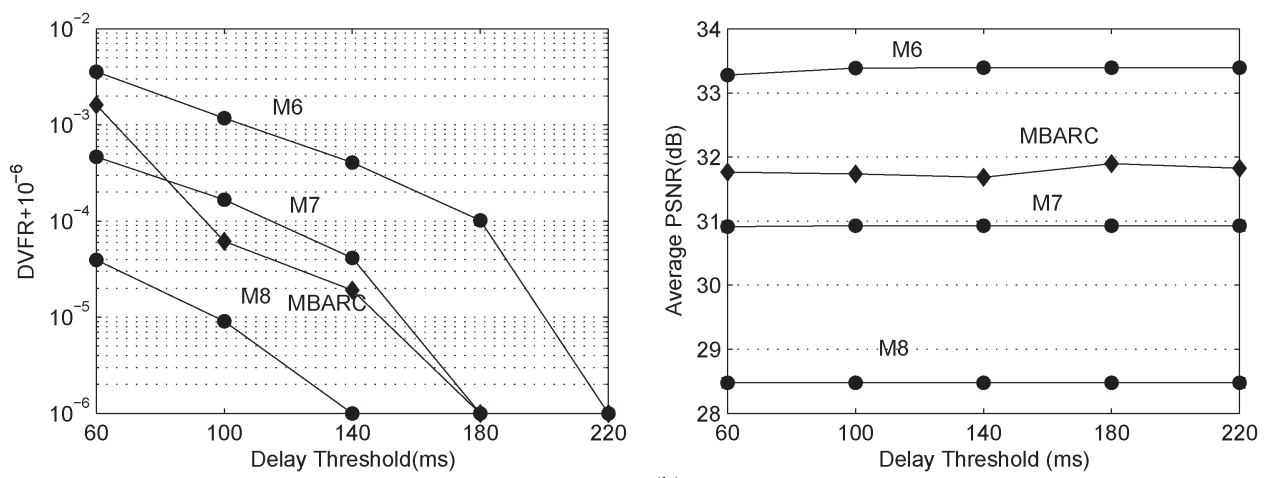

(b)

Fig. 7. (a) DVFR and Average PSNR of Silence of the Lambs for different delay thresholds with average channel SNR of $20 \mathrm{~dB}$. (b) DVFR and Average PSNR of NBC 12 News for different delay thresholds with average channel SNR of $20 \mathrm{~dB}$. (a) Silence of the Lambs. (b) NBC 12 News.

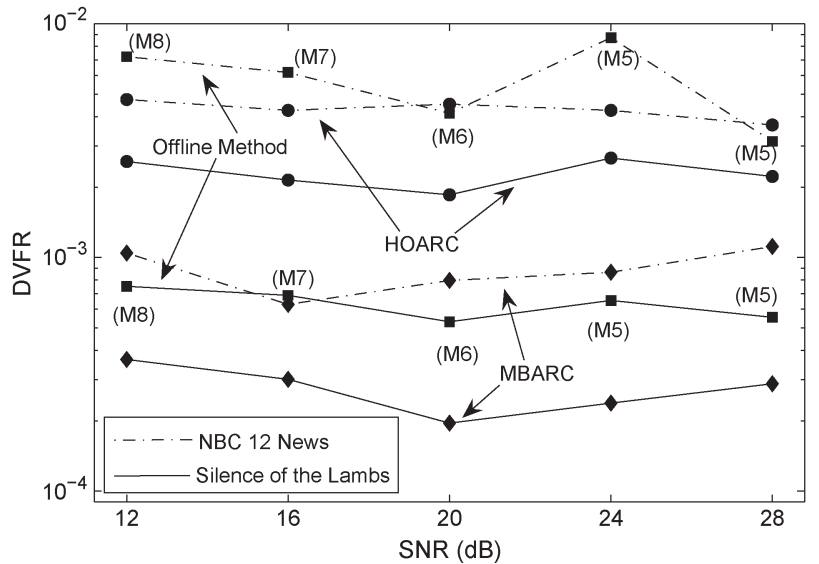

(a)

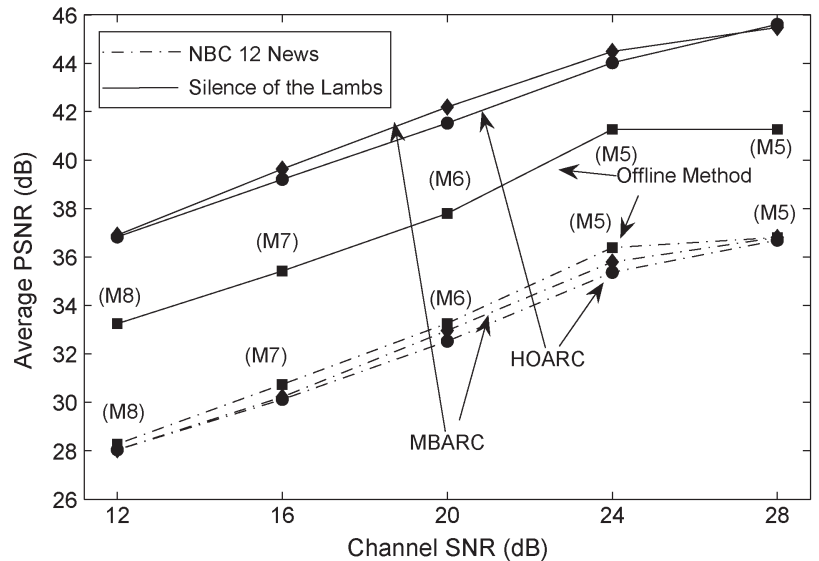

(b)

Fig. 8. (a) DVFR for different average channel SNRs of Rayleigh channel. (b) PSNR for different average channel SNRs of Rayleigh channel. The note in () represents the corresponding encoder mode for the offline method. (a) DVFR. (b) Average PSNR.

792 improvement of MBARC over the offline method, whereas its 793 DVFR remains lower than that of the offline method. This 794 demonstrates that MBARC is capable of adaptively accom795 modating different channel qualities by adjusting the encoding 796 mode without any prior knowledge of the channel quality. Al797 though Fig. 8(b) also shows that the HOARC technique in [18] 798 is capable of adapting to time-variant channel conditions and 799 approaching the PSNR performance of the proposed MBARC, 800 it suffers from a much higher DVFR than that of the MBARC 801 technique, as indicated in Fig. 8(a). The basic reason for this is elaborated as follows. The linear $\mathrm{AR}(1)$ of the HOARC 802 technique may not be appropriate for estimating the average 803 channel throughput since HSPA applies near-instantaneously 804 adaptive burst-by-burst transmissions. Furthermore, the aver- 805 age channel throughput cannot fully characterize the specific 806 near-instantaneously adaptive channel throughput at a certain 807 transmission time instant, which, in turn, increases the delay 808 of a specific packet and results in an increased probability of 809 delay constraint violation. By contrast, the proposed MBARC 810 technique is based on a BOP constraint, which may guarantee a 811 


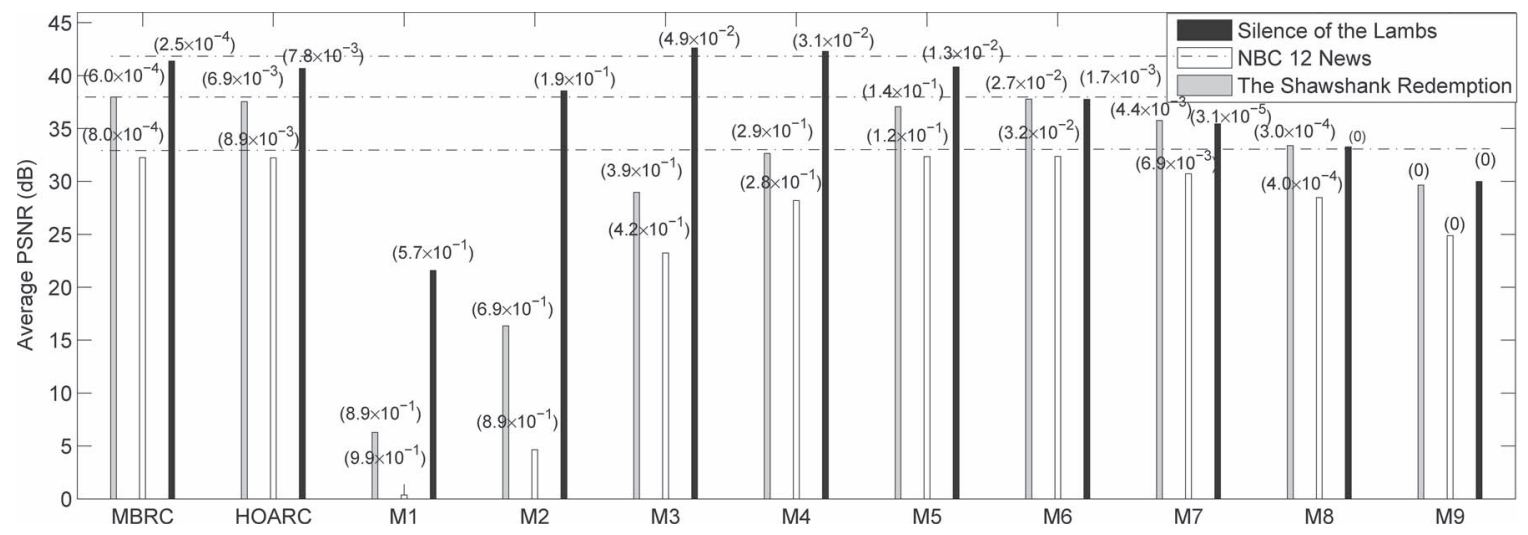

Fig. 9. Average PSNR for dynamic average channel SNRs for Rayleigh channel. The number in () represents the corresponding DVFR.

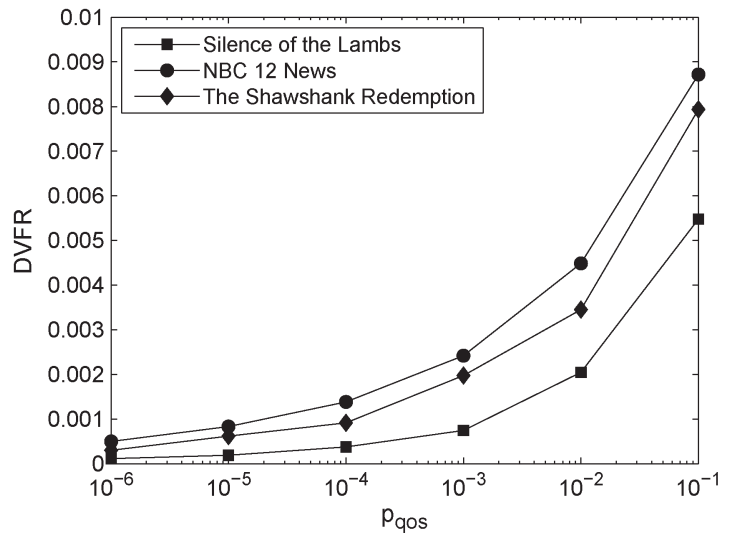

(a)

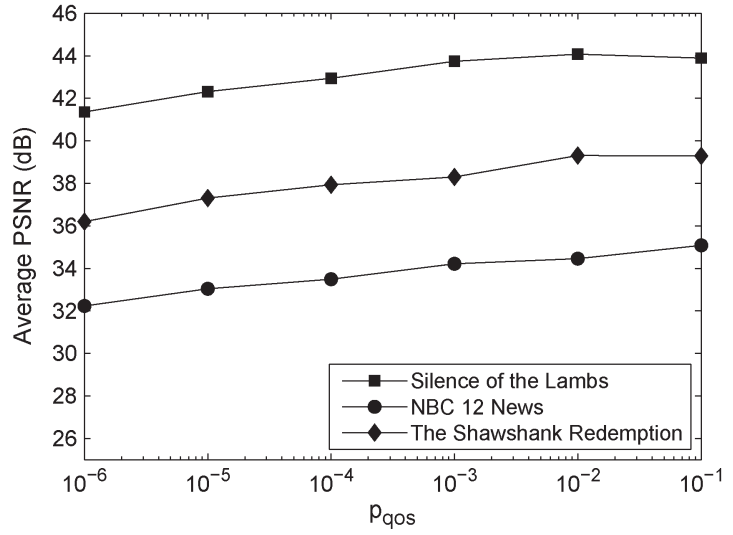

(b)

Fig. 10. (a) DVFR for different overflow probability thresholds $p_{\text {qos. }}$. (b) PSNR for different overflow probability thresholds $p_{\text {qos }}$. (a) Silence of the Lambs. (b) NBC 12 News.

812 low delay for the video packets. The application of the LDP [19] 813 assists MBARC in achieving an accurate estimation of BOP 814 and, thus, improves the DVFR performance.

815 3) Performance for Dynamic Channel Quality: The previous 816 investigations were conducted in a static environment by fixing 817 the average channel SNR. Next, we investigate the performance 818 of the MBARC in time-varying scenarios. The initial average 819 channel SNR was set to $24 \mathrm{~dB}$. At the instant of the 18000 th 820 video frame interval, the average channel SNR was suddenly 821 changed to $20 \mathrm{~dB}$. Then, at the instant of the 36000 th frame 822 interval, it was further reduced to $16 \mathrm{~dB}$. All the MBARC 823 parameters remained the same as in the previous experiment. 824 Fig. 9 shows the corresponding simulation results, where each 825 bar is marked with "(DVFR)" to characterize the corresponding 826 DVFR. It is shown that the DVFR of our MBARC is lower 827 than that of $M 6$ for Silence of the Lambs and that it improves 828 the PSNR by more than $2 \mathrm{~dB}$ in comparison with that of 829 M6. For NBC 12 News, although $M 8$ has a similar DVFR 830 to that of our MBARC, its average PSNR is $2 \mathrm{~dB}$ lower than 831 that of the proposed MBARC. This benefit is the result of 832 the MBARC's adaptability, which adjusts the encoder mode 833 online for VBR encoding to accommodate diverse dynamically 834 fluctuating propagation environments. By contrast, for a fixed 835 encoder mode, having a low channel SNR may inflict frame 836 dropping events, resulting in low video quality, whereas at high
SNRs, it fails to promptly decrease the video rate to improve 837 the video quality in a timely manner. Similarly, MBARC is 838 capable of adaptation and achieves an improved performance 839 for the Shawshank Redemption clip having a higher resolution. 840 Observe in Fig. 9 that the HOARC technique [18] approaches 841 the PSNR performance of the proposed MBARC, but it exhibits 842 a significantly higher DVFR than MBARC. This result also 843 illustrates the benefit of the explicit BOP constraint in the 844 problem formulation (4) and (5) and that of applying the LDP 845 for accurately estimating BOP.

4) Performance Sensitivity of the Proposed Algorithm: The 847 performance of the proposed method relies on parameters $N, 848$ $N_{s}$, and $K$, as shown in Algorithm 1. Hence, this section 849 investigates their effect on the performance of the proposed 850 method. In the related experiments, one of these three param- 851 eters was set to different values, whereas the other parameters 852 of our MBARC were the same as those in 2). The average SNR 853 value of our Rayleigh channel model was fixed to $20 \mathrm{~dB}$. The 854 remaining parameters of the channel model were set as in 2). $\quad 855$

Fig. 10 shows our simulation results of different BOP thresh- 856 olds $p_{\text {qos }}$ for the three clips. We may observe in Fig. 10 that 857 a reduced overflow probability threshold provides a reduced 858 DVFR. This is because a reduced probability threshold is 859 capable of activating timely adjustments of the encoder mode 860 to reduce the BOP. On the other hand, we can also observe in 861 


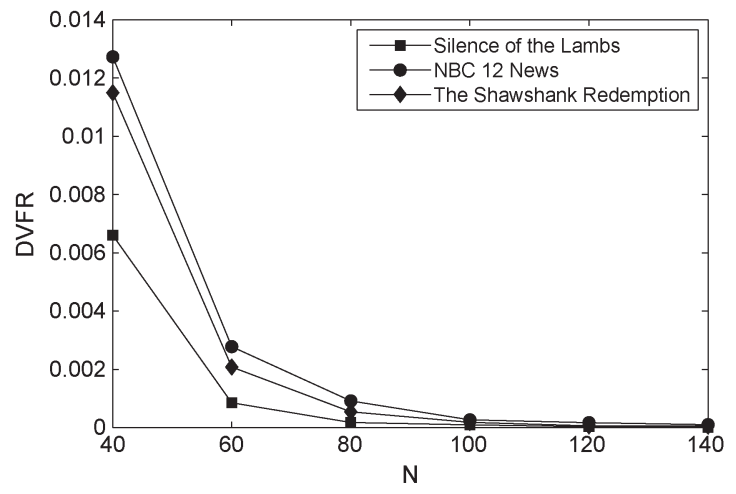

(a)

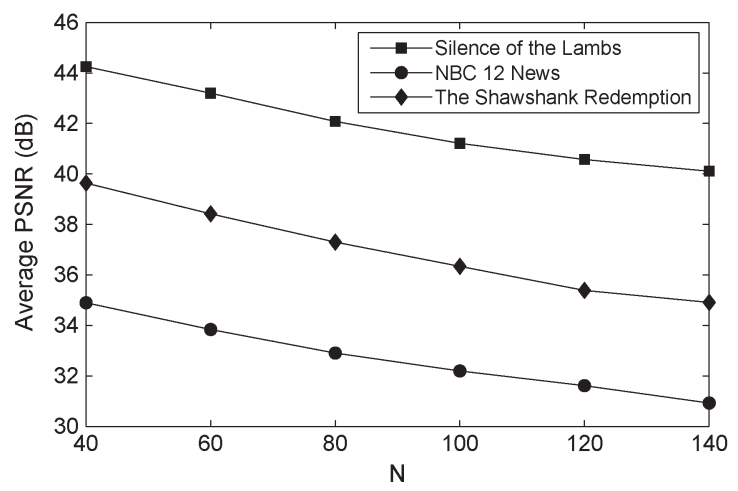

(b)

Fig. 11. (a) DVFR for different prediction intervals $N$. (b) PSNR for different predicting intervals $N$. (a) DVFR. (b) PSNR.

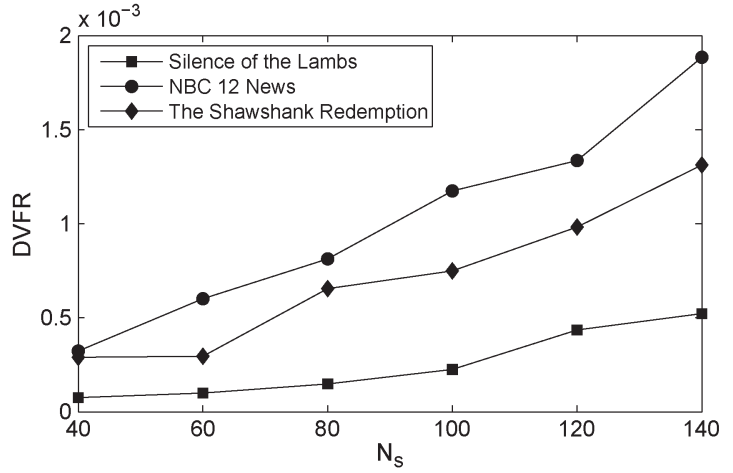

(a)

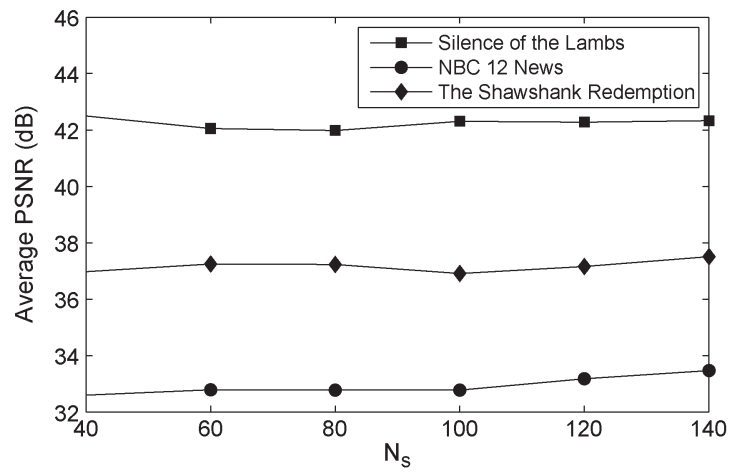

(b)

Fig. 12. (a) DVFR for different lengths of the sliding window $N_{s}$. (b) PSNR for different lengths of the sliding window $N_{s}$. (a) DVFR. (b) PSNR.

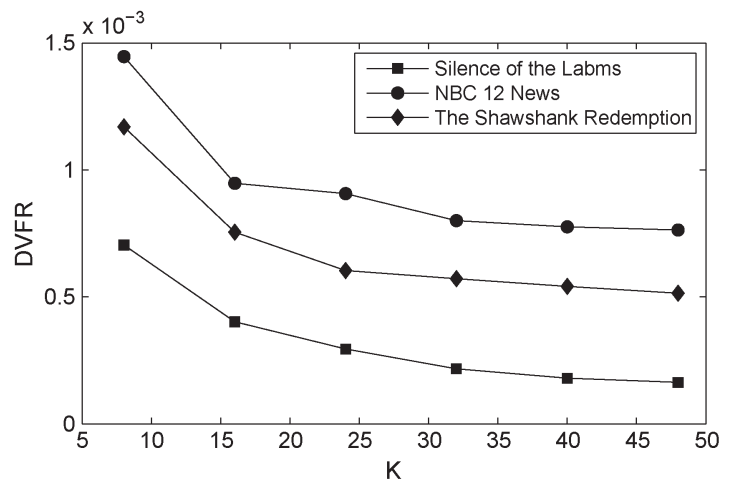

(a)

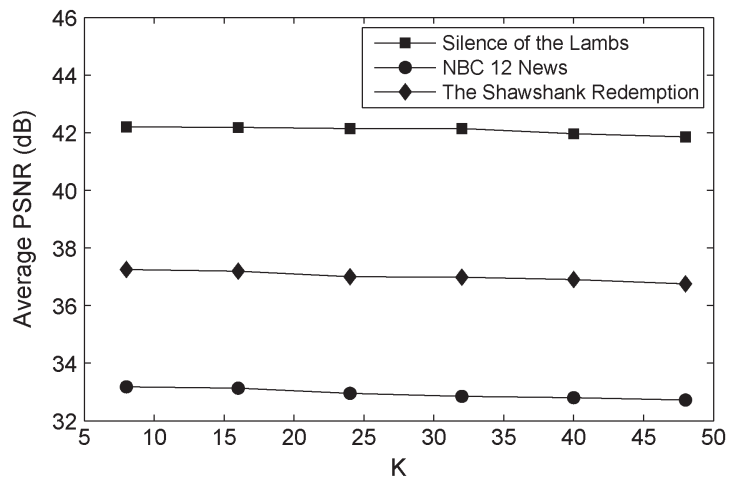

(b)

Fig. 13. (a) DVFR for different values of $K$. (b) PSNR for different values of $K$. (a) DVFR. (b) PSNR.

862 Fig. 10 that, as the BOP threshold decreases, the average PSNR 863 increases. This is caused by the lower encoding mode index 864 used to increase the video quality when the BOP threshold is 865 higher. It should be noted that, although the DVFR is related 866 to the BOP, their definitions are different. Hence, the value 867 of DVFR is not the same as $p_{\text {qos }}$, as shown in Fig. 10(a). 868 Fortunately, we may achieve an acceptable DVFR by setting $869 p_{\text {qos }}$ to an appropriate value such as $10^{-5}$.

870 Fig. 11 plots the simulation results for different prediction 871 intervals $N$ for the three clips. Fig. 11(a) shows that the DVFR 872 is rapidly reduced upon increasing $N$, whereas Fig. 11(b) shows 873 that the average PSNR is decreased as $N$ is increased. The basic reason is elaborated as follow. According to (30), a large 874 value of $N$ leads to a more accurate BOP estimate, which 875 assists the proposed method to trigger appropriate adjustments 876 of the encoding mode and thus to reduce the DVFR. The result 877 shows that $N \geq 80$ is appropriate for achieving an acceptable 878 performance for the proposed method.

Fig. 12 shows the performance sensitivity of the proposed 880 method to the sliding-window duration. It is shown in Fig. 12(a) 881 that a larger value of $N_{s}$ may result in a higher DVFR. This 882 may be caused by the reduced sensitivity of the buffer variance 883 when using a larger $N_{s}$ for estimating $\hat{m}_{o}$ according to (26). 884 Fig. 12(b) shows that $N_{s}$ has only a modest effect on the PSNR. 885 
886 Fig. 13 shows the sensitivity of the performance to parameter $887 K$. The proposed method triggers a video bit rate increase via 888 adjusting the encoding mode only when the threshold violation 889 of $\hat{P}_{o}^{n+N}<p_{T}$ is encountered consecutively $K$ times. There890 fore, having a larger $K$ implies a more conservative adjustment 891 policy, which leads to a lower DVFR and to a marginally 892 reduced PSNR, which are shown in Fig. 13(a) and (b).

\section{Conclusion}

894 An online adaptive source RC regime has been proposed for 895 streaming videos in HSPA and LTE-like VBR downlink sce896 narios. Large deviation theory was invoked to derive the online 897 measurement-based BOP model, applied at the RLC layer in the $898 \mathrm{BS}$. The advantage of the resultant algorithm is that it exploits 899 the online observations of buffer length and its variation for $900 \mathrm{RC}$, requiring no prior knowledge of the channel variations 901 and video characteristics. Our simulation results recorded in 902 multiuser scenarios demonstrated that the proposed algorithm 903 is capable of accommodating the channel quality variations, 904 which may be beneficial in HSPA and LTE-style environments.

905

906

907

908

909

910

911

912

913

914

915

916

917

918

919

920

921

922

923

924

925

926

927

928

929

930

931

932

933

934

935

936

937

938

939

940

941

942

943

944

945

946

947
948

948
949

\section{REFERENCES}

1] L. Hanzo, J. Blogh, and S. Ni, 3G, HSPA and FDD Versus TDD Networking: Smart Antennas and Adaptive Modulation. Piscataway, NJ, USA: Wiley/IEEE Press, 2008.

2] Third-Generation Partnership Project (3GPP), "Physical layer procedures (FDD)," ETSI, Sophia Antipolis, France, 3GPP TS25.214 V6.2.0, 2004.

3] M. Chen and A. Zakhor, "Rate control for streaming video over wireless," IEEE Wireless Commun., vol. 12, no. 4, pp. 32-41, Aug. 2005.

4] T. Chang and Y.-Q. Zhang, "A new rate control scheme using quadratic rate-distortion modeling," IEEE Trans. Circuits Syst. Video Technol., vol. 7, no. 1, pp. 246-250, Feb. 1997.

5] Z. G. Li et al., " A unified architecture for real-time video-coding systems," IEEE Trans. Circuits Syst. Video Technol., vol. 13, no. 6, pp. 472-487, Jun. 2003.

[6] Y. Liu, Z. G. Li, and Y. C. Soh, "Rate control of H.264/AVC scalable extension," IEEE Trans. Circuits Syst. Video Technol., vol. 18, no. 1, pp. 116-121, Jan. 2008.

7] "Joint Video Team Software JM 18.0." [Online]. Available: http://iphome.hhi.de/suehring/tml/

8] Z. Li et al., "Adaptive basic unit layer rate control for JVT," presented at the 7th Meeting, Pattaya II, Pattaya, Thailand, Mar. 2003, Paper JVTG012r1.

[9] L. Hanzo, P. Cherriman, and J. Streit, Video Compression and Communications. Hoboken, NJ, USA: Wiley, 2007.

0] Y. Huang, S. Mao, and S. F. Midkiff, "A control-theoretic approach to rate control for streaming videos," IEEE Trans. Multimedia, vol. 11, no. 6, pp. 1072-1081, Oct. 2009.

11] H. L. Lin, T. Y. Wu, and C. Y. Huang, "Cross layer adaptation with QoS guarantees for wireless scalable video streaming," IEEE Commun. Lett., vol. 16, no. 9, pp. 1349-1352, Sep. 2012.

12] N. Changuel, N. Mastronarde, M. Van der Shaar, B. Sayadi, and M. Kieffer, "Adaptive scalable layer filtering process for video scheduling over wireless networks based on MAC buffer management," in Proc. IEEE ICASSP, May 2011, pp. 2352-2355.

13] A. Dua, C. W. Chan, N. Bambos, and J. Apostolopoulos, "Channel, deadline, and distortion $\left(C D^{2}\right)$ aware scheduling for video streams over wireless," IEEE Trans. Wireless Commun., vol. 9, no. 3, pp. 1001-1011, Mar. 2010.

14] H. Zhang, Y. Zheng, M. A. Khojastepour, and S. Rangarajan, "Cross-layer optimization for streaming scalable video over fading wireless networks," IEEE J. Sel. Areas Commun., vol. 28, no. 3, pp. 344-353, Apr. 2010.

15] E. Piri, M. Uitto, J. Vehkapera, and T. Sutinen, "Dynamic cross-layer adaptation of scalable video in wireless networking," in Proc. IEEE GLOBECOM, Dec. 2010, pp. 1-5.
[16] R. Radhakrishnan and A. Nayak, "Cross layer design for efficient video 950 streaming over LTE using scalable video coding," in Proc. IEEE ICC, 951 Jun. 2012, pp. 6509-6513.

[17] Video Trace Library. [Online]. Available: http://trace.eas.asu.edu/ 953

[18] C. Chen, R. W. Heath Jr., A. C. Bovik, and G. D. Veciana, "A Markov 954 decision model for adaptive scheduling of stored scalable videos," IEEE 955 Trans. Circuit Syst. Video Technol., vol. 23, pp. 1081-1095, Jun. 2013. 956

[19] A. Dembo, and O. Zeitouni, Large Deviations Techniques and Applica- 957 tions. 2nd ed. Berlin, Germany: Springer-Verlag, 1998.

[20] Apple HLS, "HTTP Live Streaming draft-pantos-http-live-streaming-08 959 (IETF draft)."

960

[21] Third-Generation Partnership Project (3GPP), "Transparent end-to-end 961 Packet Switched Streaming Service (PSS); protocols and codecs," ETSI, 962 Sophia-Antipolis, France, 3GPP TS 26.234, 2010.

[22] C. Hsu, and M. Hefeeda, "Partitioning of multiple fine-grained scalable 964 video sequences concurrently streamed to heterogeneous clients," IEEE 965 Trans. Multimedia, vol. 10, no. 3, pp. 457-469, Apr. 2008.

[23] Z. Chen, M. Li, and Y. Tan, "Perception-aware multiple scalable video 967 streaming over WLANs," IEEE Signal Process. Lett., vol. 17, no. 7, 968 pp. 675-678, Jul. 2010.

[24] Third-Generation Partnership Project (3GPP), "Transparent end-to-end 970 Packet Switched Streaming Service (PSS); progressive download and 971 dynamic adaptive streaming over HTTP (3G)," ETSI, Sophia-Antipolis, 972 France, 3GPP TS 26.247, 2011.

[25] Information Technology-Dynamic Adaptive Streaming Over HTTP 974 (DASH)_Part 1: Media Presentation Description and Segment Formats, 975 ISO/IEC 23009-1:2012, 2012,

976

[26] G. Tian, and Y. Liu, "Towards agile and smooth video adaptation 977 in dynamic HTTP streaming," in Proc. ACM CoNEXT, Dec. 2012, 978 pp. 109-120.

[27] J. Jiang, V. Sekar, and H. Zhang, "Improving fairness, efficiency, and 980 stability in HTTP-based adaptive video streaming with FESTIVE," in 981 Proc. ACM CoNEXT, Dec. 2012, pp. 97-108.

[28] J. Chen, R. Mahindra, M. A. Khojastepour, S. Rangarajan, and 983 M. Chiang, "A scheduling framework for adaptive video delivery over 984 cellular networks," Proc. ACM MOBICOM, Sep. 2013, pp. 389-400. 985

[29] J. Little, "A proof of the queuing formula: $L=\lambda W$," Oper. Res., vol. 9, 986 no. 3, pp. 383-387, May 1961.

[30] A. Wilig, "A Short Introduction to Queueing Theory," 1999. [Online]. 988 Available: http://www.cs.ucf.edu/lboloni/Teaching/EEL6785_Fall2010/ 989 slides/QueueingTheory.pdf

[31] F. Ishizaki, and F. Takine, "Loss probability in a finite discrete-time queue 991 in terms of the steady state distribution of an infinite queue," Queue Syst., 992 vol. 31, no. 3/4, pp. 317-326, Jul. 1999.

[32] N. B. Shroff and M. Schwartz, "Improved loss calculations at an ATM 994 multiplexer," IEEE/ACM Trans. Netw., vol. 6, no. 4, pp. 411-421, 995 Aug. 1998.

996

[33] H. S. Kim and N. B. Shroff, "Loss probability calculations and asymp- 997 totic analysis for finite buffer multiplexers," IEEE/ACM Trans. Netw., 998 vol. 9 , no. 6, pp. 755-768, Dec. 2001.

[34] H. Bobarshad, M. van der Schaar, A. H. Aghvami, R. S. Dilmaghani, 1000 and M. R. Shikh-Bahaei, "Analytical modeling for delay-sensitive video 1001 over WLAN," IEEE Trans. Multimedia, vol. 14, no. 2, pp. 401-414, 1002 Apr. 2012.

[35] S. T. Cheng, M. H. Tao, and C. Y. Wang, "Adaptive channel switching for 1004 centralized MAC protocols in multihop wireless networks," IEEE Trans. 1005 Commun., vol. 58, no. 1, pp. 228-234, Jan. 2010.

[36] H. Bobarshad and M. Shikh-Bahaei, "M/M/1 queuing model for adap- 1007 tive cross-layer error protection in WLANs," in Proc. IEEE WCNC, 1008 Nov. 2009, pp. 1-6.

[37] Y. Xu et al., "Probabilistic analysis of buffer starvation in Markovian 1010 queues," in Proc. IEEE INFOCOM, Mar. 2012, pp. 1826-1834. 1011

[38] M. Mandjes, Large Deviations for Gaussian Queues: Modelling Commu- 1012 nications Networks. Hoboken, NJ, USA: Wiley, 2007.

[39] G. E. P. Box, G. M. Jenkins, and G. C. Reinsel, Time Series Analysis 1014 Forecasting and Control. Englewood Cliffs, NJ, USA: Prentice-Hall, 1015 1994.

1016

[40] Motorola and Nokia, "Revised CQI proposal," 3GPP RAN WG1, Sophia, 1017 Antipolis, France, Tech. Rep. R1-02-0675, Apr. 2002.

1018

[41] P. Seeling and M. Reisslein, "Video transport evaluation with H.264 1019 video traces," IEEE Commun. Surveys Tuts., vol. 14, no. 4, 4th Quart. 1020 2012.

[42] Gabin et al., "3GPP mobile multimedia streaming stands," IEEE Signal 1022 Process. Mag., vol. 27, no. 6, pp. 134-138, Nov. 2010.

[43] C. H. Lin, C. H. Ke, C. K. Shieh, and N. K. Chilamkurti, "The packet loss 1024 effect on MPEG video transmission in wireless networks," in Proc. IEEE 1025 AINA, Apr. 2006, pp. 565-572. 


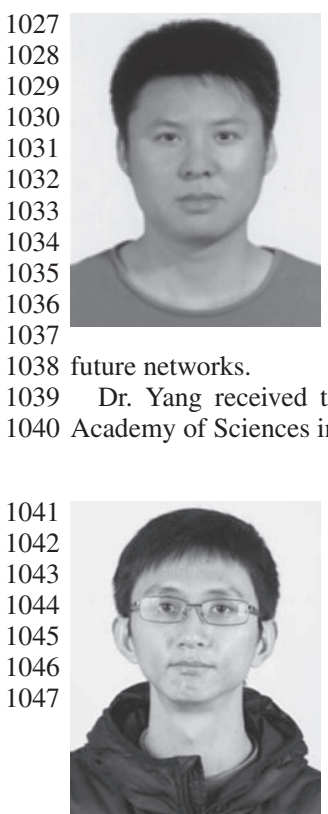

Jian Yang (M'08) received the B.S. and Ph.D. degrees from the University of Science and Technology of China (USTC), Hefei, China, in 2001 and 2006, respectively.

From 2006 to 2008, he was a Postdoctoral Scholar with the Department of Electronic Engineering and Information Science, USTC. Since 2008, he has been an Associate Professor with the Department of Automation, USTC. His current research interests include distributed system design, modeling and optimization, multimedia over wired and wireless, and

Lu Jia-Xi Young Talent Award from the Chinese (n)

Yongyi Ran received the B.S. and Ph.D. degrees in 2008 and 2014, respectively, from the University of Science and Technology of China, Hefei, China, where he is currently a Postdoctoral Researcher.

His research interests include cloud computing, service management, future networks, and stochastic optimization.

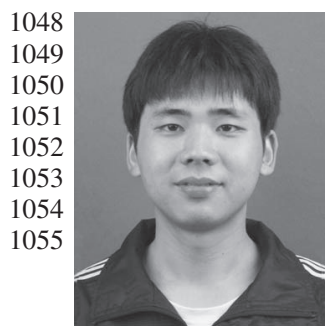

Shuangwu Chen received the B.S. degree in 2011 from the University of Science and Technology of China, Hefei, China, where he is currently working toward the Ph.D. degree with the School of Information Science and Technology.

His research interests include multimedia communications, future networks, and stochastic optimization.

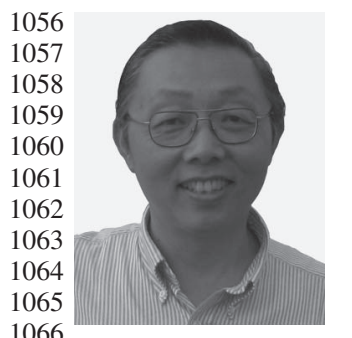

Weiping Li (F'00) received the B.S. degree from the University of Science and Technology of China (USTC), Hefei, China, in 1982 and the M.S. and $\mathrm{Ph} . D$. degrees from Stanford University, Stanford, CA, USA, in 1983 and 1988, respectively, all in electrical engineering.

In 1987, he joined the Faculty of Lehigh University, Bethlehem, PA, USA, as an Assistant Professor with the Department of Electrical Engineering and Computer Science. In 1993, he was promoted to Associate Professor with Tenure. In 1998, he was 1067 promoted to Full Professor. From 1998 to 2010, he was with several high1068 technology companies in the Silicon Valley, where he had technical and 1069 management responsibilities. In March 2010, he returned to the USTC and is 1070 currently a Professor with the School of Information Science and Technology. 1071 Prof. Li was elected Fellow of IEEE for contributions to image and video 1072 coding algorithms, standards, and implementations. He served as a member 1073 of Moving Picture Experts Group (MPEG) of the International Organization 1074 for Standardization (ISO) and an Editor of MPEG-4 International Standard. $1075 \mathrm{He}$ served as a Founding Member of the Board of Directors of the MPEG-4 1076 Industry Forum. His inventions on fine granularity scalable video coding and 1077 shape adaptive wavelet coding have been included in the MPEG-4 International 1078 Standard. As a Technical Adviser, he also made contributions to the Chinese 1079 Audio Video Coding Standard and its applications. He served as the Chair of 1080 several technical committees within the IEEE Circuits and Systems Society 1081 and at IEEE international conferences. He served as the Chair of the Best 1082 Student Paper Award Committee for the SPIE Visual Communications and 1083 Image Processing Conference. He served as the Editor-in-Chief of the IEEE 1084 TRANSACtions ON CiRCUITS AND SYSTEMS FOR Video TEChNOLOGY. 1085 He served as a Guest Editor for a special issue of the PROCEEDINGS OF THE 1086 IEEE. He has made many contributions to international standards. He received 1087 the Certificate of Appreciation from ISO and the International Electrotechnical 1088 Commission as a Project Editor in the development of International Standard 1089 in 2004, the Spira Award for Excellence in Teaching in 1992 from Lehigh 1090 University, and the first Guo Moruo Prize for Outstanding Student in 1980 from 1091 the USTC.

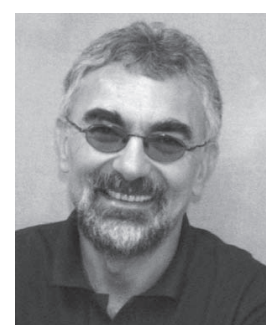

Lajos Hanzo (M'91-SM'92-F'04) received the 1092 Master's degree in electronics, the Ph.D. degree, and 1093 the Doctor Honoris Causa degree from the Technical 1094 University of Budapest, Budapest, Hungary, in 1976, 1095 1983, and 2009 respectively, and the D.Sc. degree 1096 from the University of Southampton, Southampton, 1097 U.K., in 2004.

1098

During his career in telecommunications, he 1099 has held various research and academic posts in 1100 Hungary, Germany, and the U.K. Since 1986, he has 1101 been with the School of Electronics and Computer 1102 Science, University of Southampton, Southampton, U.K., where he holds 1103 the Chair in telecommunications. He was a Chaired Professor of Tsinghua 1104 University, Beijing, China. He is the coauthor of 20 John Wiley/IEEE Press 1105 books on mobile radio communications, totalling in excess of 10000 pages, and 1106 has published more than 1400 research entries on IEEE Xplore. He is currently 1107 directing an academic research team, working on a range of research projects 1108 in the field of wireless multimedia communications sponsored by industry, 1109 the Engineering and Physical Sciences Research Council (EPSRC) U.K., the 1110 European IST Program, and the Mobile Virtual Centre of Excellence, U.K. He 1111 is an enthusiastic supporter of industrial and academic liaison, and he offers a 1112 wide range of industrial courses.

Dr. Hanzo has acted as a Technical Program Committee Chair for IEEE con- 1114 ferences, presented keynote lectures, and has received a number of distinctions. 1115 $\mathrm{He}$ is the Governor of the IEEE Vehicular Technology Society and the Past 1116 Editor-in-Chief of the IEEE Press. 


\section{AUTHOR QUERIES}

\section{AUTHOR PLEASE ANSWER ALL QUERIES}

AQ1 = Please check if "Star War IV" should be "Shawshank Redemption"

AQ2 = Please check if changes made in this sentence are appropriate.

AQ3 = Provided URL in Ref. [30] was not found. Please check.

\section{END OF ALL QUERIES}

